\author{
UNIVERSIDADE DE SÃO PAULO \\ INSTITUTO DE GEOCIÊNCIAS
}

\title{
SIMULAÇÃO GEOESTATÍSTICA ESPAÇOTEMPORAL COM DERIVA EXTERNA TEMPORAL LOCAL
}

\section{EDUARDO HENRIQUE DE MORAES TAKAFUJI}

Tese apresentada ao Programa Geociências (Recursos Minerais e Hidrogeologia) para obtenção do título de Doutor em Ciências

Área de concentração: Recursos Minerais

Orientador: Prof. Dr. Marcelo Monteiro da Rocha Coorientador: Prof. Dr. Rodrigo Lilla Manzione 
Autorizo a reprodução e divulgação total ou parcial deste trabalho, por qualquer meio convencional ou eletrônico, para fins de estudo e pesquisa, desde que citada a fonte.

Serviço de Biblioteca e Documentação do IGc/USP

Ficha catalográfica gerada automaticamente com dados fornecidos pelo(a) autor(a) via programa desenvolvido pela Seção Técnica de Informática do ICMC/USP

Bibliotecários responsáveis pela estrutura de catalogação da publicação: Sonia Regina Yole Guerra - CRB-8/4208 | Anderson de Santana - CRB-8/6658

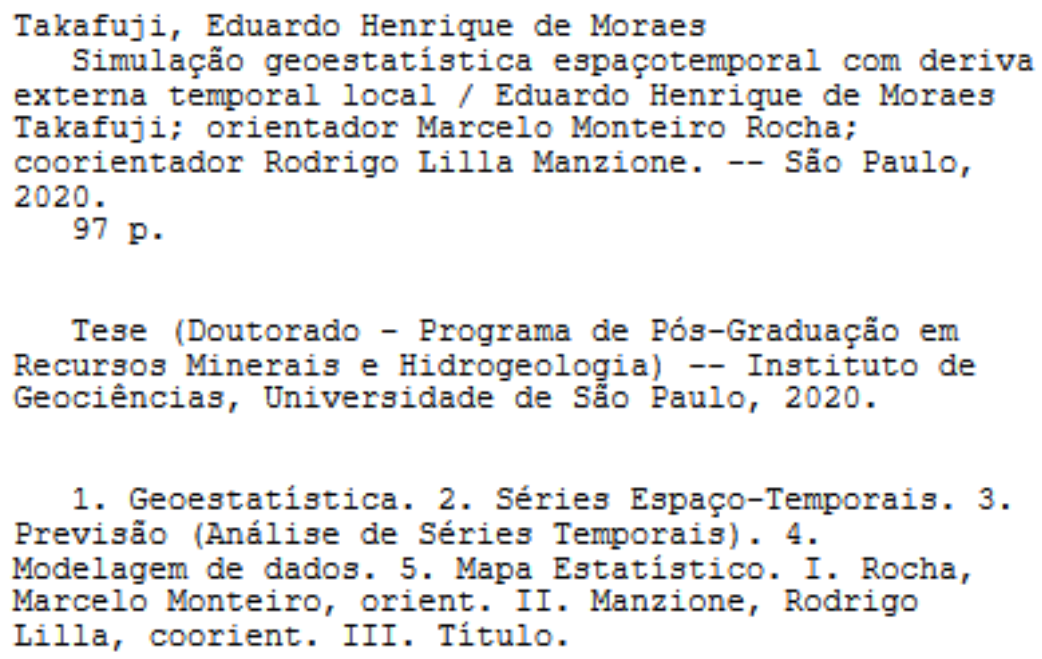




\section{UNIVERSIDADE DE SÃO PAULO INSTITUTO DE GEOCIÊNCIAS}

\section{SIMULAÇÃO GEOESTATÍSTICA ESPAÇOTEMPORAL COM DERIVA EXTERNA TEMPORAL LOCAL}

\section{EDUARDO HENRIQUE DE MORAES TAKAFUJI}

Orientador: Prof. Dr. Marcelo Monteiro da Rocha Coorientador: Prof. Dr. Rodrigo Lilla Manzione

Tese de doutorado

$N^{0} 617$

COMISSÃO JULGADORA

Dr. Marcelo Monteiro da Rocha

Dra. Michelle Chaves Kuroda Avansi

Dr. Claudio Benedito Baptista Leite

Dr. Jorge Kazuo Yamamoto

Dr. José Alberto Quintanilha

Dra. Cira Souza Pitombo 



\section{Agradecimentos}

O autor agradece a todos os envolvidos nas coletas, tratamento, armazenamento e distribuição dos dados. O dado MOD11C3 foi fornecido via internet por cortesia da NASA (Land Processes Distributed Active Archive Center - LP DAAC), USGS/Earth Resources Observarion and Science (EROS) Center, Sioux Falls, South Dakota, https://lpdaac.usgs.gov/data_access/data_pool. Dados das estações meteorológicas foram obtidos via internet pela National Oceanic and Atmospheric Administration (NOAA) - National Center for Environmental Information (NCEI). Além disso, o autor agradece a todos os envolvidos no projeto R, GADM e todos os colaboradores dos pacotes em R.

O autor agradece também a CAPES pela bolsa de doutoramento e a FAPESP (2016/09737-4) pelo apoio financeiro que contribuiu para 2 artigos relacionados a esta pesquisa de doutorado.

O autor é grato pelo apoio dos seus familiares (Eiji, Abigail, Paulo e Fernanda), seus amigos e colegas e especialmente ao pessoal da sala 105.

O autor reconhece com gratidão o trabalho duro de seu orientador Prof. Dr. Marcelo Monteiro da Rocha e seu coorientador Prof. Dr. Rodrigo Lilla Manzione. Foram muitas discussões, trabalhos e projetos para chegarmos aos resultados que gostaríamos.

Sem o trabalho duro e colaboração de todos estes profissionais, familiares e amigos, esta pesquisa não seria possível. Muito obrigado. 


\section{Epígrafe}

“... all models are approximations. Essentially, all models are wrong, but some are useful. However, the approximate nature of the model must always be borne in mind...."

Box and Draper 


\section{RESUMO}

Takafuji, E.H.M., 2020, Simulação Geoestatística Espaçotemporal com Deriva Externa Temporal Local [Tese de Doutorado], São Paulo, Instituto de Geociências, Universidade de São Paulo, 97p.

A geoestatística gera mapas ou modelos 3D estimados a partir de amostras pontuais distribuídas no espaço e, de maneira similar, a geoestatística espaçotemporal utiliza os mesmos princípios para dados obtidos no decorrer de uma época. Os métodos tradicionais de geoestatística espaçotemporal não consideram a variabilidade espacial das séries temporais. Isto ocorre em regiões grandes onde cada local seja possuidor de uma característica que altera a sua oscilação temporal. Para resolver este problema, propõe-se aqui um método de previsão de mapas mesclando a abordagem geoestatística com as decomposições de deriva externa temporais utilizados nas análises de séries temporais. Estas decomposições são necessárias para remover a tendência e sazonalidade dos dados estudados e, assim, garantir a estacionariedade de segunda ordem - necessária para a aplicação da geoestatística - na dimensão tempo. A decomposição de séries temporais é definida por alguns parâmetros como amplitude da oscilação sazonal, o coeficiente angular da reta, fase da oscilação e valor de intercepto. Estes parâmetros podem ser estimados no espaço para criar mapas de seus valores, sendo possível gerar modelos temporais determinísticos em cada ponto da área estudada. Paralelamente, os resíduos das decomposições (e.g. aleatoriedade, oscilações de diversas escalas, variabilidades espaçotemporais intrínsecas e outros) podem ser estimados ou simulados e, posteriormente, adicionados aos padrões espaçotemporais determinísticos. Os modelos temporais locais serão utilizados como deriva externa na geoestatística, permitindo conhecer seus resultados em qualquer local e data desejada, inclusive para o futuro (longo prazo). $\mathrm{O}$ estudo de caso para apresentar o método utiliza dados de temperatura da região da Patagônia, que foi selecionada, pois é uma região de mais de $1 \mathrm{mi} \mathrm{km}^{2}$ e apenas 18 estações meteorológicas. Foram selecionados os dados mensais de janeiro de 2002 a dezembro de 2016 para simular mapas de todos os meses do ano de 2017. Para validar os resultados, eles foram comparados com métodos propostos pela literatura (geoestatística espaçotemporal com e sem análise de tendências) e com os resultados de imagens de satélites (Land Surface Temperature). Os resultados mostram que o método proposto é mais robusto e preciso que os outros métodos encontrados na literatura, pois ele apresenta menor suavização e menor erro.

Palavras-chave: Geoestatística; Séries Espaço-Temporais; Previsão (Análise de Séries Temporais); Modelagem de dados; Mapa estatístico 


\begin{abstract}
Takafuji, E.H.M., 2020, Spatiotemporal Geostatistical Simulation with Local Temporal External Drift [Doctorate Thesis], São Paulo, Geoscience Institute University of São Paulo, $97 \mathrm{p}$.
\end{abstract}

Geostatistics generates estimated maps or 3D models from point samples distributed in space and, similarly, spatiotemporal geostatistics uses the same principles for data obtained over time. Traditional methods of spatiotemporal geostatistics do not consider the spatial variability of time series. This occurs in large regions where each location has a characteristic that changes the variation in time. To solve this problem, this work proposes a method of map prediction mixing the geostatistical approach with the external drift decompositions used in the analysis of time series. These decompositions are necessary to remove tendency and seasonality of the studied data and thus to guarantee the second order stationarity - necessary for the application of geostatistics - in the time dimension. Time series decomposition is defined by some parameters such as seasonal oscillation amplitude, the angular coefficient of the line, phase of oscillation, and intercept value. These parameters can be estimated in space to create maps of their values, and it is possible to create deterministic temporal models at each point of the studied area. Parallelly, the residuals (e.g. randomness, oscillations of several scales, intrinsic spatiotemporal variability, and others) can be estimated or simulated and added to the spatiotemporal deterministic patterns. Local time series models will be used as external drift in geostatistics, allowing to know their results at any desired date, including for the future (longterm). The case study presents the method and uses temperature data from the Patagonia region, which was selected, as it is a region of more than $1 \mathrm{mi} \mathrm{km}^{2}$ and only 18 weather stations. Monthly data from January 2002 to December 2016 were selected to simulate maps of every 2017 months. To validate the results, they were compared with methods proposed in the literature (spatiotemporal geostatistics with and without trend analysis) and satellite imagery (Land Surface Temperature) results. The results show that the proposed method is more robust and precise than the other methods found in the literature, as it has less smoothing and less error.

Key words: Geostatistics; Space-Time Series; Forecasting (Time Series Analysis); Data Modeling; Statistical Map 


\section{Sumário}

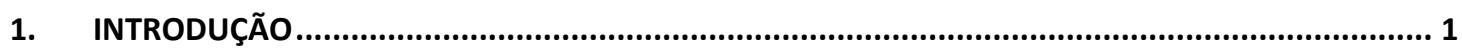

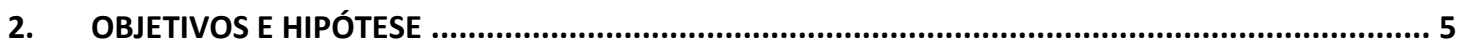

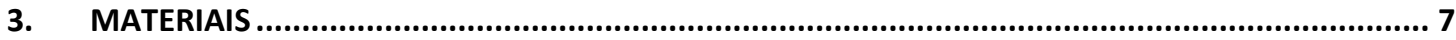

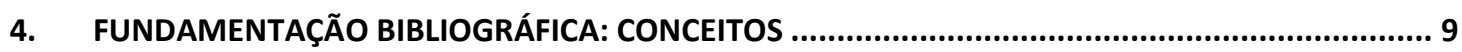

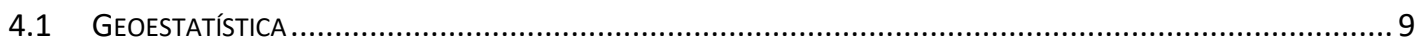

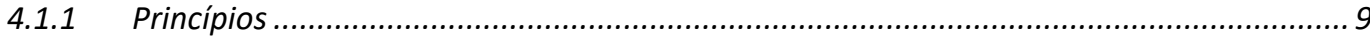

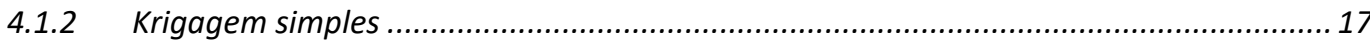

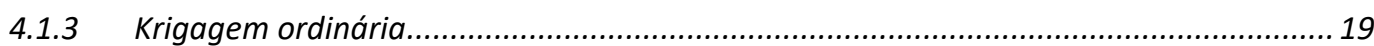

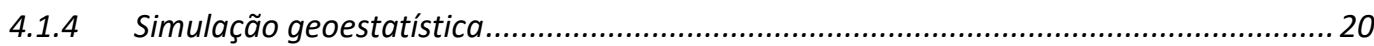

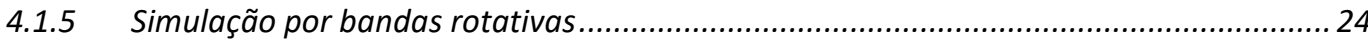

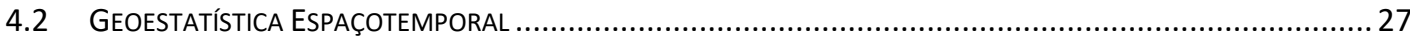

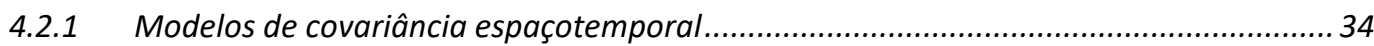

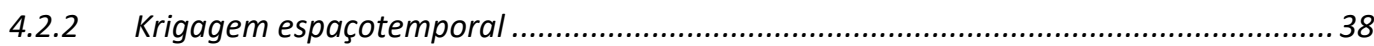

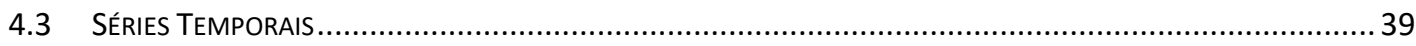

4.4 ReVISÃo MetodolóGICA: PreVISÃo ESPAÇOTEMPORAL ..................................................................... 47

5. MÉTODO DE SIMULAÇÃO ESPAÇOTEMPORAL GEOESTATÍSTICA COM DERIVA EXTERNA

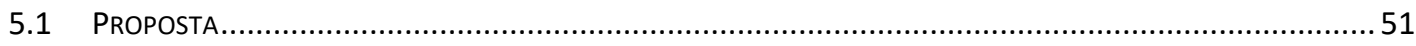

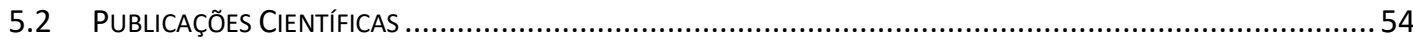

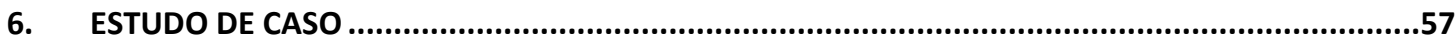

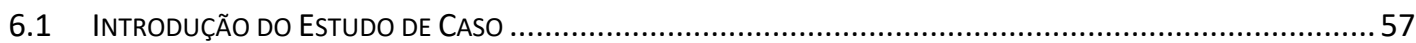

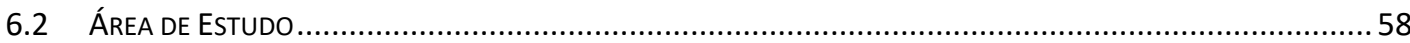

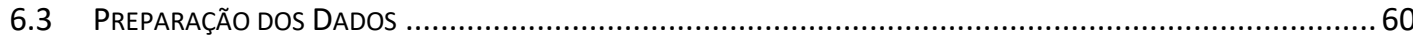

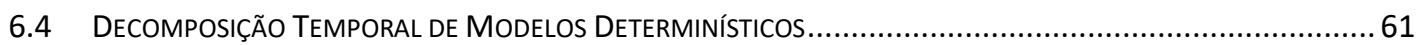

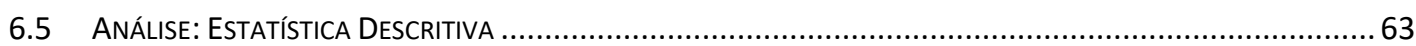

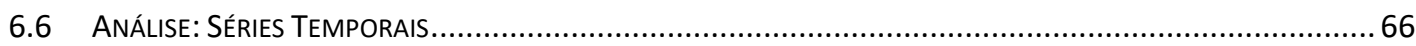

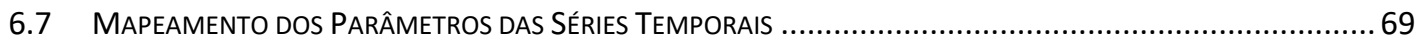

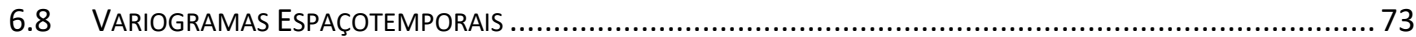

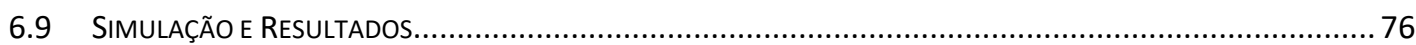

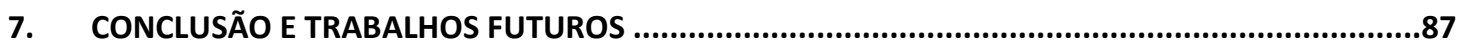

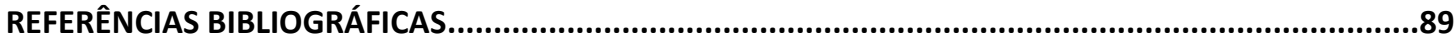




\section{Lista de Figuras}

Figura 1- Variograma típico (traduzida de Armstrong, 1998)

Figura 2-Comportamento próximo a origem: (i) quadrático; (ii) linear; (iii) descontinuidade; (iv) pepita puro (traduzida e modificada de Armstrong, 1998) 12

Figura 3 - Anisotropia Geométrica (a), Zonal (b), Mista (c) (Yamamoto, 2001a)............................................ 13

Figura 4 - Estrutura imbricada com duas amplitudes (traduzida e modificada de Armstrong, 1998)................. 13

Figura 5 - Variograma com efeito furo (traduzida e modificada de Deutsch, 2002) .......................................14

Figura 6 - Os principais modelos teóricos de semivariogramas (traduzida e modificada de Deutsch, 2002)........16

Figura 7 - Comparação entre os métodos de seleção de amostras. A figura A mostra a captura de dados com os pontos mais próximos; A figura B mostra a captura de dois dados para cada quadrante e a C mostra um dado para cada octante (modificada de Yamamoto, 2001b) ............................................................................. 17

Figura 8 - O princípio do método de bandas rotativas em 2D (Chilès e Delfiner, 1999) ....................................26

Figura 9 - Imagens resultantes de simulações com 1, 2 e 3 bandas comparadas com esquemas ilustrativos da contribuição de cada banda (Watanabe, 2003)

Figura 10- Em (A) o modelo conceitual para vetores de uma função aleatória Zi(s), $i=1, \ldots, T$ e em (B) a representação de vetores de uma série temporal $Z \alpha(t), \alpha=1, \ldots, n$ (traduzida e modificada de Kyriakidis e Journel, 1999)

Figura 11 - Cubo espaçotemporal. Amostras $S_{i}$ são regulares em $500 \times 500$ metros e foram coletadas em três momentos no tempo (Montero et al., 2015).

Figura 12 - Exemplo de covariância inteiramente simétrica (Montero et al., 2015)

Figura 13 - Representação esquemática da relação entre separabilidade, inteira assimetria, estacionariedade, $e$

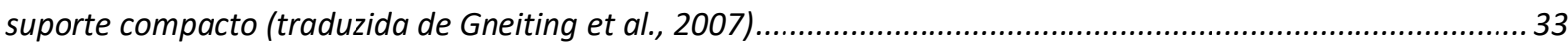

Figura 14 - Exemplo de pares de pontos separados por $h=1500 \mathrm{~m}$ e $u=2$ (Montero et al., 2015) ...................... 33

Figura 15 - Exemplo de variograma espaçotemporal (Montero et al. 2015) . .34

Figura 16 - Representação da distância espaçotemporal do modelo métrico (traduzida de Montero et al. 2015)

Figura 17 - Exemplo de uma função de autocorrelação (Cowpertwait e Metcalfe, 2009)

Figura 18 - Demonstração da modelagem de uma série temporal por sua tendência, sazonalidade e sua decomposição em variações aleatórias (traduzida de Cowpertwait e Metcalfe, 2009) 44

Figura 19 - Fluxograma mostrando os passos da simulação geoestatística com deriva temporal local

Figura 20 - Mapa da Patagônia e a localização das estações meteorológicas (azul) e vermelho estações que serão utilizadas de exemplo

Figura 21 - Séries temporais (estações $A$ e B) com a linha do ajuste seguido dos resultados decompostos em suas respectivas cores (vermelho para decomposição global e azul para local). 62

Figura 22 - Histograma e boxplot dos dados de temperatura (em Kelvin) das estações (calibração e controle) e imagens de satélite (LST) 
Figura 23 - Histograma e boxplot dos dados de temperatura decompostos globalmente e localmente

Figura 24 - Histograma e boxplot da raiz da média do quadrado do erro (RMSE) dos dados decompostos globalmente e localmente

Figura 25 - Séries temporais (estações $A$ e B) com a previsão por ARIMA dos dados brutos e decompostos (globalmente e localmente). A linha azul é o valor esperado, o campo cinza escuro é com incerteza de $80 \%$ e em cinza claro $95 \%$.

Figura 26 - Função autocorrelação (ACF) das estações $A$ e $B$ dos dados brutos e decompostos (globalmente e localmente). A linha azul é o intervalo de confiança de $95 \%$.

Figura 27 - Variograma experimental (pontos vermelhos) e modelo (linha preta) das variáveis regionalizadas amplitude e fase

Figura 28 - Variograma experimental (pontos vermelhos) das variáveis regionalizadas coeficiente angular e intercepto..

Figura 29 - Distribuição espacial da variável regionalizada amplitude e seu mapa krigado.....

Figura 30 - Distribuição espacial da variável regionalizada coeficiente angular e seu mapa interpolado ........... 71

Figura 31 - Distribuição espacial da variável regionalizada fase e seu mapa krigado...................................... 72

Figura 32 - Distribuição espacial da variável regionalizada intercepto e seu mapa interpolado ........................72

Figura 33 - Variograma espaçotemporal experimental e modelo teórico da variável temperatura brutos (sem decomposição).

Figura 34 - Variograma espaçotemporal experimental e modelo teórico da variável temperatura com decomposição global.

Figura 35 - Variograma espaçotemporal experimental e modelo teórico da variável temperatura com decomposição local..

Figura 36 - Diferença do variograma espaçotemporal experimental e modelo teórico da variável temperatura dos dados brutos e decompostos (globalmente e localmente)

Figura 37 - Mapa da média da simulação feita com os dados brutos e decompostos (globalmente e localmente) para a previsão do $12^{\circ}$ mês.

Figura 38 - Mapa da variância da simulação feita com os dados brutos e decompostos (globalmente e localmente) para a previsão do $12^{\circ}$ mês

Figura 39 - Mapa do P(5) da simulação feita com os dados brutos e decompostos (globalmente e localmente) para a previsão do $12^{\circ}$ mês.

Figura 40 - Mapa do P(95) simulação feita com os dados brutos e decompostos (globalmente e localmente) para a previsão do $12^{\circ}$ mês.

Figura 41 - Mapa do RMSE da simulação feita com os dados brutos e decompostos (globalmente e localmente)

Figura 42 - Distribuição acumulada dos dados da média de temperatura simulados com e sem decomposição (global e local) comparados com os dados das estações meteorológicas de calibração e imagem de satélite (LST) 
Figura 43 - Distribuição acumulada dos dados de $P(5)$ e $P(95)$ de temperatura simulados com e sem decomposição (global e local) comparados com os dados das estações meteorológicas de calibração e imagem de satélite (LST)

Figura 44 - Comparação mensal dos dados das estações meteorológicas de controle e imagem de satélite (LST) com as estatísticas (média, $P(5)$ e $P(95)$ dos valores de temperatura simulados com e sem decomposição (global e local)

Figura 45 - Comparação mensal do desvio padrão dos dados das estações meteorológicas de controle e imagem de satélite (LST) com o desvio padrão dos valores médios de temperatura simulados com e sem decomposição (global e local) 85

Figura 46 - Comparação mensal do RMSE dos dados das estações meteorológicas de controle e imagem de satélite (LST) com os valores do RMSE da média das simulações com e sem decomposição (global e local). 


\section{Lista de Tabelas}

Tabela 1 - Estatística dos dados de temperatura em Kelvin das estações (calibração e controle e imagens de satélite (LST)

Tabela 2 - Estatística descritiva dos dados de temperatura decompostos (global e local) das estações meteorológicas. 64

Tabela 3 - Parâmetros do ajuste do modelo teórico dos variogramas espaçotemporais

Tabela 4 - Estatística da média P(5) e P(95) da temperatura simulada (em Kelvin) com e sem decomposição (global e local) 77

Tabela 5 - Sumário das validações 86 


\section{INTRODUÇÃO}

Os estudos analíticos da variabilidade natural de variáveis regionalizadas foram desenvolvidos com o intuito de estimar valores não amostrados a partir da correlação espacial entre amostras em determinada região. Estas estimativas são, basicamente, métodos de interpolação que utilizam a dependência espacial (com ou sem anisotropia) para definir a relação de dependência espacial entre os dados amostrados e entre eles e os pontos a serem estimados. Estes métodos podem ser utilizados para estudos espaçotemporais, ou seja, de variáveis que, além de distribuição espacial, são coletados com certa frequência regular ou não, como, por exemplo, dados de estações meteorológicas e de poluição do ar, poços de controle de qualidade e nível do lençol freático, diversos tipos de informações de satélite e muitos outros. Variáveis espaçotemporais são coletadas, com maior frequência, com a melhoria das tecnologias de grandes dados, como informações coletadas por smartphones que têm localização espacial além de data e hora. Estas informações são de diversas naturezas como trânsito, saúde, crimes, temperatura, comportamento e muitas outras. Além disso, existem também informações espaçotemporais provenientes de satélites que monitoram diariamente diversos espectros que podem representar temperaturas, poluição, qualidade da vegetação, exposição do solo e outros. Existem outras variáveis espacializadas que têm uma componente temporal, como a definição do teor de corte de um minério em uma mina, a variação do preço de casas em uma cidade que são relacionadas a variáveis temporais monetárias.

Os cálculos de correlação espaçotemporais para estudos geoestatísticos seguem as mesmas regras da geoestatística espacial, portanto, os dados devem ser estacionários de segunda ordem e considerar a hipótese intrínseca tanto no espaço quanto no tempo. Qualitativamente, uma série estacionária atingiu o equilíbrio estatístico, ou seja, não existem tendências e remover os parâmetros de não-estacionariedade (tendências) é a maneira mais comum para garanti-la (Jenkins e Watts, 1968). Assim, se os dados apresentarem tendência e/ou sazonalidade temporais, estas podem ser modeladas deterministicamente e removidas para torná-los estacionários de segunda ordem e permitindo a previsão futura. Outra possibilidade é utilizar técnicas de diferenciação, como aplicado em alguns métodos de séries temporais, i.e., os modelos autoregressivos integrados com médias móveis (ARIMA), além disso, havendo volatilidade na variância em função do tempo, é possível utilizar técnicas de séries temporais heteroscedásticas como heteroscedasticidade condicional autoregressiva generalizada $(\mathrm{GARCH})$. 
Kyriakidis e Journel (1999) afirmam que os modelos de geoestatística espaçotemporal são utilizados para problemas ambientais, como monitoramento de deposição ácida, aquecimento global, previsão do tempo ou correntes de ventos. Cada área de estudo aborda o problema de modelar o espaço-tempo sob sua própria perspectiva, levando a diversos modelos semelhantes e, possivelmente, confusos. Os autores ainda comentam que estes modelos geoestatísticos espaçotemporais fornecem estrutura probabilística para o estudo dos dados e previsão que consideram a dependência da junção de observações no espaço e no tempo. Além disso, estes dados são tradicionalmente analisados por modelos desenvolvidos para distribuições ou no espaço ou no tempo e qualquer procedimento de agrupamento de ambas pode alterar a correlação espaçotemporal do fenômeno estudado, pois processos diferentes atuam em diferentes escalas no espaço e no tempo.

$\mathrm{O}$ aumento do número de atividades monitoradas e a necessidade de resolver problemas ambientais motiva o interesse em simular fenômenos naturais nas ciências ambientais, sendo que a maioria destes fenômenos podem ser observados como processos espaçotemporais (Nunes e Soares, 2005). Então, Porcu e Mateu (2007) comentam que modelos podem apresentar incertezas causadas por uma rede de observações incompleta, erros de medidas e localização, conhecimento incompleto das leis físicas que regem fenômenos ambientais e outros. Além disso, Costa e Soares (2009) destacam que fatores não climáticos podem alterar fatores climáticos e enviesar os resultados. Os principais fatores são relocação de estações, mudança de instrumentos de medida, alterações nas vizinhanças (como, por exemplo, construção de prédios e poda de árvores), imprecisão dos instrumentos e outros. Portanto, antes de qualquer estudo, é importante verificar possíveis irregularidades devido a estes fatores e validar o banco de dados.

Entre as diversas aplicações da geoestatística espaçotemporal, destacam-se estudos da natureza com diversas abordagens, como: mudança de suporte e krigagem bayesiana completa (Gelfand et al., 2001), análise multivariada (De Iaco et al., 2003 e Liu e Koike, 2007, De Iaco et al., 2013a), mapeamento de risco por simulações estocásticas (Soares e Pereira, 2007), aplicação de redes neurais (Russo e Soares, 2014), correção de viés e redução de escalas (Poggio e Gimona, 2015), krigagem ordinária incremental e recursiva (Zhong et al., 2016), Median Polish Kriging (Martínez et al., 2017) e krigagem espaçotemporal para previsão futura da profundidade relativa ao solo da água de um aquífero (Takafuji et al., 2018 e Manzione et al., 2019). Outras aplicações de análises espaçotemporais envolvem a abordagem hierárquica bayesiana de modelos espaçotemporais para estudar a difusão e previsão de leishmaniose 
(Assunção et al., 2001), topologia temporal (Nash et al. 2005), modelagem de fluxo de tráfego (Kamarianakis e Pastacos, 2005), teste para aplicações demográficas que mudam no espaçotempo (Schmertmann et al., 2010) e para aplicações de monitoramento de emergências por agrupamentos espaçotemporais de casos de doenças (Piroutek et al., 2014), observações de ratos em ambiente urbano (Tamayo-Uria et al., 2014), risco de roubo (Li et al., 2014), estudo de possível enviesamento das análises feitas para detectar e avaliar agrupamento (espaçotemporal) geográfico de riscos de doenças (Prates et al., 2014), interpolação de nível d'água, temperatura e condutividade elétrica no solo (Gasch et al., 2015) e a análise de padrões dos dados de vendas residenciais (Paci et al., 2017).

Os métodos baseados em dados e em estatísticas, como séries temporais e geoestatística, podem ser utilizados para mapas de previsões. Porém regiões muito grandes que apresentam alta variabilidade espacial das suas séries temporais tem resultados enviesados se sua variabilidade local não for considerada. Para resolver estes problemas, propõe-se a simulação espaçotemporal geoestatística com deriva externa temporal local. Este método utiliza modelos determinísticos que removam a tendência e sazonalidade da variável, em cada posição espacial amostrada, geram mapas destes parâmetros a fim de realizar a simulação geoestatística em dados estacionários de segunda ordem. Posteriormente, ele devolve estas variações em cada ponto da malha simulada. A simulação espaçotemporal pode extrapolar os limites no tempo e gerar mapas simulados futuros e os modelos das regressões das séries temporais locais também podem ser calculados para datas futuras. Este método apresenta resultados que respeitam a correlação espacial e com valores mais precisos e acurados que os métodos encontrados na bibliografia (sem decomposição e com deriva temporal global).

Gerar mapas de como será a distribuição de uma variável e sua incerteza no futuro é essencial para tomada de decisões no presente. Por exemplo, pode-se estimar as regiões e datas de maior probabilidade de incêndios florestais, fazer estudo de movimentação de poluentes, tendências de temperaturas e chuvas, entre outros. Então, a simulação espaçotemporal é fundamental para diversas áreas da ciência. Esta ferramenta cria modelos equiprováveis, desta forma é possível analisar o melhor e o pior cenários, probabilidade de ocorrência de desastres (valores acima ou abaixo de limiares aceitáveis), incertezas associadas aos mapas e, além disso, com as estatísticas descritivas e espaciais preservadas. 


\section{OBJETIVOS E HIPÓTESE}

O objetivo deste estudo é propor a integração da geoestatística espaçotemporal com as decomposições das análises de séries temporais e, assim, fazer a simulação de previsão de eventos regionais com o mapeamento local de tendências e sazonalidades. O método proposto é chamado de simulação espaçotemporal com deriva externa temporal local.

A hipótese principal é que o método proposto é robusto e adequado para mapear variáveis que apresentam variabilidade espacial das oscilações temporais e fazer previsões de longo prazo sem enviesar as estatísticas espaçotemporais. Assim, espera-se que os resultados reproduzam as estatísticas descritivas e que o erro local seja baixo mesmo em longos períodos futuros. Esta proposta é acompanhada de um procedimento para guiar a implementação desta simulação.

O objetivo secundário é realizar o estudo em uma situação extrema para analisar a robustez do método. A fim de testar e validar o método, ele foi aplicado aos dados obtidos por estações meteorológicas na Patagônia e validadas com outros métodos (Simulação espaçotemporal dos dados brutos e dos dados decompostos globalmente) e imagens de satélite (Land Surface Temperature - LST). Esta é uma região crítica, pois existe pouca informação para uma área muito grande e com alta variabilidade climática, ou seja, alta variabilidade espacial das séries temporais. 


\section{MATERIAIS}

Este trabalho foi desenvolvido no Laboratório de Informática Geológica da Universidade de São Paulo (LIG-USP) utilizando duas bases de dados com medidas de temperatura da região da Patagônia: a primeira coletada em estações meteorológicas (Global Summary of The Day - GSOD, 2018) que foi fornecida via internet pela National Oceanic and Atmospheric Administration (NOAA) - National Center for Environmental Information (NCEI) através do portal Climate Data Online (CDO); e a segunda base de dados é um produto de imagens de satélites (Land Surface Temperature - Wan et al., 2015) fornecida via internet por cortesia da NASA (Land Processes Distributed Active Archive Center - LP DAAC), USGS/Earth Resources Observarion and Science (EROS) Center, Sioux Falls, South Dakota, https://lpdaac.usgs.gov/data_access/data_pool.

Os bancos de dados de estações meteorológicas da Patagônia utilizados são dados mensais de temperatura medidos ao longo de 15 anos (janeiro de 2002 a dezembro de 2016) para simulá-las mês a mês em 2017. O local de estudo foi escolhido por ser uma grande área (mais de $1 \mathrm{mi} \mathrm{km}^{2}$ ) onde existem apenas 18 estações meteorológicas e o clima da região tem grande variabilidade, principalmente na região da cordilheira dos Andes.

O processamento foi feito na linguagem de programação R (R Core Team, 2017) e utilizou os seguintes pacotes: 'covatest' (De Iaco et al., 2018); 'forecast' (Hyndman e Khandakar, 2008; Hyndman et al., 2019); 'geoR' (Ribeiro Jr e Diggle, 2018); 'gdalUtils'(Greenberg e Mattiuzzi, 2018); 'gstat' (Pebesma, 2004; Gräler et al., 2016; Pebesma e Graeler, 2019); 'maptools' (Bivand e Lewin-Koh, 2019); 'raster' (Hijmans, 2019); 'spacetime' (Pebesma, 2012; Bivand et al., 2013; Pebesma et al., 2018); 'zoo' (Zeileis e Grothendieck, 2005; Zeileis et al., 2019). 


\section{FUNDAMENTAÇÃO BIBLIOGRÁFICA: CONCEITOS}

\subsection{Geoestatística}

\subsubsection{Princípios}

Clark (1980) discorre que normalmente os pontos mais próximos têm maior relação que pontos distantes. Em termos geoestatísticos, se pontos são muito relacionados, a variância espacial vai ser baixa. Então, pode-se considerá-la como uma medida da influência das amostras sobre áreas vizinhas. Landim (1997) discorre que a geoestatística é parte especial da estatística aplicada que trata de problemas referentes às variáveis regionalizadas, as quais apresentam uma continuidade no espaço e podem ser representadas por funções numéricas que assumem um valor definido a cada ponto no espaço descrevendo um fenômeno natural. Matheron (1963) definiu que uma variável regionalizada é, sensu stricto, uma função com valor definido em cada ponto do espaço, então do ponto de vista físico ou geológico, o autor descreve as características qualitativas relacionadas às variáveis regionalizadas, como:

(a) Localização: suas variações ocorrem no volume do depósito e é chamada de campo geométrico da regionalização;

(b) Suporte geométrico: formato geométrico, tamanho e orientação das amostras;

(c) Continuidade: expressa através de um desvio maior ou menor entre os valores de amostras vizinhas;

(d) Anisotropia: existência de uma direção preferencial em que as amostras não variam significativamente, enquanto em outra elas variam rapidamente.

Matheron (1971) salienta que a geoestatística é a aplicação da teoria das variáveis regionalizadas para estimar depósitos minerais ou, de maneira geral, quanto um fenômeno se espalha no espaço e exibe certa estrutura espacial. A teoria das variáveis regionalizadas tem dois propósitos principais, do lado teórico é expressar as propriedades estruturais de forma adequada e do lado prático é resolver os problemas de estimativas de variáveis regionalizadas em amostragens fragmentadas. Ambos propósitos são relacionados a uma região amostrada e o erro da estimativa depende das características estruturais, por exemplo: ele é maior quando as variáveis regionalizadas são irregulares e com mais descontinuidades nas variações espaciais.

Matheron (1971) discorre que se deve poder reconstruir, pelo menos em partes, a lei das funções aleatórias Z(s), onde valores z(s) são baseados para serem uma realização implicando que a inferência estatística é possível. Em geral, a inferência estatística não é possível se apenas 
uma realização $\mathrm{Z}(\mathrm{s})$ for obtida de $\mathrm{Z}(\mathrm{s})$. Para tornar a inferência estatística possível, deve-se introduzir hipóteses suplementares sobre a função aleatória $\mathrm{Z}(\mathrm{s})$ para reduzir os parâmetros aos quais esta lei é vinculada. Por exemplo, a hipótese da estacionariedade onde uma função aleatória estacionária, de certo modo, se repete no espaço e esta repetição dá novas oportunidades para inferências estatísticas.

Algumas definições de características essenciais para a aplicação da geoestatística foram definidas e descritas em detalhes por Matheron (1971) conforme:

(a) Função aleatória estacionária (Matheron, 1971): Z(s) é chamada de estacionária quando sua lei é invariante sob translação, em outras palavras, sejam s s, s2, .., $\mathrm{s}_{\mathrm{k}} \mathrm{k}$ pontos arbitrários, a variável aleatória $\mathrm{Z}\left(\mathrm{s}_{1}\right), \mathrm{Z}\left(\mathrm{s}_{2}\right), \ldots, \mathrm{Z}\left(\mathrm{s}_{\mathrm{k}}\right)$, dado qualquer vetor $\mathrm{h}$, tem a mesma distribuição (de k variáveis) que a variável aleatória $\mathrm{Z}\left(\mathrm{s}_{1}+\mathrm{h}\right), \mathrm{Z}\left(\mathrm{s}_{2}+\mathrm{h}\right)$, ..., $\mathrm{Z}\left(\mathrm{s}_{\mathrm{k}}+\mathrm{h}\right)$. Desta forma, $\mathrm{Z}(\mathrm{s})$ representa uma função aleatória estacionária.

(b) Esperança (Matheron, 1971): considera-se um ponto $s_{0}$, se $Z\left(s_{0}\right)$ tem esperança matemática $\left(E\left[Z\left(s_{0}\right)\right]\right)$, então a função $\mathrm{m}\left(\mathrm{s}_{0}\right)=\mathrm{E}\left[\mathrm{Z}\left(\mathrm{s}_{0}\right)\right]$, mas se $\mathrm{Z}(\mathrm{s})$ é estacionária $\mathrm{e}$, consequentemente, $\mathrm{m}\left(\mathrm{s}_{0}+\mathrm{h}\right)=\mathrm{m}\left(\mathrm{s}_{0}\right)$ para qualquer vetor $\mathrm{h}$, então $\mathrm{m}\left(\mathrm{s}_{0}\right)$ é uma constante $\mathrm{m}$ independente de $\mathrm{s}_{0}: \mathrm{m}=\mathrm{E}[\mathrm{Z}(\mathrm{s})]$

(c) Covariância C(h) (Matheron, 1971): considera-se dois pontos $\mathrm{s}_{0} \mathrm{e} \mathrm{s}_{0}+\mathrm{h}$, se as duas variáveis aleatórias $\mathrm{Z}\left(\mathrm{s}_{0}\right)$ e $\mathrm{Z}\left(\mathrm{s}_{0}+\mathrm{h}\right)$ têm variâncias finitas, eles apresentam covariância $\mathrm{C}\left(\mathrm{s}_{0} ; \mathrm{h}\right)$ que, a princípio, depende do ponto s 0 e do vetor h. Mas, como $\mathrm{Z}(\mathrm{s})$ é estacionária: $\mathrm{C}\left(\mathrm{s}_{0}+\mathrm{a} ; \mathrm{h}\right)=\mathrm{C}\left(\mathrm{s}_{0} ; \mathrm{h}\right)$ para qualquer vetor $\mathrm{h}$. Então, $\mathrm{C}\left(\mathrm{s}_{0} ; \mathrm{h}\right)$ é independente de $\mathrm{s}_{0}$, sendo $\mathrm{C}(\mathrm{h})=\mathrm{E}[\mathrm{Z}(\mathrm{s}) \mathrm{Z}(\mathrm{s}+\mathrm{h})]$.

(d) Hipótese de estacionariedade de segunda ordem (Matheron, 1971): Z(s) é dita estacionária de segunda ordem se a variável regionalizada $\mathrm{Z}\left(\mathrm{s}_{0}\right)$ apresenta esperança matemática independente do ponto $\mathrm{s}_{0}$ e se para qualquer vetor h a covariância for $\mathrm{C}(\mathrm{h})=\mathrm{E}[\mathrm{Z}(\mathrm{s}) \mathrm{Z}(\mathrm{s}+\mathrm{h})]-m_{0}^{2}$. Esta hipótese, que não implica na estacionariedade em sensu stricto, é suficiente para a teoria das variáveis regionalizadas. Mas, supõe variância finita a priori.

(e) Variância infinita a priori (Matheron, 1971): muitos fenômenos têm capacidade de dispersão quase ilimitada e não podem ser descritas devidamente atribuindo variância finita a priori. Quando amostras v são retiradas de um campo V, obtémse um histograma que se pode calcular a variância de valores definidos. Esta variância experimental é definida em função de $\sigma^{2}\left(\frac{v}{V}\right)$ do suporte no campo $V$. Se 
as amostras têm variância finita a priori, então isto deve aparecer como uma variância experimental em um campo infinito. Consequentemente, deve-se substituir a estacionariedade de segunda ordem por uma hipótese mais fraca de mesmo significado.

(f) Hipótese Intrínseca (Matheron, 1971): Mesmo quando a variância a priori é infinita, o incremento $\mathrm{Z}\left(\mathrm{s}_{0}+\mathrm{h}\right)-\mathrm{Z}\left(\mathrm{s}_{0}\right)$ pode ter variância finita. Consequentemente, $\mathrm{Z}(\mathrm{s})$ obedece a hipótese intrínseca se, para cada vetor $\mathrm{h}$, o incremento tem uma esperança e variância independentes do ponto s e dependente de h, como:

$$
\mathrm{E}[\mathrm{Z}(\mathrm{s}+\mathrm{h})-\mathrm{Z}(\mathrm{s})]=\mathrm{m}(\mathrm{h})
$$

sendo a função $m(h)$ uma deriva linear. A função

$$
\gamma(h)=1 / 2 E\left[(Z(s+h)-Z(s)]^{2}\right.
$$

é chamada de semivariograma ou função intrínseca. A função aleatória que satisfaz a hipótese intrínseca é caracterizada pelo semivariograma. Se Z(s) obedece a estacionariedade de segunda ordem, ele também obedece a hipótese intrínseca e neste caso:

$$
\gamma(\mathrm{h})=\mathrm{C}(0)-\mathrm{C}(\mathrm{h})
$$

Matheron (1963) explica que os aspectos específicos da distribuição espacial de variáveis regionalizadas devem ser compulsoriamente considerados pela geoestatística e isto é possível pela ferramenta matemática do variograma. Olea (1994) comenta que o semivariograma é uma medida da taxa de mudança pela distância para atributos que variam no espaço. A Figura 1 mostra um variograma estacionário típico e segundo Armstrong (1998) ele apresenta as propriedades: 


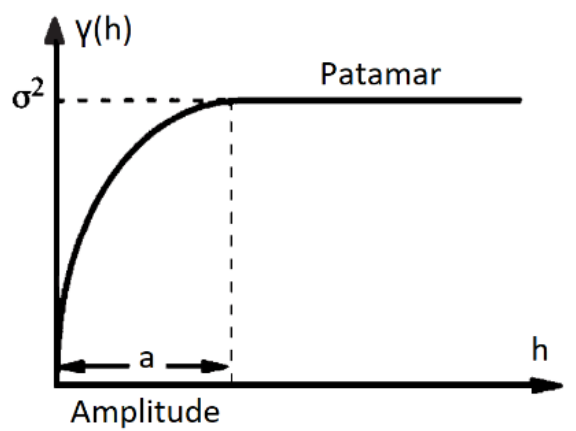

Figura 1- Variograma típico (traduzida de Armstrong, 1998)

(a) Zona de influência (Armstrong, 1998): a taxa de crescimento de um variograma com a distância indica quão rápido a influência de uma amostra sobre outra diminui com a distância. Depois de atingir a variância limite (patamar), não há correlação entre as amostras. Esta distância crítica, chamada de amplitude, dá a definiçãao precisa da noção de zona de influência. A amplitude não precisa ser a mesma para todas as direções, isto indica anisotropia no fenômeno e mesmo para uma determinada direção, pode haver mais de uma amplitude, isto ocorre quando existem diferentes fenômenos com diferentes escalas de distância.

(b) Comportamento próximo a origem (Armstrong, 1998): o estudo do comportamento para valores pequenos de h está relacionado com a continuidade e regularidade espacial da variável. Quatro tipos de comportamento próximo a origem são reconhecidos (Figura 2): (i) quadrático: indica que a variável regionalizada é altamente contínua e é diferenciável. O formato quadrático pode estar associado a presença de deriva; (ii) linear: a variável regionalizada é contínua, mas não diferenciável; (iii) descontínuo próxima a origem (efeito pepita): a variável é muito irregular a curtas distâncias; (iv) pepita puro: puramente aleatória ou ruído branco, as variáveis $\mathrm{Z}(\mathrm{s}+\mathrm{h})$ e $\mathrm{Z}(\mathrm{s})$ não são correlacionadas para valores de $\mathrm{h}$.

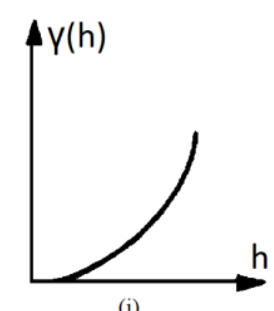

(i)

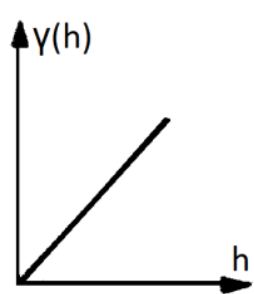

(ii)

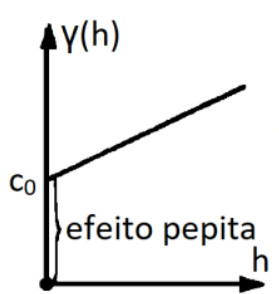

(iii)

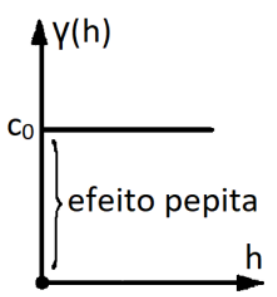

(iv)

Figura 2- Comportamento próximo a origem: (i) quadrático; (ii) linear; (iii) descontinuidade; (iv) pepita puro (traduzida e modificada de Armstrong, 1998) 
(c) Anisotropia (Armstrong, 1998): o variograma calculado em diversas direções pode se comportar de maneira diferente entre elas. Se isto não ocorrer, o variograma depende apenas da distância entre os dois pontos e é chamado de isotrópico. Havendo diferenças de comportamento, o variograma é denominado anisotrópico e a anisotropia pode ser geométrica (a) quando o patamar é o mesmo para todas as direções, porém as amplitudes são distintas; zonal (b) quando os patamares são diferentes e a amplitude é constante e; mista (c) quando a anisotropia é geométrica e zonal, ao mesmo tempo. Estas anisotropias podem ser vistas na Figura 3.

(a) Geométrica

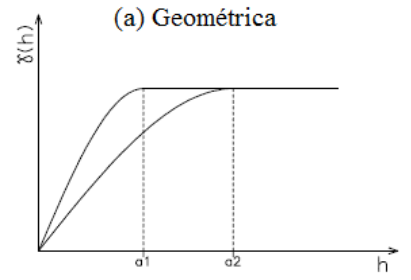

(b) Zonal

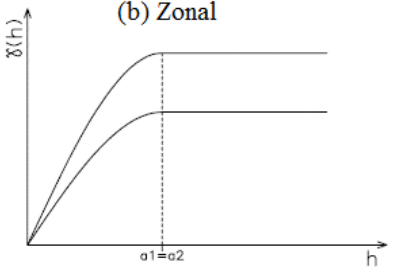

(c) Mista

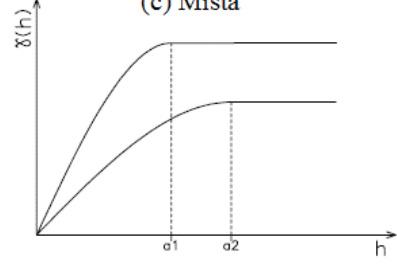

Figura 3 - Anisotropia Geométrica (a), Zonal (b), Mista (c) (Yamamoto, 2001a).

(d) Estruturas imbricadas (Armstrong, 1998): caracterizado pela mudança de curvatura do variograma na zona de influência, isto ocorre quando há fenômenos de diferentes escalas, em uma mesma direção (Figura 4).

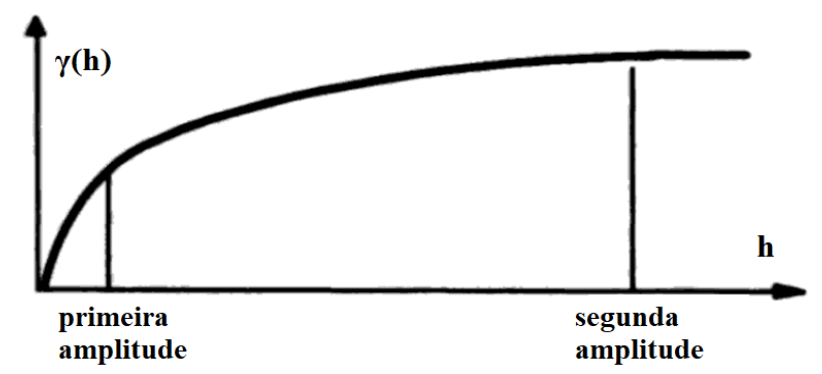

Figura 4 - Estrutura imbricada com duas amplitudes (traduzida e modificada de Armstrong, 1998)

(e) Periodicidade ou efeito furo (Armstrong, 1998): Variogramas podem apresentar crescimento além do patamar e depois queda, formando oscilações periódicas. Pode ser causado por periodicidade de estruturas, dobras ou em variogramas de séries temporais que contém ciclicidade temporal (Figura 5). 


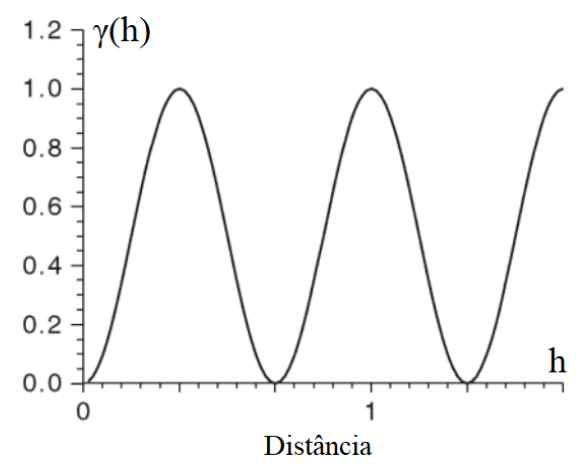

Figura 5 - Variograma com efeito furo (traduzida e modificada de Deutsch, 2002)

Carr (1995) explica que uma das propostas do cálculo do semivariograma é identificar a anisotropia espacial. Para isto, cálculos direcionais são feitos normalmente a cada 4 ou 8 direções (análise exploratória), então se as amplitudes e/ou patamares forem diferentes para cada direção, há anisotropia e se pode definir a direção de maior e menor continuidade espacial. Estas direções são modeladas na forma de uma elipse anisotrópica que é definida pelos eixos perpendiculares de maior e menor continuidade espacial. Caso contrário, trata-se de um fenômeno isotrópico e o cálculo pode ser omnidirecional, em que a direção não é relevante, sendo que os cálculos consideram apenas o incremento (passo). Kitanidis (1997) discorre que a análise dos dados busca duas características estruturais: (i) presença da variabilidade na escala do intervalo de amostragem - depende do comportamento do variograma próximo à origem; (ii) presença de variabilidade na escala do domínio da amostragem - depende do comportamento a longas distâncias.

Na prática, para calcular o semivariograma em uma malha amostral é necessário definir alguns parâmetros:

(a) Azimute: direção do cálculo do variograma;

(b) Número de direções: quantidade de direções em que o semivariograma experimental será calculado;

(c) Tamanho do passo: distância de separação entre amostras que será considerada para o cálculo de cada ponto do semivariograma;

(d) Número de passos: número de vezes que o passo será incrementado. O número de passos vezes o tamanho do passo é a distância máxima considerada no estudo do variograma; 
(e) Tolerância angular: ângulo de busca que define a área de determinado passo para o cálculo do semivariograma;

(f) Tolerância do passo: porcentagem da distância do passo. Esta tolerância faz com que os pares de pontos sejam considerados em classes de distâncias;

(g) Largura máxima: distância perpendicular ao azimute e que define onde a área de busca (de pares do variograma) para de crescer em formato de cone e passa a ser em formato de cilindro, ou seja, a busca tem largura máxima definida.

De acordo com Davis (1986), os semivariogramas experimentais poderiam ser utilizados diretamente para fornecer valores da variância espacial aos processos de estimativa. Entretanto, eles são conhecidos apenas em distâncias múltiplas de h e os semivariogramas devem fornecer variâncias espaciais para qualquer distância. Por isto, o semivariograma experimental deve ser modelado por uma função contínua. O ajuste desta equação é um processo de tentativa e erro, feito interativamente. Então, Armstrong (1998) explica que existem características estatísticas essenciais para que um modelo do variograma possa ser utilizado, por exemplo, deve assegurar que a variância de qualquer combinação linear nunca seja negativa. Uma vez que não é simples reconhecer funções que tenham as propriedades necessárias, o ideal é escolher um modelo de variograma já validado. Estes podem ser somados (como somar funções aleatórias independentes), mas nunca subtraídos. Nem podem ser combinados por partes, ou seja, não pode escolher um modelo até certa distância e outro nas distâncias maiores.

Brooker (1991) comenta que a acurácia do processo de modelagem depende do número de pares de pontos utilizados no cálculo do semivariograma experimental e da distância do passo avaliada. De modo geral, quanto menos pares e quanto maiores forem as distâncias entre amostras menos confiável é o semivariograma. Os modelos de semivariogramas mais comuns podem ser observados em Yamamoto (2001a) e são o esférico, o exponencial e o gaussiano, que podem ser, respectivamente, apresentados na Figura 6(a), (b) e (c) e cujas funções são:

$$
\left\{\begin{array}{c}
\gamma(h)=C_{0}+C\left[\frac{3}{2}\left(\frac{h}{a}\right)-\frac{1}{2}\left(\frac{h}{a}\right)^{3}\right] ; \text { para } \mathrm{h}<a \\
\gamma(h)=C_{0}+C ; \text { para } \mathrm{h} \geq \mathrm{a}
\end{array}\right.
$$




$$
\begin{gathered}
\gamma(h)=C_{0}+C\left[1-\exp \left(-\left(\frac{h}{a}\right)\right)\right] \\
\gamma(h)=C_{0}+C\left[1-\exp \left(-\left(\frac{h}{a}\right)^{2}\right)\right]
\end{gathered}
$$

Sendo: $\gamma(h)$ a variância espacial a um passo de distância $h, C_{0}$ o efeito pepita, $C$ a covariância espacial e $a$ a amplitude do variograma.
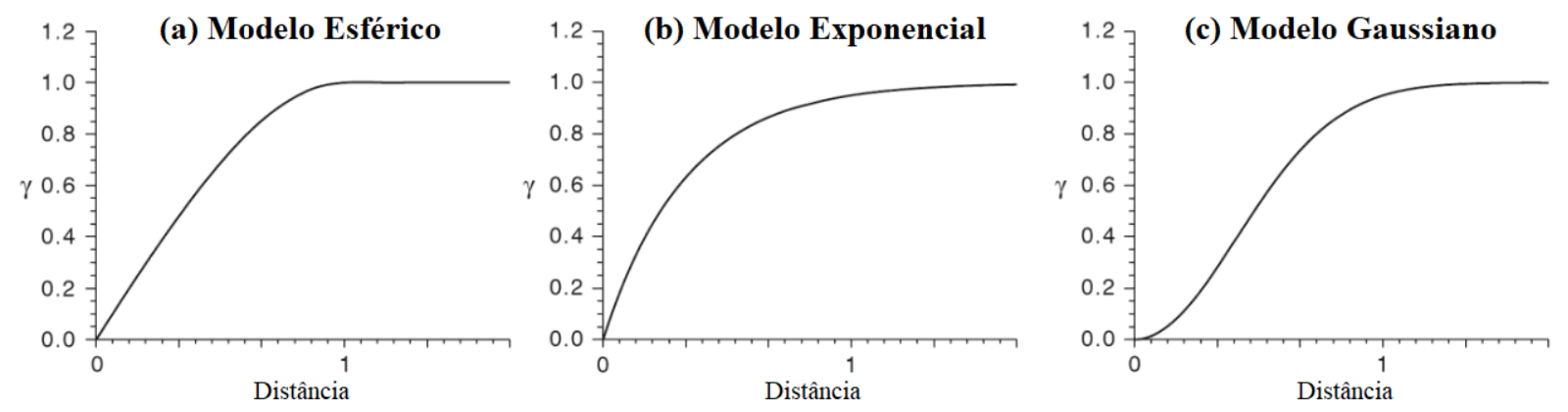

Figura 6 - Os principais modelos teóricos de semivariogramas (traduzida e modificada de Deutsch, 2002)

De acordo com Carr (1995), existem ocasiões que semivariogramas não podem ser modelados facilmente e diferentes modelos podem existir para diferentes escalas, a correlação espacial múltipla pode ser representada utilizando combinações lineares dos modelos de semivariogramas. Olea (1994) explica que a adição de modelos é uma propriedade admissível dos modelos de semivariogramas. Qualquer combinação linear de modelos admissíveis com coeficientes positivos é permitida e isto é chamado de semivariograma imbricado.

Para a etapa de estimativa ou simulação, devem-se escolher parâmetros de vizinhança, estes parâmetros dependem do algoritmo implementado no programa. Se algum destes parâmetros não for satisfeito, o ponto não será estimado ou simulado. Os principais parâmetros são:

(a) Número mínimo e máximo de amostras utilizadas;

(b) Divisão do espaço amostral em setores (quadrantes ou octantes) para garantir uma boa disposição espacial das amostras utilizadas e evitar concentrações preferenciais. 
A Figura 7 mostra o cenário com critério apenas baseado na distância, a divisão de quadrantes e divisão de octantes;
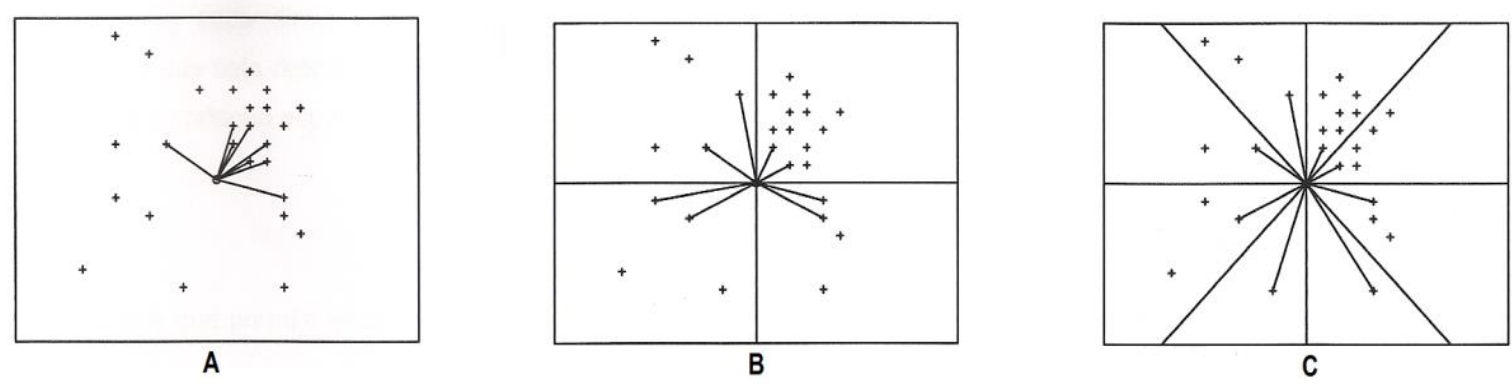

Figura 7 - Comparação entre os métodos de seleção de amostras. A figura A mostra a captura de dados com os pontos mais próximos; A figura B mostra a captura de dois dados para cada quadrante e a $\mathrm{C}$ mostra um dado para cada octante (modificada de Yamamoto, 2001b)

(c) Número máximo e mínimo de amostras por setores;

(d) Número aceitável de setores sem amostras;

(e) Elipse de busca para limitar a distância máxima entre o ponto sendo estimado e as amostras utilizadas na estimativa. Normalmente esta elipse é diretamente relacionada à elipse que representa a anisotropia do variograma.

Uma maneira de verificar a qualidade do ajuste do modelo teórico de variograma e a vizinhança de busca definida é aplicar a validação cruzada dos dados. Este procedimento remove um valor amostrado do banco de dados, o estima e compara o valor estimado com o real. Quando é feito para todos os pontos do banco de dados é possível fazer um gráfico de dispersão entre os valores reais e os estimados.

\subsubsection{Krigagem simples}

Segundo Deutsch e Journel (1998), a krigagem é uma coleção de técnicas de regressão linear para minimizar a variância do erro da estimativa. Considerando a estimativa de um valor $\mathrm{z}\left(\mathrm{s}_{0}\right)$ de uma vizinhança $\mathrm{z}\left(\mathrm{s}_{\mathrm{i}}\right), \mathrm{i}=1, \ldots, \mathrm{n}$, a função aleatória $\mathrm{Z}(\mathrm{s})$ é estacionária com média $\mathrm{m}$ e covariância C(h) (ou semivariância espacial $\gamma(h)$ ). Na maneira mais trivial, a krigagem simples (SK) considera o estimador 


$$
\mathrm{Z}_{\mathrm{SK}}^{*}\left(\mathrm{~s}_{0}\right)=\sum_{\mathrm{i}=1}^{\mathrm{n}} \lambda_{\mathrm{i}} \mathrm{Z}\left(\mathrm{x}_{\mathrm{i}}\right)+\left(1-\sum_{\mathrm{i}=1}^{\mathrm{n}} \lambda_{\mathrm{i}}\right) \mathrm{m}
$$

O peso $\lambda$ é determinado para a variância mínima do erro, chamada de variância da estimativa que minimiza o resultado em um conjunto de equações

$$
\sum_{j=1}^{\mathrm{n}} \lambda_{\mathrm{i}} \mathrm{C}_{\mathrm{ij}}=\mathrm{C}_{\mathrm{i} 0}, \quad \forall \mathrm{i}=1, \ldots, \mathrm{n}
$$

Goovaerts (1997) mostra que, em notação matricial, o sistema de krigagem simples é descrito como:

$$
\left[\begin{array}{cccc}
\mathrm{C}_{11} & \mathrm{C}_{12} & \ldots & \mathrm{C}_{1 n} \\
\mathrm{C}_{21} & \mathrm{C}_{22} & \ldots & \mathrm{C}_{2 n} \\
\vdots & \vdots & \ldots & \vdots \\
\mathrm{C}_{n 1} & \mathrm{C}_{n 2} & \ldots & \mathrm{C}_{n n}
\end{array}\right] \cdot\left[\begin{array}{c}
\lambda_{1} \\
\lambda_{2} \\
\vdots \\
\lambda_{n}
\end{array}\right]=\left[\begin{array}{c}
\mathrm{C}_{10} \\
\mathrm{C}_{20} \\
\vdots \\
\mathrm{C}_{n 0}
\end{array}\right]
$$

Onde $\mathrm{C}_{\mathrm{ij}}$ é a covariância espacial entre as amostras da vizinhança, $\mathrm{C}_{\mathrm{i} 0}$ é a covariância espacial entre as amostras e o ponto a estimar e $\lambda_{i}$ são os pesos da krigagem simples. Deutsch e Journel (1998) escrevem a variância de krigagem (variância da estimativa minimizada) como

$$
\sigma_{S K}^{2}=\mathrm{C}(0)-\sum_{i=1}^{n} \lambda_{i} C_{i 0} \geq 0
$$

Onde $\mathrm{C}(0)$ é a covariância espacial na distância 0 . 


\subsubsection{Krigagem ordinária}

De acordo com Isaaks e Srivastava (1989), a krigagem ordinária é conhecida como B.L.U.E. (best linear unbiased estimator) que significa o melhor estimador linear não enviesado. Os autores explicam que é linear pois estima com base em pesos de combinações lineares dos dados, que é não enviesado porque tem resíduo médio igual a zero e melhor por minimizar a variância dos erros $\left(\sigma_{R}^{2}\right)$. Na krigagem ordinária, utiliza-se um modelo de probabilidade para estimar valores desconhecidos, em cada ponto, onde não há amostra. $\mathrm{O}$ viés e a variância do erro podem ser calculados e os pesos para as amostras escolhidos, por combinações lineares, de modo que garantam que a média do erro seja zero e sua variância minimizada. Cada variável aleatória tem a mesma lei de probabilidade em todos os locais, o valor esperado da variável aleatória é $\mathrm{E}\{\mathrm{Z}(\mathrm{s})\}$ e qualquer par de variáveis aleatórias tem distribuição conjunta que depende apenas da separação entre os pontos e não de suas posições. Portanto, a covariância entre pares de variáveis aleatórias, separadas pela distância h é C(h). Os mesmos autores ainda mostram que cada valor amostrado é uma variável aleatória, então a estimativa também é uma variável aleatória, desde que seja a combinação linear dos pesos $\lambda$ das amostras, como:

$$
\mathrm{Z}\left(\mathrm{s}_{0}\right)=\sum_{i=1}^{n} \lambda_{i} Z\left(s_{i}\right)
$$

Sendo n o número de amostras vizinhas utilizadas $\left(\mathrm{Z}\left(\mathrm{s}_{\mathrm{i}}\right)\right)$ e $\lambda$ os pesos da combinação linear. O erro estimado definido como estimativa e variável aleatória modelada é

$$
\mathrm{R}\left(\mathrm{s}_{0}\right)=\sum_{i=1}^{n} \lambda_{i} Z\left(s_{i}\right)-Z\left(s_{0}\right)
$$

Considerando que o erro em qualquer local particular tem esperança 0 e que a variável aleatória é estacionária de segunda ordem: 


$$
\begin{gathered}
\mathrm{E}\left[\mathrm{R}\left(\mathrm{s}_{0}\right)\right]=\mathrm{E}\left[\sum_{i=1}^{n} \lambda_{i} Z\left(s_{i}\right)-Z\left(s_{0}\right)\right]=0 \\
\Rightarrow \sum_{i=1}^{n} \lambda_{i}=1
\end{gathered}
$$

Isaaks e Srivastava (1989) ainda explicam que se introduz o parâmetro de Lagrange $\mu$, o que não afeta a equidade e converte o problema de minimização de restrições em um problema irrestrito. Então o modelo de $\sigma_{R}^{2}$ é função de $n+1$ variáveis, n pesos e 1 parâmetro de Lagrange. Ao minimizar a variância dos erros o sistema de equações da krigagem ordinária é, em sua forma matricial:

$$
\left[\begin{array}{ccccc}
\gamma_{11} & \gamma_{12} & \ldots & \gamma_{1 n} & 1 \\
\gamma_{21} & \gamma_{22} & \ldots & \gamma_{2 n} & 1 \\
\vdots & \vdots & \ldots & \vdots & \vdots \\
\gamma_{n 1} & \gamma_{n 2} & \ldots & \gamma_{n n} & 1 \\
1 & 1 & \ldots & 1 & 0
\end{array}\right] \cdot\left[\begin{array}{c}
\lambda_{1} \\
\lambda_{2} \\
\vdots \\
\lambda_{n} \\
\mu
\end{array}\right]=\left[\begin{array}{c}
\gamma_{10} \\
\gamma_{20} \\
\vdots \\
\gamma_{n 0} \\
1
\end{array}\right]
$$

Para minimizar o modelo de variâncias do erro, deve-se escolher $(n+1)^{2}$ covariâncias (ou variâncias) que descrevam a continuidade espacial do modelo de função aleatória, construindo as matrizes onde $\gamma_{\mathrm{ij}}$ são variâncias espaciais entre amostras e $\gamma_{\mathrm{n} 0}$ são as variâncias espaciais entre as amostras e o ponto desconhecido e $\mu$ é o multiplicador de Lagrange (Isaaks e Srivastava, 1989). Os mesmos autores ainda definem a equação da variância de krigagem ordinária como:

$$
\sigma_{K O}^{2}=\sum_{i=1}^{n} \lambda_{i} \gamma_{i 0}+\mu
$$

\subsubsection{Simulação geoestatística}

Hohn (1997) explica que uma vantagem importante para o mapeamento pela abordagem geoestatística é a modelagem da covariância espacial. Os modelos teóricos de semivariogramas fazem a estimativa ser sensível a anisotropia dos dados. Por outro lado, a suavização da krigagem elimina os detalhes dos mapas. Olea (1999) ressalta que a geoestatística tenta 
solucionar a suavização da krigagem por meio de simulação estocástica. Infelizmente, a simulação é apenas uma troca, uma vez que se ganha em precisão global, porém se perde em precisão local. Realizações estocásticas não estão livres de erros e interpretações da realidade. $\mathrm{Na}$ falta de uma solução perfeita, a escolha entre krigagem e simulação deve ser decidida com base no que é mais relevante para cada aplicação específica: o erro de estimativa local minimizado (precisão local) obtido pela krigagem ou a distribuição dos dados e a continuidade espacial correta (precisão global) obtida pela simulação estocástica, que, por sua vez, assume que cada realização é equiprovável e o histograma da amostra é bom o suficiente para representar o histograma real, ou seja, que a amostragem fornece boa aproximação do real.

De acordo com Pyrcz et al. (2006), as simulações geoestatísticas estão sendo utilizadas cada vez mais para quantificar as incertezas. As técnicas de simulação reproduzem as estatísticas de dados com flutuações ergódigas. A extensão que as estatísticas são reproduzidas dependem quase exclusivamente do tamanho do domínio relativo à amplitude da correlação espacial. Myers (2006) discorre que, pela sua natureza, os métodos de simulação não podem produzir uma resposta absolutamente correta, mas o processo gera uma boa aproximação com uma medida de incerteza associada ao resultado. Em resumo, a simulação estocástica é um processo que constrói alternativas equiprováveis e modelos de distribuição espacial com alta resolução. Ela é dita condicional se o resultado das realizações honrarem os valores dos dados e sua localização (Deutsch e Journel, 1998).

Goovaerts (2006) mostra que o conjunto de simulações pode ser utilizado para derivar medidas de incertezas de complexidades crescentes conforme mais pontos são envolvidos no cálculo. Segundo Lantuejoul (2002), uma sequência de valores é considerada como simulada se ela compartilha as mesmas propriedades estatísticas como uma sequência de realizações de variáveis mutuamente independentes da distribuição. Gotway e Rutherford (1994) explicam que as simulações geoestatísticas geram realizações com estatísticas e propriedades geoestatísticas específicas. Elas são sugeridas como métodos viáveis para caracterizar as incertezas associadas a estimativas de parâmetros espaciais de funções não lineares. Uma função não linear (chamada de função de transferência) é avaliada sobre cada realização para se obter a distribuição de incertezas que reflete a variabilidade espacial e a incerteza do parâmetro.

Srivastava (1994) mostra que a família de procedimentos sequenciais segue o mesmo algoritmo básico e que a diferença significativa, na prática, é como a distribuição probabilística 
condicional local é estimada. Chilès e Delfiner (1999) demonstram que se pode simular o vetor $\mathrm{Z}$ sequencialmente por uma seleção de $\mathrm{Z}_{\mathrm{i}}$ a partir de uma distribuição condicional $\mathrm{P}\left\{\mathrm{Z}_{\mathrm{i}}<\mathrm{Z}_{\mathrm{i}}\right.$, ...,Zi-1 $\}$ para $\mathrm{i}=\mathrm{M}+1, \ldots, \mathrm{N}$ e incluir o resultado simulado $\mathrm{Zi}$ em um banco de dados condicional para a simulação dos próximos pontos dentro da mesma realização. Os autores ainda ressaltam que o processo pode ser utilizado em particular com $Z_{i}$ de forma que $Z_{i}=Z\left(s_{i}\right)$, onde $Z(s)$ é uma função aleatória, Si são os pontos amostrados $(i=1,2, \ldots, M)$ e os pontos onde se deseja simular a função aleatória $(i=M+1, \ldots, N)$. Isto possibilita a construção de uma simulação não condicional $(\mathrm{M}=0)$ e uma simulação condicional $(\mathrm{M}>0)$.

Segundo Chilès e Delfiner (1999), uma simulação não condicional da função aleatória $\left\{\mathrm{Z}(\mathrm{s}): \mathrm{s} \in \mathrm{R}^{\mathrm{n}}\right\}$ é simplesmente uma realização de $\mathrm{Z}(\mathrm{s})$ selecionada aleatoriamente de um conjunto de todas as possíveis realizações e ela depende apenas das distribuições espaciais de $\mathrm{Z}(\mathrm{s})$. Por outro lado, uma simulação condicional tem número finito de realizações e assume os mesmos valores que os observados nos pontos amostrados, assim estes podem ser considerados para representar melhor a variável regionalizada $\mathrm{z}(\mathrm{s})$. Os mesmos autores ainda comentam que estas simulações condicionais são qualitativamente úteis para manter a distribuição real da variabilidade espacial. Enquanto, quantitativamente ela pode avaliar a incerteza espacial dos resultados de procedimentos complexos, i.e., modelagem de um sistema dinâmico ou a otimização econômica do desenvolvimento de uma fonte natural. A simulação condicional tem como objetivo a manutenção das variações espaciais de maneira mais realista possível, ela não reproduz os mecanismos genéticos que criam o fenômeno em estudo, sendo assim, seus resultados devem ser analisados com um olhar crítico específico para cada aplicação (Chilès e Delfiner, 1999).

Chilès e Delfiner (1999) explicam detalhadamente que existem algoritmos de simulação geoestatística (e.g. bandas rotativas) que não são condicionais a priori e para realizar uma simulação estocástica condicional, deve-se considerar uma função aleatória Z(s) conhecida com $N$ amostras $S_{i}, i=1,2, \ldots, N$. Sendo assim, os autores mostram que uma simulação não condicional S(s) é independente de Z(s), porém ambas têm a mesma covariância espacial. E para condicionar esta simulação é necessário fixar os pontos amostrados através de uma operação que transforma a simulação não condicional S(s) para uma simulação condicional $\mathrm{S}_{\mathrm{c}}(\mathrm{s})$. Desta maneira, considere $\mathrm{Z}^{*}(\mathrm{~s})$ como o estimador da krigagem de $\mathrm{Z}(\mathrm{s})$ no ponto $\mathrm{s}$, baseado nos dados $\mathrm{Z}\left(\mathrm{S}_{\mathrm{i}}\right)$ : 


$$
\mathrm{Z}(\mathrm{s})=\mathrm{Z}^{*}(\mathrm{~s})+\left[\mathrm{Z}(\mathrm{s})-\mathrm{Z}^{*}(\mathrm{~s})\right]
$$

Sendo Z(s) desconhecido, o erro da krigagem não tem como ser calculado, portanto considerando a igualdade para $S(s)$, onde $S^{*}(s)$ é o estimador da krigagem obtido como se a simulação fosse conhecida apenas no ponto $\mathrm{s}_{0}$ :

$$
S(s)=S^{*}(s)+\left[S(s)-S^{*}(s)\right]
$$

O valor verdadeiro de $S(s)$ é conhecido e, consequentemente, seu erro será $S(s)-S^{*}(s)$. Com a decomposição de Z(s), o erro desconhecido - pela simulação deste erro - gera a simulação condicional $\mathrm{S}_{\mathrm{c}}(\mathrm{s})$ definida por Chilès e Delfiner (1999) como o estimador da krigagem $\left(\mathrm{Z}^{*}(\mathrm{~s})\right)$ mais a simulação do erro da krigagem $\left(\left[S(s)-S^{*}(s)\right]\right)$, ou seja:

$$
\mathrm{S}_{\mathrm{c}}(\mathrm{s})=\mathrm{Z}^{*}(\mathrm{~s})+\left[\mathrm{S}(\mathrm{s})-\mathrm{S}^{*}(\mathrm{~s})\right]
$$

Chilès e Delfiner (1999) mostram que a média de uma grande quantidade de realizações condicionais independentes em um determinado ponto tende ao valor estimado pela krigagem. É esperado que a simulação condicional se comporte como o campo real, mas sem estimá-lo. Journel e Huijbregts (1978) mostram que em termos de variância da estimativa, a krigagem é duas vezes melhor que a simulação condicional, portanto, a estimativa não é o objetivo da simulação. O princípio de condicionamento leva em consideração a independência dos erros e a variância da estimativa de valores reais $\mathrm{Z}_{0}(\mathrm{~s})$ na simulação condicional pode ser escrita como $\mathrm{E}\left[\mathrm{S}_{\mathrm{c}}(\mathrm{s})-\mathrm{Z}(\mathrm{s})\right]^{2}=2 \sigma^{2}$.

Lantuejoul (2002) salienta que uma consequência extremamente útil do teorema do limite central é a existência de uma classe de funções cuja distribuição espacial depende apenas dos 2 primeiros momentos, estas são as funções aleatórias gaussianas. Deutsch (2002) comenta que as simulações nos espaços gaussianos (e.g. Simulação Sequencial Gaussiana) apresentam propriedades matemáticas não são limitadas à sua distribuição gaussiana. Isto é, 
independentemente da distribuição dos dados, a reprodução da covariância da krigagem se mantém e a correção da variância é feita com a adição de um resíduo aleatório que funciona de maneira independente do formato de sua distribuição. O autor ainda explica que o melhor motivo para utilizar a distribuição gaussiana é que qualquer outra distribuição não leva a uma distribuição global correta dos valores simulados. Ou seja, as estatísticas de primeira e segunda ordens, como a média, variância e variograma dos dados, podem estar corretos, mas a forma da distribuição não. Entretanto, no espaço gaussiano, todas as distribuições são gaussianas. Além disso, o teorema do limite central diz que a adição sequencial de resíduos aleatórios para obter valores simulados conduz à distribuição gaussiana.

Chilès e Delfiner (1999) explicam que em regiões não estacionárias - como um reservatório de petróleo, por exemplo - uma única realização fornece apenas uma possibilidade em termos de fluxo e produção e, desta forma, a simulação de diversas realizações é necessária para se conhecer a gama dos possíveis resultados. Os autores ainda comentam que geralmente 100 realizações são consideradas uma boa quantidade para avaliações estatísticas e funções de transferência, pois quando são feitas diversas realizações para cada simulação da função aleatória, cada uma leva a um resultado semelhante, porém com flutuações. Se os dados estiverem enviesados e/ou se houver fundamental interesse nos valores extremos, um maior número de realizações é necessário, ressaltando que as previsões de valores extremos geralmente não possuem robustez estatística na estimativa dos valores. Portanto, o número ideal de realizações depende muito do interesse nos parâmetros de distribuição.

\subsubsection{Simulação por bandas rotativas}

Chilès e Delfiner (1999) explicam que alguns modelos de covariância podem ser simulados diretamente no $\mathrm{R}^{\mathrm{n}}$. Mas é mais simples utilizar o método de bandas rotativas que permite a construção da simulação no espaço a partir de simulações em linhas, ou seja, reduz simulações multidimensionais para simulações unidimensionais (Lantuejoul, 2002). Chilès e Delfiner (1999) ainda comentam que os métodos de simulação em uma linha são de grande interesse para o método de bandas rotativas, mesmo quando o objetivo é simular em 2D ou 3D. O método de bandas rotativas consiste em adicionar um grande número de simulações independentes definidas nas linhas que varrem o plano. A Figura 8 representa o princípio do método em duas dimensões (plano). Os mesmos autores ainda explicam que o valor da 
simulação em um ponto s do plano é a soma de seus valores assumidos na projeção de $\mathrm{s}$ nas diferentes linhas que correspondem as simulações unidimensionais.

Lantuejoul (2002) explica que o método considera um sistema de coordenadas polares $\mathrm{u}=(\theta, \rho)$ onde $\theta$ é o parâmetro de direção e $\rho$ é o parâmetro de localização para a criação das linhas em que ocorrem as simulações. Assim, a estimativa pode ser escrita como função da distribuição de $\theta$ e a distribuição condicional de $\rho$ dado $\theta$. A covariância $C$ pode ser considerada como uma função unidimensional $\left(\mathrm{C}_{\theta}\right)$. A ideia por trás da simulação de bandas rotativas é reduzir a simulação de uma função aleatória gaussiana com covariância $C$ para uma simulação de processos estocásticos independentes com covariância $C_{\theta}$. Sendo $\theta_{n}(n \in N)$ uma sequência de direções de $S_{d}^{+}$e $\left(\mathrm{X}_{\mathrm{n}}, \mathrm{n} \in \mathrm{N}\right)$ uma sequência de processos estocásticos independentes com covariância $\mathrm{C}_{\theta}$. Então a função aleatória

$$
S^{(n)}(s)=\frac{1}{\sqrt{n}} \sum_{k=1}^{n} X_{k}\left(<x, \theta_{k}>\right) \quad x \in R^{d}
$$

Admite a covariância

$$
C^{(n)}(h)=\frac{1}{n} \sum_{k=1}^{n} C_{\theta_{k}}\left(<h, \theta_{k}>\right)
$$

Quando $\mathrm{n}$ se torna um valor muito alto, o teorema do limite central implica que a distribuição de $S^{(n)}$ tende a se tornar gaussiana com covariância $\lim _{n \rightarrow+\infty} C^{(n)}$. 


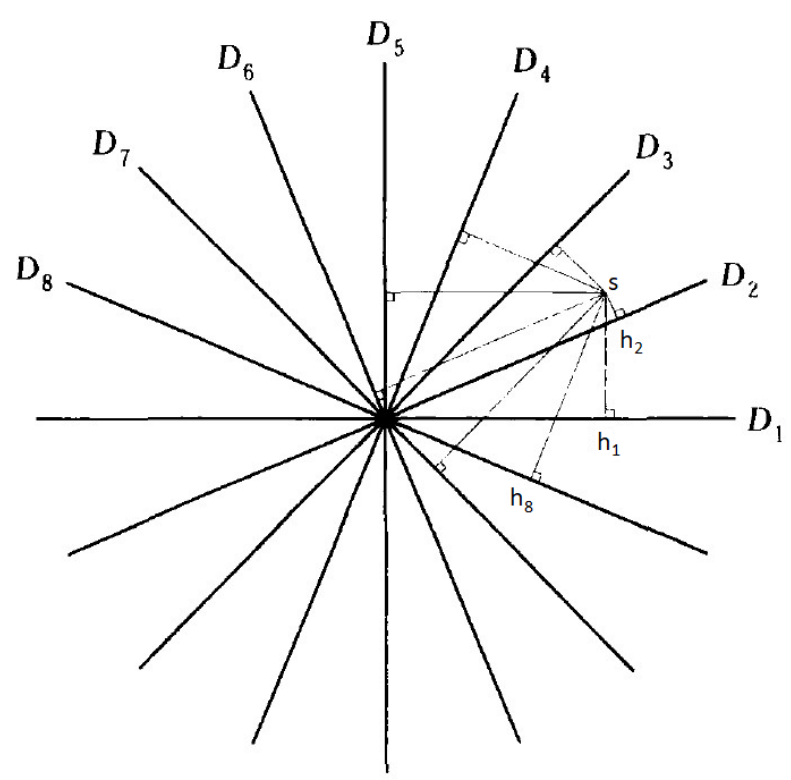

Figura 8 - O princípio do método de bandas rotativas em 2D (Chilès e Delfiner, 1999)

Lantuejoul (2002) definiu o algoritmo do método de bandas rotativas como:

a) Escolher um conjunto de direções $\theta_{1}, \ldots, \theta_{n}$

b) Gerar um processo estocástico padrão independente $X_{1}, \ldots, X_{n}$ com covariância $C_{\theta_{1}}, \ldots, C_{\theta_{n}}$

c) Calcular a função aleatória (Eq. 19)

E etapa b e c vem da aplicação do teorema do limite central.

A simulação feita por bandas rotativas é não condicional e Deutsch e Journel (1998) evidenciam que o condicionamento da simulação para o local real exige um pós-processamento da simulação não condicional através de krigagens. Neste método de simulação é necessário escolher o número de bandas rotativas que serão utilizadas na simulação e quando o número de bandas é pequeno os resultados apresentam artefatos, conforme pode ser observado na Figura 9 onde estão exemplificados os resultados dessa simulação calculada com 1, 2 e 3 bandas. 


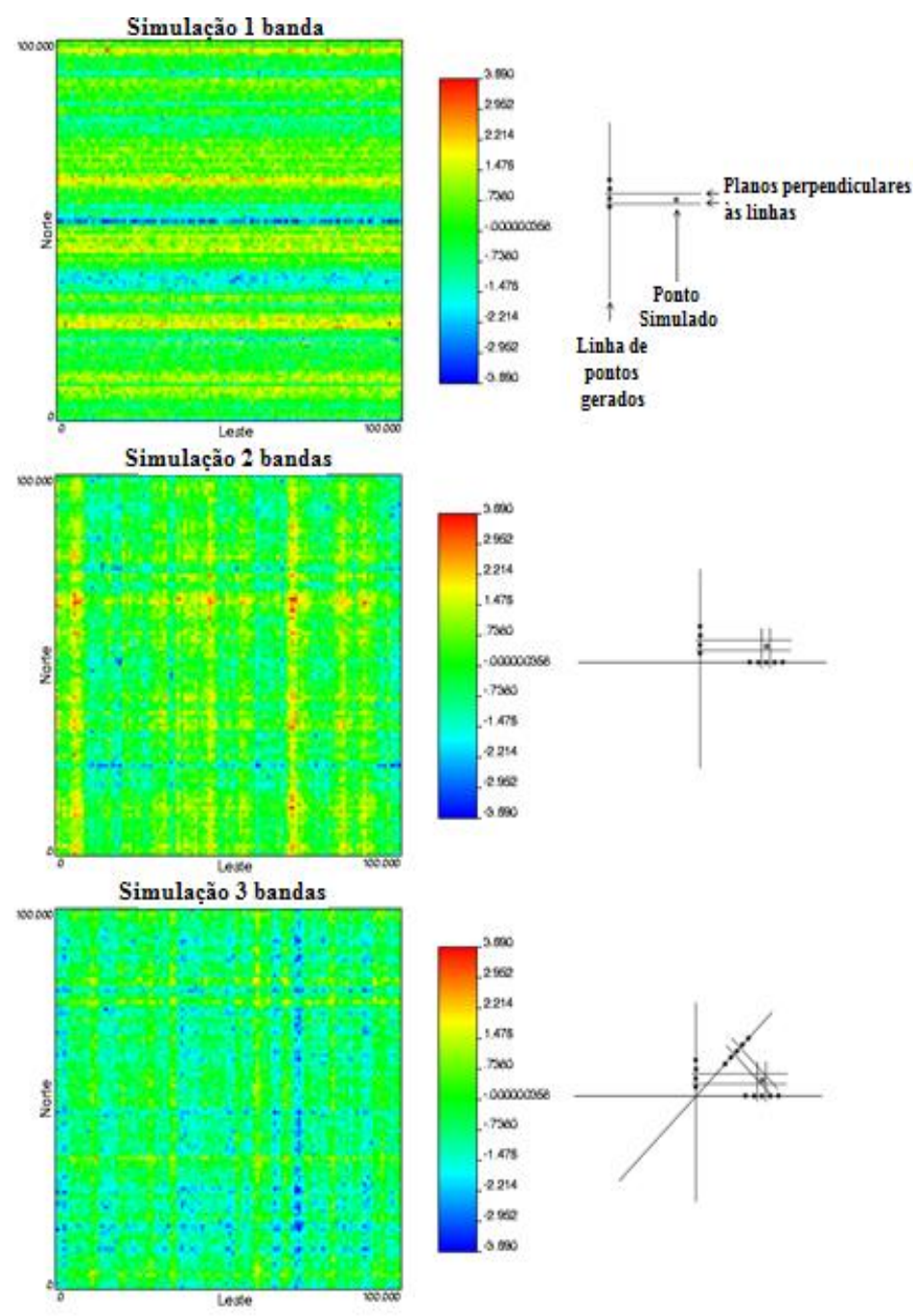

Figura 9 - Imagens resultantes de simulações com 1, 2 e 3 bandas comparadas com esquemas ilustrativos da contribuição de cada banda (Watanabe, 2003)

\subsection{Geoestatística Espaçotemporal}

De acordo com Chilès e Delfiner (1999), o estudo de múltiplas séries temporais localizadas no espaço - ou seja, variáveis $\mathrm{Z}(\mathrm{s}, \mathrm{t}$ ) com localização no espaço s e no tempo $\mathrm{t}$ podem ser tratadas como diversas realizações de um campo aleatório espacial em que o tempo é um índice replicante. A existência destes replicantes permite a inferência de modelos não estacionários, o que leva a melhores estimativas espaciais. Assim, pode-se estudar questões espaçotemporais importantes como atividade humana na saúde ou aquecimento global, com o cálculo em uma região e em diferentes tempos, assim como suas tendências e a quantificação de sua incerteza. Matematicamente, a variável Z(s, t) pode ser considerada como uma função aleatória no espaço dimensional $\mathrm{R}^{\mathrm{n}} \mathrm{x} \mathrm{T}$, com $\mathrm{n}$ dimensões espaciais e uma dimensão temporal. 
Se for possível calcular um modelo da estrutura de covariância e a deriva no espaço, então a krigagem segue como usual.

Snepvangers et al. (2003) mostram que o espaço representa um estado de coexistência, em que pode haver múltiplas dimensões e normalmente a interpolação é o objetivo. Enquanto o tempo, representa o estado de existência sucessiva, ou seja, uma dimensão não reversível onde normalmente o objetivo é a extrapolação. Além disso, a origem das variações no espaço e no tempo podem ter fontes diferentes (por exemplo: na agricultura, o tempo pode ser dominado pela precipitação e o espaço pelas propriedades físicas do solo e vegetação) podendo caracterizar a "anisotropia".

Kyriakidis e Journel (1999) explicam que existe uma ordem nos dados temporais - do passado para o presente - que não acontece em observações espaciais. Anisotropia (ou isotropia) é bem definida no espaço, enquanto não há significado no contexto espaçotemporal devido ao ordenamento intrínseco e irreversível do tempo. Os autores ainda mostram que as escalas e unidades são diferentes no espaço e no tempo e fisicamente não podem ser comparadas. Portanto, a combinação dos domínios do espaço e do tempo devem ser feitas apenas como um sistema de coordenadas, onde as observações são representadas por um vetor com coordenadas de localização s e coordenada temporal t. A Figura 10 ilustra duas maneiras de estudar as variáveis espaçotemporais, onde a Figura 10A representa a variação encontrada em uma região em 3 tempos diferentes, ou seja, existe uma função aleatória para cada tempo. A segunda maneira (Figura 10B) simboliza pontos fixos no espaço que tem uma componente temporal variável como vetores de séries temporais, ou seja, cada ponto amostrado tem valores diferentes para cada momento no tempo e períodos não registrados podem ter seu perfil reconstruído por modelos de séries temporais. 


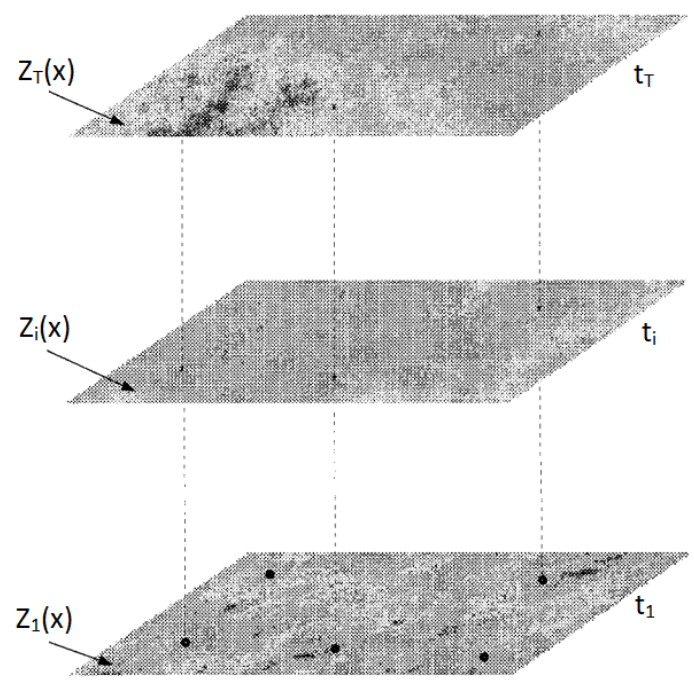

(A)

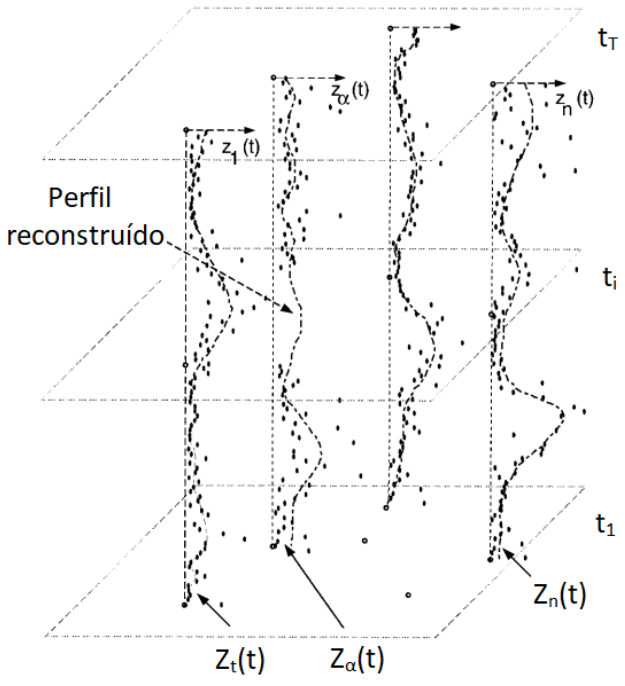

(B)

Figura 10- Em (A) o modelo conceitual para vetores de uma função aleatória Zi(s), i = 1, ...,T e em (B) a representação de vetores de uma série temporal $Z \alpha(\mathrm{t}), \alpha=1, \ldots, \mathrm{n}$ (traduzida e modificada de Kyriakidis e Journel, 1999)

Geralmente, as amostras espaçotemporais têm uma localização fixa no espaço e frequência de coleta constante. Como, por exemplo, dados de estações meteorológicas que são armazenados como médias diárias. Neste caso, uma maneira simples de ilustrar a estrutura espaçotemporal dos dados é um cubo espaçotemporal como pode ser visto na Figura 11.

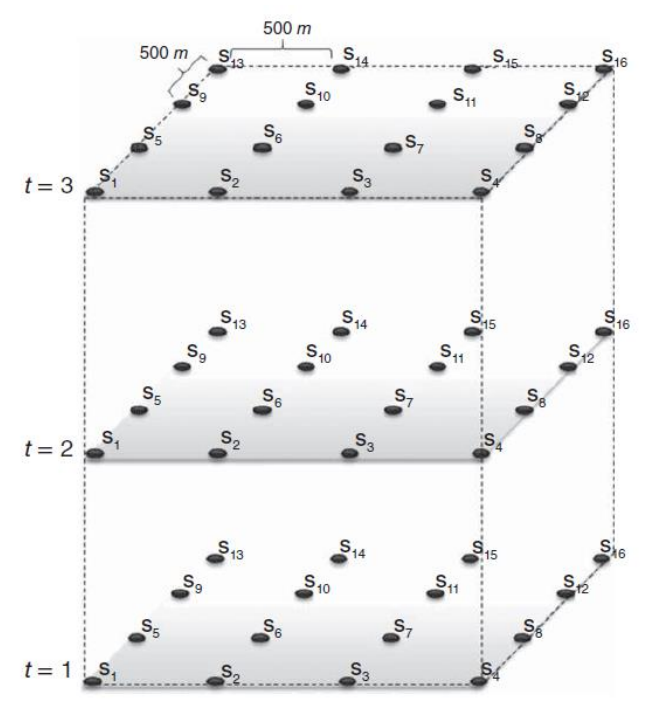

Figura 11 - Cubo espaçotemporal. Amostras $S_{\mathrm{i}}$ são regulares em 500 x 500 metros e foram coletadas em três momentos no tempo (Montero et al., 2015) 
A covariância entre $\mathrm{Z}\left(\mathrm{s}_{1}, \mathrm{t}_{1}\right)$ e $\mathrm{Z}\left(\mathrm{s}_{2}, \mathrm{t}_{2}\right)$ depende das suas coordenadas. Entretanto, na prática, tanto a estimativa quanto a modelagem assumem simplificações, como estacionariedade, separabilidade e simetria (Gneiting et al., 2007). Montero et al. (2015) caracterizam algumas das principais classes de funções das variáveis espaçotemporais, como:

(a) Função covariância espacial estacionária (Montero et al., 2015): para quaisquer dois pares de pontos $\left(\mathrm{s}_{\mathrm{i}}, \mathrm{t}_{\mathrm{i}}\right)$ e $\left(\mathrm{s}_{\mathrm{j}}, \mathrm{t}_{\mathrm{j}}\right)$ a covariância $\mathrm{C}\left(\left(\mathrm{s}_{\mathrm{i}}, \mathrm{t}_{\mathrm{i}}\right),\left(\mathrm{s}_{\mathrm{j}}, \mathrm{t}_{\mathrm{j}}\right)\right)$ depende apenas da distância entre as localidades $\left(s_{i}-s_{j}\right)$ e do tempo $t_{i}$ e $t_{j}$.

(b) Função covariância temporal estacionária (Montero et al., 2015): para quaisquer dois pares de pontos $\left(s_{i}, t_{i}\right)$ e $\left(s_{j}, t_{j}\right)$, a covariância $C\left(\left(s_{i}, t_{i}\right),\left(s_{j}, t_{j}\right)\right)$ depende apenas da distância entre os tempos $\left(t_{i}-t_{j}\right)$ e da localização espacial $s_{i}$ e $s_{j}$.

(c) Função covariância estacionária (Montero et al., 2015): a função covariância estacionária espacial e temporal é expressa como:

$$
\mathrm{C}\left(\left(\mathrm{s}_{\mathrm{i}}, \mathrm{t}_{\mathrm{i}}\right),\left(\mathrm{s}_{\mathrm{j}}, \mathrm{t}_{\mathrm{j}}\right)\right)=\mathrm{C}(\mathrm{h}, \mathrm{u})
$$

$\operatorname{com~} h=s_{i}-s_{j}$ e $u=t_{i}-t_{j}$ sendo as distâncias no espaço e no tempo, respectivamente.

(d) Função covariância separável (Montero et al., 2015): existe se há uma função covariância puramente espacial $\mathrm{C}_{\mathrm{s}}\left(\mathrm{s}_{\mathrm{i}}, \mathrm{s}_{\mathrm{j}}\right)$ e uma função covariância temporal $\mathrm{C}_{\mathrm{t}}\left(\mathrm{t}_{\mathrm{i}}, \mathrm{t}_{\mathrm{j}}\right)$ que garanta:

$$
C\left(\left(s_{i}, t_{i}\right),\left(s_{j}, t_{j}\right)\right)=C_{s}\left(s_{i}, t_{i}\right) C_{t}\left(s_{j}, t_{j}\right)
$$

para cada par de localizações espaçotemporais $\left(\mathrm{s}_{\mathrm{i}}, \mathrm{t}_{\mathrm{i}}\right)$ e $\left(\mathrm{s}_{\mathrm{j}}, \mathrm{t}_{\mathrm{j}}\right) \in \mathrm{R}^{\mathrm{n}} \mathrm{x}$ T. Se isto não ocorrer, a função covariância é chamada de não-separável.

(e) Função covariância inteiramente simétrica (Montero et al., 2015):

$$
C\left(\left(s_{i}, t_{i}\right),\left(s_{j}, t_{j}\right)\right)=C\left(\left(s_{i}, t_{j}\right),\left(s_{j}, t_{i}\right)\right)
$$

para cada par de localizações espaçotemporais $\left(s_{i}, t_{i}\right)$ e $\left(s_{j}, t_{j}\right) \in R^{n} \times T$. No caso de função covariância estacionária espaçotemporal a condição de inteiramente simétrica é ilustrada na Figura 12 e segue a equação 


$$
\begin{gathered}
C(h, u)=C(h,-u)=C(-h, u)=C(-h,-u), \\
\forall(h, u) \in R^{n} \times T
\end{gathered}
$$
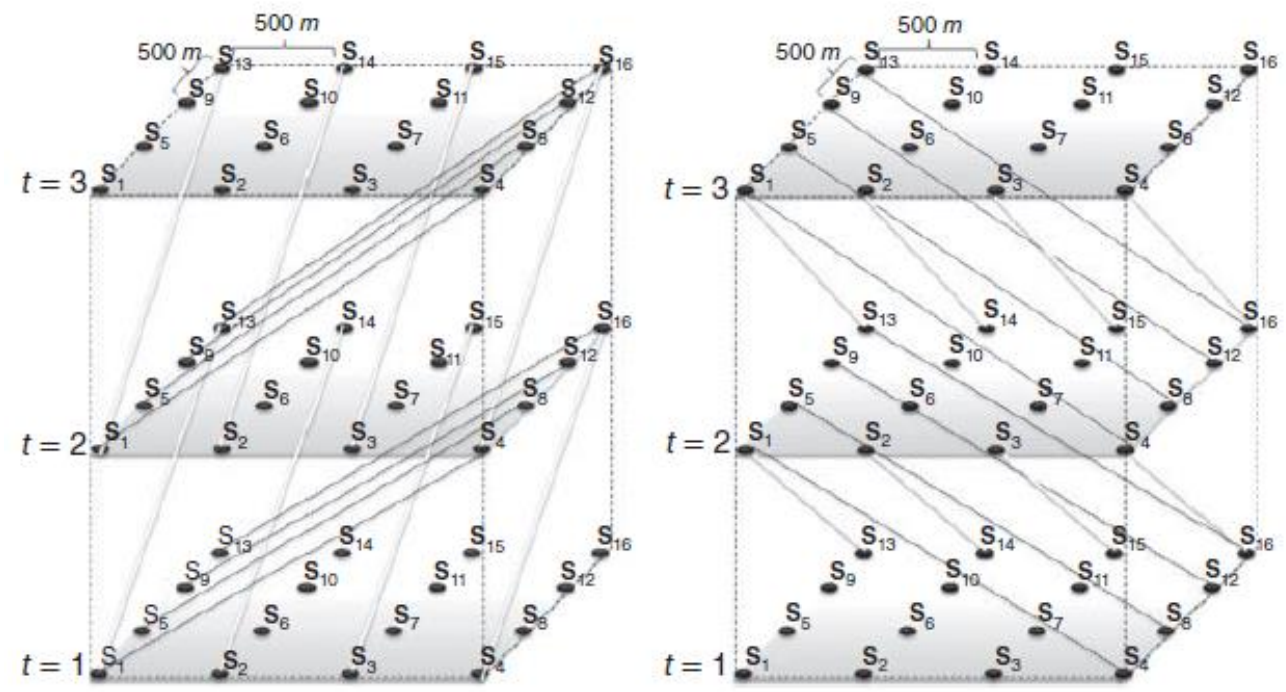

Figura 12 - Exemplo de covariância inteiramente simétrica (Montero et al., 2015)

(f) Função covariância com suporte compacto (Montero et al., 2015): quando a distância espacial ou temporal é suficientemente grande para quaisquer pares locais espaçotemporais $\left(\mathrm{s}_{1}, \mathrm{t}_{1}\right)$ e $\left(\mathrm{s}_{2}, \mathrm{t}_{2}\right) \in \mathrm{R}^{\mathrm{n}} \mathrm{x} \mathrm{T}$ que a função covariância $\mathrm{C}\left(\left(\mathrm{s}_{1}, \mathrm{t}_{1}\right),\left(\mathrm{s}_{2}\right.\right.$, $\left.\mathrm{t}_{2}\right)$ ) tende a zero.

(g) Função semivariograma espaçotemporal (Montero et al., 2015):

$$
\gamma\left(\left(s_{i}, t_{i}\right),\left(s_{j}, t_{j}\right)\right)=1 / 2 E\left(Z\left(s_{i}, t_{i}\right)-Z\left(s_{j}, t_{j}\right)\right)^{2}
$$

no caso de uma função aleatória com média constante, quando é possível definir uma função covariância e o semivariograma, estes são relacionados pela expressão média

$$
\begin{aligned}
& \gamma\left(\left(s_{i}, t_{i}\right),\left(s_{j}, t_{j}\right)\right)=1 / 2 \operatorname{Var}\left(Z\left(s_{i}, t_{i}\right)\right) \\
& +1 / 2 \operatorname{Var}\left(Z\left(s_{j}, t_{j}\right)\right)-C\left(\left(s_{i}, t_{i}\right),\left(s_{j}, t_{i}\right)\right)
\end{aligned}
$$


(h) Semivariograma intrinsecamente estacionário (Montero et al., 2015): se for intrinsecamente estacionário no espaço e no tempo é expresso por: $\gamma\left(\left(\mathrm{s}_{\mathrm{i}}, \mathrm{t}_{\mathrm{i}}\right),\left(\mathrm{s}_{\mathrm{j}}, \mathrm{t}_{\mathrm{j}}\right)\right)$ $=\gamma(\mathrm{h}, \mathrm{u}), \mathrm{h}=\mathrm{s}_{\mathrm{i}}-\mathrm{s}_{\mathrm{j}} \mathrm{e} \mathrm{u}=\mathrm{t}_{\mathrm{i}}-\mathrm{t}_{\mathrm{j}}$ representando o espaço e o tempo, respectivamente. As restrições $\gamma(., \mathrm{u})$ e $\gamma(\mathrm{h}$, .) são denominados, respectivamente, de semivariogramas puramente espacial e puramente temporal.

A Figura 13 ilustra a relação entre as principais classes das funções de covariância espaçotemporal encontradas. Se for possível encontrar qual categoria representa os dados, o modelo da função covariância será melhor ajustado. Gneiting et al. (2007) comentam que a maior classe é de estacionariedade ou não estacionariedade das funções de covariância, uma covariância separável pode ser estacionária ou não estacionária e, semelhantemente, para a classe de covariâncias inteiramente simétricas. Entretanto, uma função de covariância separável é sempre inteiramente simétrica, mas o inverso não é válido e isto tem implicações nos testes e no ajuste do variograma. Em particular, para rejeitar a separabilidade é necessário rejeitar a simetria. Li et al. (2007) e Cappello et al. (2018) discorrem sobre as propriedades de inteira simetria e separabilidade e mostram as suas hipóteses nulas, respectivamente, $H_{0}^{1}$ e $H_{0}^{2}$, então

$$
\begin{array}{ll}
H_{0}^{1}=\mathrm{C}(\mathrm{h}, \mathrm{u})-\mathrm{C}(\mathrm{h},-\mathrm{u})=0 & (\mathrm{~h}, \mathrm{u}) \in \Lambda \\
H_{0}^{2}=\frac{C(h, u)}{C(h, 0)}-\frac{C(0, u)}{C(0,0)}=0 & (\mathrm{~h}, \mathrm{u}) \in \Lambda
\end{array}
$$

Além disso, Sherman (2011) e De Iaco et al. (2016) explicam que entre as funções de covariância inteiramente simétricas, a função covariância é separável quando a função espaçotemporal puder ser fatorada (separada) no produto da função puramente espacial com a função puramente temporal $\left(\mathrm{C}(\mathrm{h}, \mathrm{u}) \sim \mathrm{C}_{\mathrm{s}}(\mathrm{h}) \mathrm{C}_{\mathrm{t}}(\mathrm{u})\right)$. Ou seja, para uma função ser separável, ela deve ser inteiramente simétrica. 
Classe geral das funções de covariância espaçotemporais

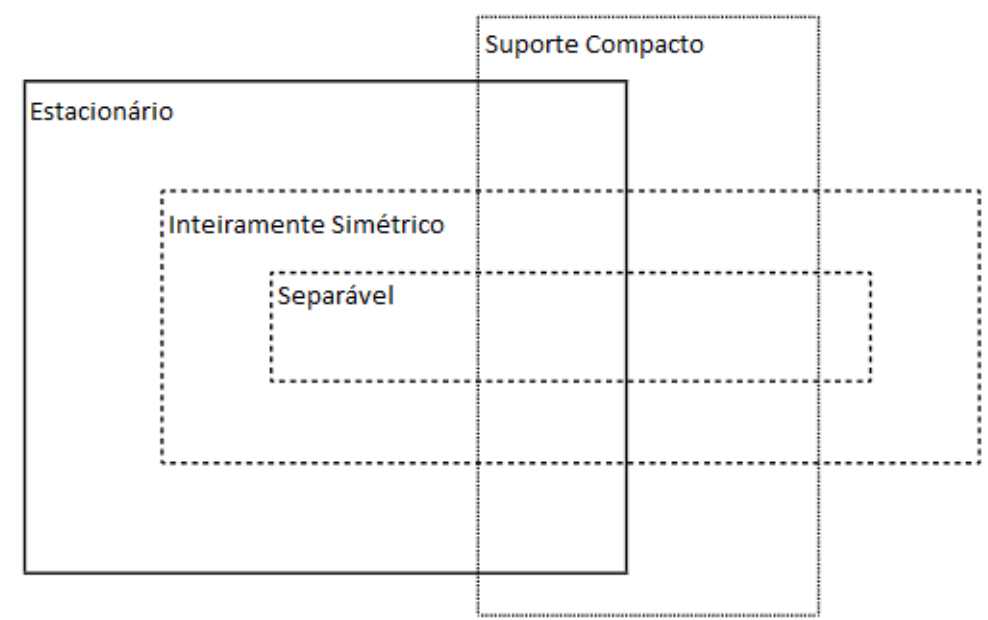

Figura 13 - Representação esquemática da relação entre separabilidade, inteira assimetria, estacionariedade, e suporte compacto (traduzida de Gneiting et al., 2007)

Então o variograma espaçotemporal é calculado com base em uma distância entre amostras, tanto no espaço (h) quanto no tempo (u). A Figura 14 expõe como esta distância relativa entre o espaço e o tempo pode ser contabilizada com um exemplo de pontos separados por uma distância no espaço de 1500 metros e no tempo de 2 unidades.

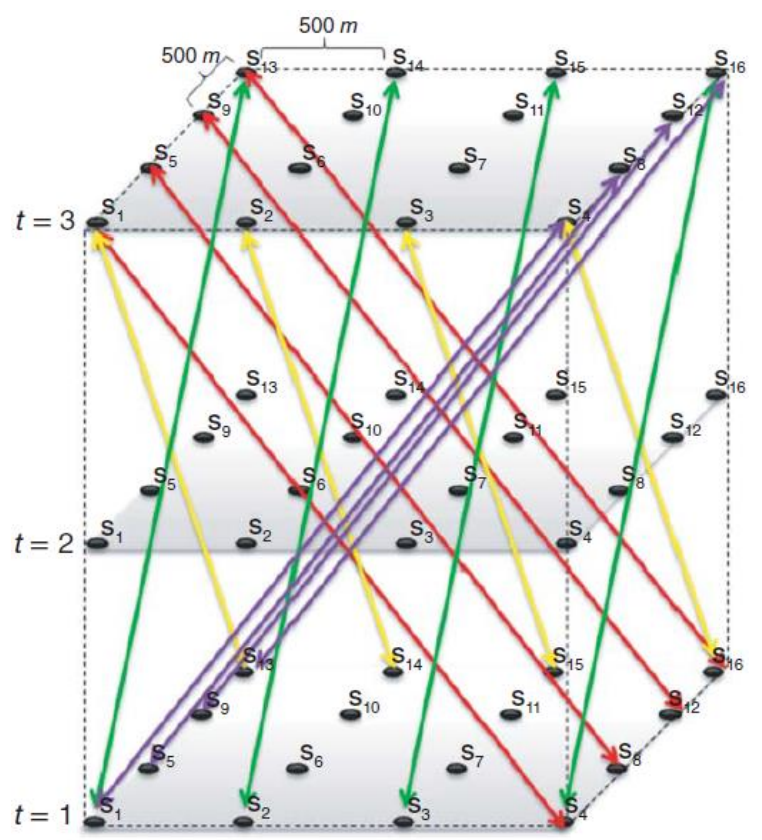

Figura 14 - Exemplo de pares de pontos separados por $\mathrm{h}=1500 \mathrm{~m} \mathrm{e} \mathrm{u}=2$ (Montero et al., 2015) 
Após a covariância espaçotemporal ser calculada para todos os pares de pontos, inclusive os pares somente no espaço e os pares somente no tempo, podemos criar um variograma como um gráfico de 3 eixos, em que usualmente se coloca a covariância (ou variância) espacial no eixo z, os valores nos passos do espaço em x e os valores dos passos no tempo em y. O variograma espaçotemporal experimental típico pode ser visto na Figura 15.

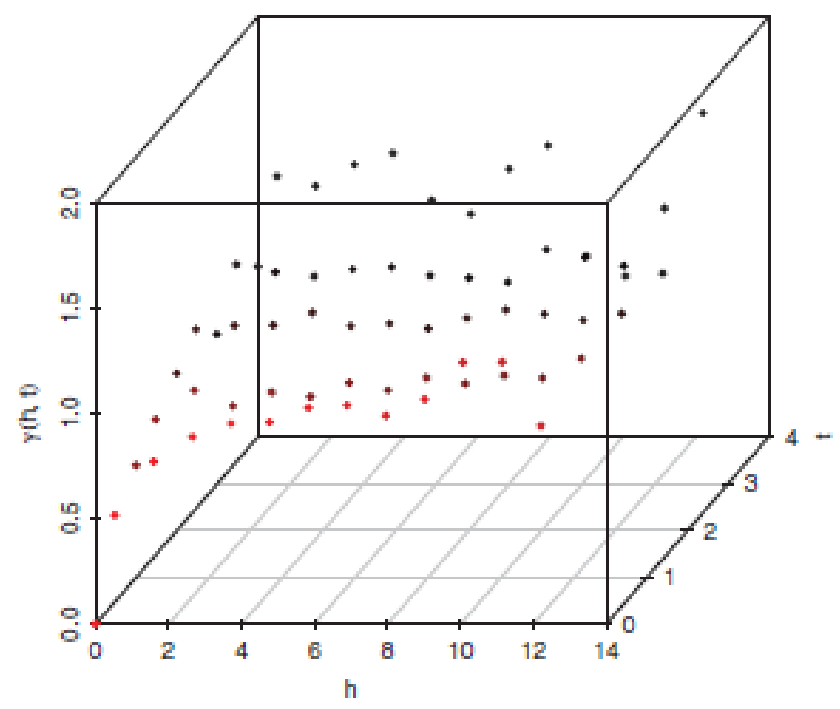

Figura 15 - Exemplo de variograma espaçotemporal (Montero et al. 2015)

\subsubsection{Modelos de covariância espaçotemporal}

Segundo De Iaco et al. (2016), a análise da correlação espaçotemporal pode ser abordada para fenômenos naturais como um modelo de covariância derivado dos dados disponíveis. Em outras palavras, pode-se ajustar um modelo de covariância a partir de uma superfície empírica como um variograma (Figura 15). A classe de funções espaço-tempo separáveis faz sentido para funções covariância espaçotemporais, porém para o caso do semivariograma não, pois o produto de semivariogramas não garante necessariamente um semivariograma válido. Montero et al. (2015) dizem que quando se trabalha com funções aleatórias espaçotemporais, a função covariância é mais adequada, de qualquer modo a função covariância pode ser facilmente transformada na função variância e vice-versa. Sherman (2011) demonstra que, de maneira similar à geoestatística espacial, pode-se escrever a função 
covariância com relação à função variância como $\gamma(\mathrm{h}, \mathrm{u})=\mathrm{C}(0,0)-\mathrm{C}(\mathrm{h}, \mathrm{u})$ para processos espaçotemporais com estacionariedade de segunda ordem.

Montero et al. (2015) generalizam algumas propriedades da função covariância espacial para o caso espaçotemporal e considerando as funções no $\mathrm{R}^{\mathrm{n}} \mathrm{x} \mathrm{T} \equiv \mathrm{R}^{\mathrm{n}+1}$, tem-se que as equações (Eq. 29), (Eq. 30) e (Eq. 31) são funções covariância que também são definidas em $\mathrm{R}^{\mathrm{n}} \mathrm{x}$ T:

$$
\begin{array}{ll}
\mathrm{C}(\mathrm{h}, \mathrm{u})=\mathrm{bC}_{1}(\mathrm{~h}, \mathrm{u}) & \forall \mathrm{b}>0 \\
\mathrm{C}(\mathrm{h}, \mathrm{u})=\mathrm{C}_{1}(\mathrm{~h}, \mathrm{u})+\mathrm{C}_{2}(\mathrm{~h}, \mathrm{u}) & \\
\mathrm{C}(\mathrm{h}, \mathrm{u})=\mathrm{C}_{1}(\mathrm{~h}, \mathrm{u}) \mathrm{C}_{2}(\mathrm{~h}, \mathrm{u}) &
\end{array}
$$

Além das funções supracitadas, Montero et al. (2015) demonstram diversas funções que preservam a covariância definidas em $\mathrm{R}^{\mathrm{n}} \mathrm{x} \mathrm{T}$, pelas características referentes a propriedades de estabilidade, de permissividade nos limites das funções, de vetores aleatórios e funções como de densidade espectral com transformações de Fourier e de covariância inteiramente simétricas entre outras. Todas estas funções podem ser utilizadas para modelar a covariância espaçotemporal ( $\mathrm{R}^{\mathrm{n}} \mathrm{x}$ T), porém as equações (Eq. 29), (Eq. 30) e (Eq. 31) são as mais comuns devido sua simplicidade matemática e fácil aplicação.

De Iaco et al. (2013b) e De Iaco et al. (2016) mostram que na modelagem de funções de covariância espaçotemporais, deve-se garantir que o modelo seja definido positivo e, idealmente, deve-se conhecer as características da superfície de covariância espaçotemporal empírica, pois elas podem ajudar a selecionar adequadamente uma função de covariância que melhor se ajuste. Assim, deve-se conhecer características como comportamento na origem e no infinito, separabilidade, comportamento assintótico, anisotropia e inteiramente simétrico. Desta maneira, diversos modelos foram criados e cada um aborda classes das funções espaçotemporais diferentes. Assim, o grupo de modelos separáveis apresenta interações mais limitadas, porém ele é a base para modelos não-separáveis com processos misturados (Ma, 2002). Montero et al. (2015) discorrem que ajustar um modelo de semivariograma ou de covariância pode ser feito manualmente, utilizando processos automatizados ou ambos. O que importa é que o modelo escolhido no ajuste, capture o tipo de continuidade assumido pela variável regionalizada e suas hipóteses de estacionariedade e separabilidade associadas. Além 
disso, o conhecimento do fenômeno em estudo ajuda no processo de ajuste. Os principais modelos de covariância espaçotemporal são:

(a) Modelo Métrico (Dimitrakopoulos e Luo, 1994):

$$
\begin{array}{cc}
C(h, u)=C\left(|| h||^{2}+c|u|^{2}\right), & (h, u) \in \\
R^{n} \times T, c>0 & (h, u) \in \\
\gamma(h, u)=\gamma\left(\sqrt{c_{1}^{2}|| h \|^{2}+c_{2}^{2}|u|^{2}}\right) & (h, u) \in \\
R^{\mathrm{n}} \times \mathrm{T}, \mathrm{c}_{1}, \mathrm{c}_{2}>0 & \\
\gamma(\mathrm{h}, \mathrm{u})=\mathrm{C}(0,0)-\mathrm{C}\left(\sqrt{\left.c_{1}^{2}|| h\left|\|^{2}+c_{2}^{2}\right| u\right|^{2}}\right) & \\
(\mathrm{h}, \mathrm{u}) \in \mathrm{R}^{\mathrm{n}} \times \mathrm{T}, \mathrm{c}_{1}, \mathrm{c}_{2}>0 &
\end{array}
$$

onde c é uma constante positiva e $\|\mathrm{h}\|+\mathrm{c}|\mathrm{u}|$ é uma distância em $\mathrm{R}^{\mathrm{n}} \times \mathrm{T}$ e $\mathrm{C}(0,0)$ é a variância espaçotemporal. A Figura 16 mostra uma representação do modelo métrico como uma distância euclidiana em que a distância espaçotemporal m é calculada pela hipotenusa dos catetos u (distância temporal) e h (distância espacial).

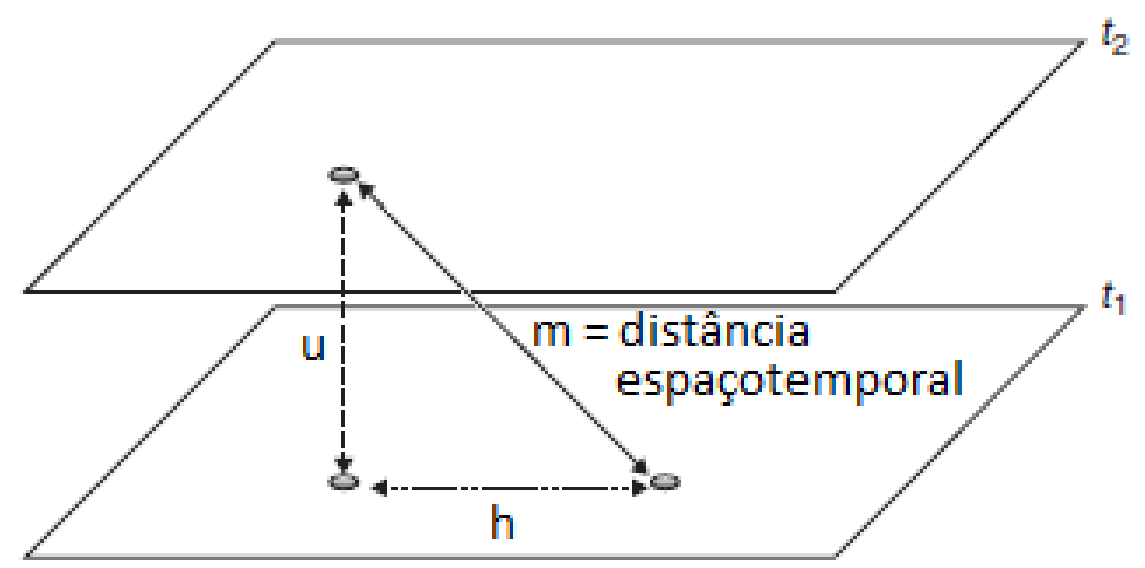

Figura 16 - Representação da distância espaçotemporal do modelo métrico (traduzida de Montero et al. 2015) 
(b) Modelo Soma (Rouhani e Hall, 1989):

$$
\begin{array}{ll}
C(h, u)=C_{s}(h)+C_{t}(u), & (h, u) \in R^{n} \times T \\
\gamma_{s t}(h, u)=\gamma_{s}(h)+\gamma_{t}(u), & (h, u) \in R^{n} \times T
\end{array}
$$

com $C_{s}$ e $\gamma_{s}$ sendo a covariância espacial e a semivariância espacial e $C_{t}$ e $\gamma_{t}$ a covariância temporal e a semivariância temporal, respectivamente.

(c) Modelo Métrico-Soma (Bilonick, 1988 e Snepvangers et al., 2003):

$$
\begin{gathered}
C_{s m}(h, u)=C_{s}(h)+C_{t}(u)+C_{\text {joint }}\left(\sqrt{\left.|| h\right|^{2}+c|u|^{2}}\right), \\
(h, u) \in R^{n} \times T \\
\gamma_{s t}(h, u)=\gamma_{s}(h)+\gamma_{t}(u)+\gamma\left(\sqrt{\|h\|^{2}+c|u|^{2}}\right), \\
(h, u) \in R^{n} \times T
\end{gathered}
$$

onde c é uma constante positiva e $\|\mathrm{h}\|+\mathrm{c}|\mathrm{u}|$ é uma distância em $\mathrm{R}^{\mathrm{n}} \times \mathrm{T}$.

(d) Modelo Produto (De Cesare et al. 1997):

$$
\begin{aligned}
& C(h, u)=C_{s}(h) C_{t}(u), \quad(h, u) \in R^{n} x T \\
& \gamma(h, u)=C_{s}(0) \gamma_{t}(u)+C_{t}(0) \gamma_{s}(h)-\gamma_{s}(h) \gamma_{t}(u) \\
& (h, u) \in R^{n} \times T
\end{aligned}
$$

(e) Modelo Produto-Soma (De Cesare et al., 2001a,b e De Iaco et al., 2001):

$$
\begin{gathered}
C(h, u)=k_{s}(h) C_{t}(u)+C_{s}(h)+C_{t}(u), \\
(h, u) \in R^{n} \times T
\end{gathered}
$$




$$
\begin{gathered}
\gamma(h, u)=\gamma_{s}(h)+\gamma_{t}(u)-k \gamma_{s}(h) \gamma_{t}(u) \\
(h, u) \in R^{n} \times T
\end{gathered}
$$

com $\mathrm{k}=\frac{C_{s}(0)+C_{t}(0)-C(0,0)}{C_{s}(0) C_{t}(0)}, \mathrm{C}(0,0)$ é o patamar de $\gamma, \mathrm{C}_{\mathrm{s}}(0)$ é o patamar de $\gamma_{\mathrm{s}}$ e $\mathrm{C}_{\mathrm{t}}(0)$ é o patamar de $\gamma_{\mathrm{t}}$.

Além destes modelos, existem diversos outros que modelam a covariância espaçotemporal com diferentes funções, como: produto integrado (De Iaco, 2002), baseado em misturas, função cópula e função completamente monótona (Porcu e Mateu, 2007), função completamente monótona (Gneiting, 2002), misturas de escalas (Ma, 2002 e Ma, 2003a), função distribuição espectral da covariância (Cressie e Huang, 1999), função densidade espectral (Stein, 2005) funções de média quase-aritméticas (Porcu et al., 2009) e equações diferenciais parciais estocásticas (Hristopulos e Tsantili, 2017). Além dos modelos baseados em funções, ainda existem modelos específicos para dados não estacionários (Ma, 2003b e Porcu e Mateu, 2007), para capturar e estudar a anisotropia (Porcu et al., 2006) e para dados não inteiramente simétricos (Gneiting et al., 2007), pois existem regimes naturais como vento, correntes oceânicas que comprometem a simetria.

\subsubsection{Krigagem espaçotemporal}

Uma função covariância espaçotemporal permite a estimativa por krigagem em qualquer momento t no tempo e qualquer posição s no espaço (Kyriakidis e Journel, 1999). Sherman (2011) discorre que para utilizar modelos de covariância na estimativa, deve-se considerar modelos conhecidos, pois qualquer função de covariância precisa ser definida nãonegativa e, em geral, os estimadores de momento das funções de covariância não satisfazem essas propriedades. Além disso, para obter uma previsão ideal, $\operatorname{Cov}\left[Z\left(s_{0}, t_{0}\right), Z_{n, \mathrm{~T}}\right]$ é mandatório, ou seja, a covariância a qualquer distância espaçotemporal deve ser conhecida. O mesmo autor ainda explica que uma combinação linear (com coeficientes positivos) de funções de covariância válidas são funções válidas. Ou seja, o produto de duas funções válidas é válido.

De Cesare et al. (1997) explicam que considerar o tempo como outra dimensão não altera o estimador da krigagem e nem suas equações. Além disso, Cressie e Wikle (2011) destacam que a caracterização da dependência espacial e temporal (covariância 
espaçotemporal) pode ser utilizada para a krigagem. Desta forma, se $(s, t)$ é uma localização sem amostragem no espaço-tempo e com a distribuição $\left(Z\left(s_{1}, t_{i}\right), \ldots, Z\left(s_{N}, t_{i}\right), i=1, \ldots, p\right)$ então $\mathrm{Z}(\mathrm{s}, \mathrm{t})$ pode ser estimada por

$$
\hat{Z}(s, t)=\sum_{i=1}^{m} \sum_{j=1}^{n_{i}} \lambda_{i, j} Z\left(s_{i, t}, t_{i}\right)
$$

onde $\lambda_{n, i}$ são os pesos da krigagem e não há suposições sobre quaisquer interrelações entre o espaço e o tempo em um ponto. $\mathrm{O}$ estimador interpolará no espaço e/ou no tempo e extrapolará no espaço e/ou no tempo. A variável $Z(s, t)$ pode ser estimada/simulada em todos os pontos do domínio e em qualquer período temporal desejado com a geoestatística espaçotemporal. A variável $Z(s, t)$ pode ser estimada/simulada em todos os pontos do domínio e em qualquer período temporal desejado com a geoestatística espaçotemporal.

\subsection{Séries Temporais}

Cowpertwait e Metcalfe (2009) ilustram que em diversas linhas da ciência, engenharia e comércio, existem variáveis medidas sequencialmente no tempo. Por exemplo, taxas bancárias medidas diariamente, temperaturas medidas diversas vezes ao dia em muitas cidades, a quantidade de chuva medida em diversos lugares, o crescimento anual de países ou regiões calculadas por seus departamentos de estatística. Quando estas e outras variáveis são medidas no tempo a um intervalo fixo, o resultado é uma série temporal. Shumway e Stoffer (2011) discorrem que o objetivo principal da análise de séries temporais é desenvolver modelos matemáticos que resultem em descrições plausíveis dos dados. Para fornecer ferramentas estatísticas que descrevem o caráter dos dados que flutuam de modo aleatório no tempo, assume-se que a série pode ser definida como uma coleção de variáveis aleatórias indexadas de acordo com o tempo. Além disso, a abordagem do domínio do tempo é motivada pela presunção de que as correlações entre pontos adjacentes no tempo explicam, em termos, a dependência dos valores atuais aos passados. 
Jenkins e Watts (1968) descrevem série temporal como uma função aleatória ou determinística $\beta$ da variável $\mathrm{t}$, usualmente $\beta(\mathrm{t})$ é função do tempo, mas pode ser de outra propriedade física como o espaço. Entre as características das séries temporais, elas não podem prever exatamente seu comportamento futuro, como no caso de uma função determinística do tempo. Em algumas áreas da matemática aplicada assume-se que processos físicos podem ser descritos por funções do tempo (determinísticas), entretanto inspeções criteriosas da amplitude revelam flutuações com o tempo. É necessário descrever as séries temporais por leis probabilísticas ou modelos desde que diferentes séries se assemelhem em suas proporções médias. De acordo com Woodward et al. (2011), processo estocástico $\{\beta(\mathrm{t}) ; \mathrm{t} \in \mathrm{T}\}$ é um conjunto de variáveis aleatórias, onde $\mathrm{T}$ representa o tempo, que se refere ao processo estocástico como uma série temporal. Qualquer valor observado da variável aleatória é um número real para $\omega \in \Omega$. Qualquer realização da série temporal $\{\beta(\mathrm{t}) ; \mathrm{t} \in \mathrm{T}\}$ é um conjunto de valores reais $\{\beta(\mathrm{t}, \omega) ; \mathrm{t} \in \mathrm{T}\}$ para dado valor fixo de $\omega \in \Omega$, ou seja, a realização da série temporal é o conjunto de valores que resulta da ocorrência de um evento observado. No estudo de séries temporais, é comum calcular uma única realização, que é como analisar as propriedades de uma variável aleatória com apenas uma observação. Woodward et al. (2011) ainda explicam que os conceitos de estacionariedade e ergodicidade apresentam papel importante para a habilidade de analisar séries temporais de maneira efetiva. Determinado processo é dito estacionário no tempo se estiver no estado de "equilíbrio estatístico", ou seja, que seu comportamento não mude com o tempo e processo ergódigo é aquele para o qual as médias de conjuntos podem ser consistentemente estimadas a partir de uma única realização.

Montgomery et al. (2015) definem que os passos básicos da modelagem e previsão de séries temporais são:

(a) Plotar a série temporal e determinar suas principais características, se há tendências e sazonalidades. Procurar por outliers ou indicadores de que a série muda de comportamento.

(b) Eliminar qualquer componente de tendência ou sazonalidade, por diferenciação ou ajustando um modelo apropriado aos dados e considerar utilizar dados transformados. O objetivo é produzir resíduos estacionários de segunda ordem.

(c) Desenvolver o modelo de previsão para os resíduos. Não é incomum descobrir que há vários modelos plausíveis e análises adicionais podem ser feitas para determinar o melhor. 
(d) Validar o desempenho do modelo. Pode envolver separação de algumas amostras e validação cruzada. O objetivo é selecionar o melhor modelo para fazer as previsões.

(e) Devolver os valores futuros para a escala original. É interessante a comparação da série original com valores previstos na escala original, para isso, deve-se reverter as transformações e qualquer diferenciação feita para remover tendências e sazonalidade.

(f) Desenvolver e implementar um procedimento de monitoramento e previsão para garantir que a deterioração do desempenho seja notada rapidamente.

Prado e West (2010) comentam que inicialmente deve-se realizar a análise estatística que consiste em fazer um estudo descritivo dos dados para resumir suas principais características. Além disso, uma das técnicas mais utilizadas em séries temporais é explorar os padrões de correlação mostrados nas séries, ou diversas séries, em pontos no tempo. Isto é feito plotando os valores de autocorrelação, que são estimados das funções de autocorrelação (ACF). Considerando $\{\beta(t), \mathrm{t} \in \mathrm{T}\}$ como uma série temporal, a autocovariância de um processo estacionário para todo t e a função autocovariância depende apenas do passo temporal $u$ (passo temporal é a diferença (ou "distância") temporal entre valores estudados, sendo semelhante ao passo estudado na geoestatística)

$$
\mathrm{C}(\mathrm{u})=\operatorname{Cov}\{\mathrm{yt}, \mathrm{yt}-\mathrm{u}\}
$$

a ACF é dada por

$$
\rho(\mathrm{u})=\frac{\mathrm{C}(\mathrm{u})}{\mathrm{C}(0)}
$$

Shumway e Stoffer (2011) explicam que a ACF mede a dependência linear entre dois pontos na mesma série observada em tempos diferentes. Séries suaves mostram altas correlações mesmo quando o passo temporal é grande. Enquanto séries erráticas tendem apresentar correlações próximas a zero em passos temporais grandes. A Figura 17 ilustra um exemplo de ACF. 


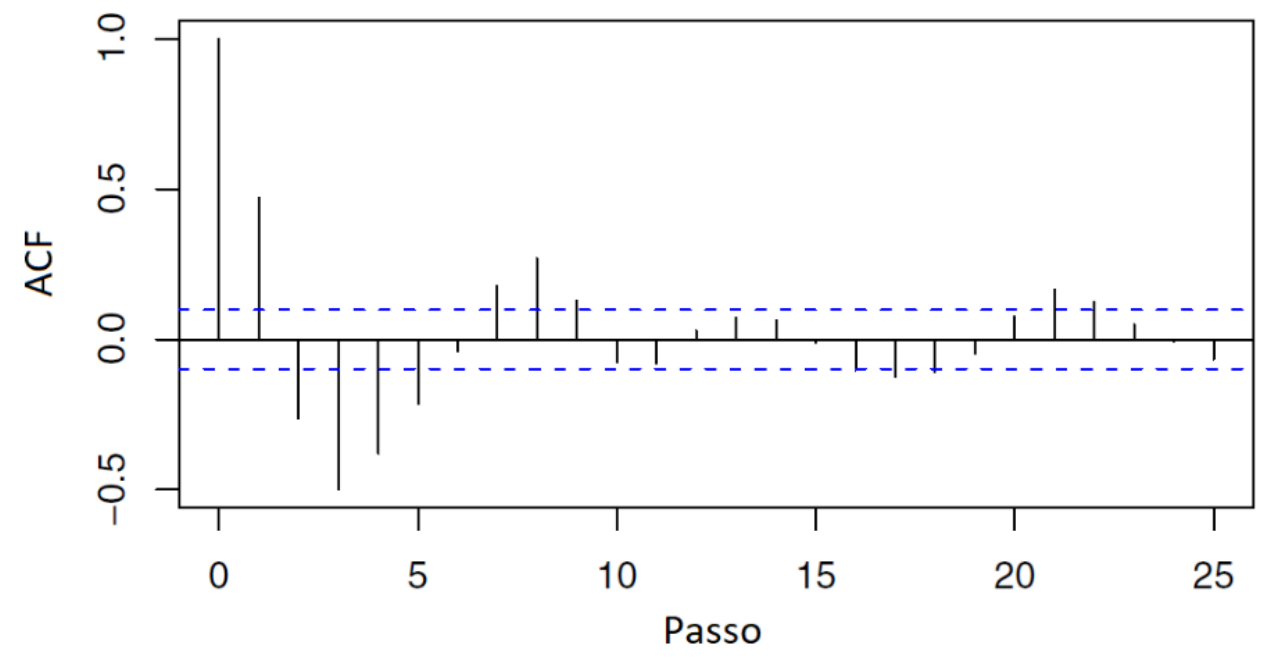

Figura 17 - Exemplo de uma função de autocorrelação (Cowpertwait e Metcalfe, 2009)

De modo geral, é necessário que uma série temporal seja estacionária e talvez a forma mais fácil de modelar a não-estacionariedade é assumi-la como tendência, ou seja, onde o processo tem estacionariedade ao redor de uma tendência (Shumway e Stoffer, 2011). Desta maneira, Cowpertwait e Metcalfe (2009) realçam que uma vez que os efeitos da sazonalidade e da tendência são identificados, pode-se remover estes componentes. Se utilizar uma decomposição aditiva, deve-se calcular as séries temporais ajustadas sazonalmente e remover a tendência por subtração, restando apenas a componente aleatória. Em muitos casos, variáveis consecutivas são correlatas e se for possível identificar estas correlações, pode-se melhorar a previsão. Tendências nas séries temporais podem ser classificadas tanto como estocásticas quanto como determinísticas. Pode-se classificar a tendência como estocástica quando ela mostra mudanças inexplicáveis. Por outro lado, quando se tem explicações físicas plausíveis para as tendências, elas devem ser modeladas preferencialmente de maneira determinística. As tendências e sazonalidades determinísticas podem ser modeladas usando regressão. Segundo Brockwell e Davis (2010), a seleção de modelos probabilísticos é parte importante do estudo. Diversas séries temporais apresentam clara tendência nos dados. Além disso, muitas séries também são influenciadas por fatores sazonais que podem ser modelados com uma componente de período fixo. Para representar esta sazonalidade, assumindo que não há tendência nos dados, um modelo simples é a regressão harmônica. Então, o modelo de decomposição clássico, como descrito em Brockwell e Davis (2010) é 


$$
\beta(t)=\operatorname{tr}(t)+\operatorname{sea}(t)+\operatorname{res}(t)
$$

onde $\beta(t)$ é a série temporal, $\operatorname{tr}(t)$ é a função da componente de tendência, sea $(t)$ é a função periodicidade e $\operatorname{res}(t)$ é um ruído aleatório, sem tendência ou periodicidade cujas observações são variáveis aleatórias independentes e que apresenta média zero. Uma técnica para estimar $\operatorname{tr}(t)$ é a regressão por mínimos quadrados. Shumway e Stoffer (2011) mostram que a regressão linear no contexto das séries temporais considera que uma série dependente é afetada por um conjunto de séries conhecidas e pode-se utilizar regressão linear simples para estimar a tendência com o ajuste do modelo:

$$
\operatorname{tr}(t)=b_{1}+b_{2} t+\varepsilon(t)
$$

Onde $b_{1}$ e $b_{2}$ são os coeficientes desconhecidos da regressão (intercepto e coeficiente angular, respectivamente) e $\varepsilon(t)$ é um erro aleatório (ruído). Enquanto isso, sea $(t)$ pode ser modelada como harmônicos da série de Fourier. De acordo com Bloomfield (1976), a análise de Fourier ou análise harmônica de uma série temporal é a decomposição da série na soma de componentes determinísticos. Métodos de mínimos quadrados são muito utilizados para ajustar estes parâmetros aos dados observados, pois têm muitas vantagens teóricas e computacionais. O mesmo autor ainda mostra que a senóide de frequência $\omega$ (em radianos por unidade de tempo) pode ser escrita como uma função não linear tradicional e como uma função linear:

$$
\begin{gathered}
\operatorname{sea}(t)=R \cos (\omega t+\phi) \\
\operatorname{sea}(t)=\mu+A \cos \omega t+B \operatorname{sen} \omega t+\varepsilon(t)
\end{gathered}
$$

onde $\mathrm{R}$ é a amplitude, $\phi$ é a fase, $\mathrm{A}=\mathrm{R} \cos \phi, \mathrm{B}=-\mathrm{R} \sin \phi$ e $\varepsilon(t)$ é um erro aleatório. Se não houver outra tendência ou sazonalidade, este pode ser um ruído branco gaussiano e sua função autocorrelação é próxima a zero (Tsay, 2010). É aconselhável que a frequência $\omega$ seja 
conhecida e constante para melhorar o ajuste, uma vez que considerando A e B como parâmetros a função é linear se $\omega$ é fixo (Bloomfield, 1976).

Na Figura 18, mostra-se um exemplo de dados observados sequencialmente no tempo (A), como são os modelos de tendência (B), sazonalidade (C) e o resultado da remoção destas componentes como variações aleatórias ou resíduo (D).

Segundo Kedem e Fokianos (2002), na regressão linear ordinária, o problema é relacionar o resultado médio de uma variável de interesse para um conjunto de variáveis explanatórias pela média da equação linear. Em muitos casos, isto é feito assumindo que as variáveis são independentes e têm distribuição normal. Existem situações que a regressão linear ordinária gera algumas inconsistências com observações não normais e algumas são resolvidas por modelos lineares generalizados. Utilizando a verossimilhança parcial pode-se transportar as principais características de inferência de dados independentes para dados de séries temporais. Na prática, muitas vezes, a natureza da dependência não é conhecida e, por isso, a verossimilhança não é facilmente atingida. A verossimilhança é definida como distribuição conjunta de variáveis como funções de parâmetros desconhecidos.

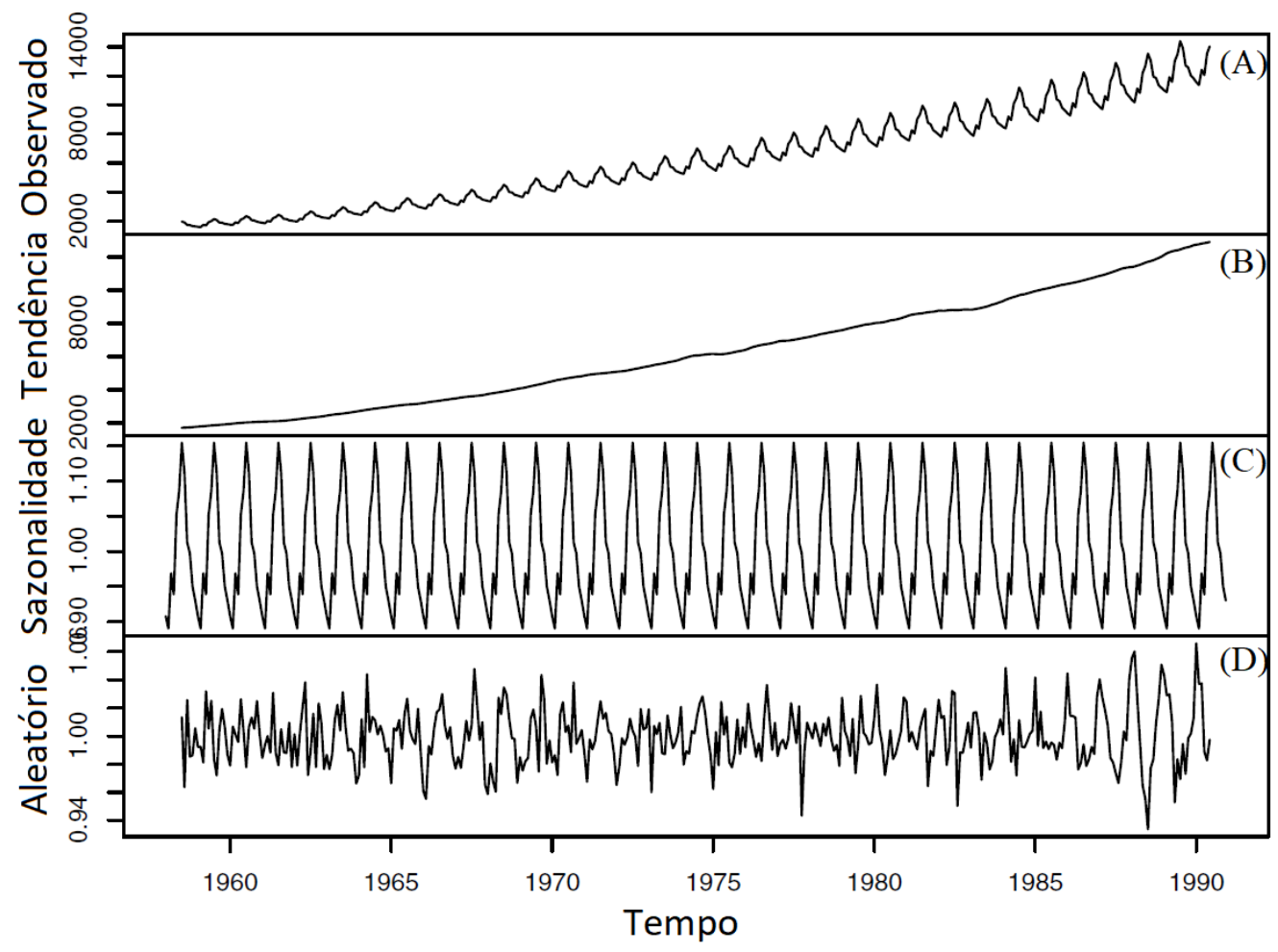

Figura 18 - Demonstração da modelagem de uma série temporal por sua tendência, sazonalidade e sua decomposição em variações aleatórias (traduzida de Cowpertwait e Metcalfe, 2009) 
Shumway e Stoffer (2011) esclarecem que a regressão clássica pode não ser suficiente para explicar toda a dinâmica das séries temporais. Prado e West (2010) expõem que modelos de séries temporais autoregressivos são centrais para a análise de dados temporais estacionários. Box et al. (2015) explicam que a descrição dos processos estocásticos das séries temporais deve ser flexível para modelar a maioria das situações. Assim, os modelos autoregressivos (AR) são modelos estocásticos extremamente úteis na representação de séries em andamento. Nesse tipo de modelo, o valor contínuo do processo é expresso como um agregado linear finito de valores anteriores do processo e uma componente aleatória $\varepsilon(t)$. Então um processo autorregresivo $(\mathrm{AR}(\mathrm{p}))$ com $\mathrm{p}$ componentes da série $\beta(t)$ pode ser apresentado como (Box et al., 2015):

$$
\Phi(\mathrm{B}) \beta(t)=\varepsilon(t)
$$

onde B é o operador de defasagem do operador $\Phi$ do $\mathrm{AR}$ e $\varepsilon(t)$ é o ruído branco.

Os mesmos autores ainda citam que outra maneira prática de representar observações de séries temporais é o processo de médias móveis (MA(q)). Este modelo considera que a série $\beta(t)$ é linearmente dependente de um número finito q de componentes. Este modelo geralmente é escrito como (Box et al., 2015):

$$
\beta(t)=\theta(\mathrm{B}) \varepsilon(t)
$$

Ambos os métodos (autoregressivo e médias móveis) são definidos por um operador de defasagem B. Misturando os processos AR e MA nos leva a um modelo flexível que ajusta quase qualquer série temporal. Entretanto os 2 métodos exigem estacionariedade (Box et al., 2015). Assim, Box e Jenkins (1970) definiram a forma geral do ARIMA (processos autoregressivo integrado com médias móveis) como:

$$
\Phi(\mathrm{B}) \cdot(1-\mathrm{B})^{\mathrm{d}} \cdot \beta(t)=\theta_{0}+\theta(\mathrm{B}) \cdot \varepsilon t
$$


Então o modelo ARIMA (p, d, q) é definido por p como número de parâmetros do operador $\Phi$ do AR, d é o grau de diferenciação e q é o número de parâmetros do operador $\theta$ da MA. O modelo é muito generalista e considera os casos em que apenas um dos processos é necessário ou o caso em que é necessário misturar todos eles (Box et al., 2015).

Todos os detalhes, aplicações e provas matemáticas deste modelo podem ser encontrados em: Box e Jenkins (1970); Newbold et al. (1993); Janacek (2001); Chatfield (2003); Cowpertwait e Metcalfe (2009); Prado e West (2010); Brockwell e Davis (2010); Woodward et al. (2011), Box et al. (2015) e outros.

Para avaliar e escolher o melhor modelo, existem alguns procedimentos propostos para analisar o número de parâmetros e sua verossimilhança, o método mais utilizado para avaliar o ARIMA é o critério de informação de Akaike (AIC), que foi descrito por Akaike (1974) como

$$
\mathrm{AIC}=-2 \log (\mathrm{L})+2 \mathrm{k}
$$

Onde L é a máxima verossimilhança e $\mathrm{k}$ é o número de parâmetros ajustados independentemente do modelo.

Woodward et al. (2011) explicam que existem problemas na utilização do AIC e que o principal problema é a sua tendência de superestimar as ordens dos modelos conforme as realizações são maiores. Diversos pesquisadores já propuseram ajustes ao AIC para resolver seus problemas e as alternativas mais utilizadas são critério bayesiano de informação (Schwarz, 1978), critério de informação de Hannan-Quinn (Hannan e Quinn, 1979) e critério de informação de Akaike corrigido (Hurvich e Tsai, 1989).

Cowpertwait e Metcalfe (2009) mostram que há séries que exibem períodos de crescimento da variabilidade (volatilidade) de maneira não regular, estas são chamadas de heteroscedásticas e se esta mudança na variância possuir relação com o tempo são chamadas de heteroscedásticas condicionais. Para trabalhar com a volatilidade, pode-se utilizar um modelo autoregressivo condicional heteroscedástico generalizado $(\mathrm{GARCH}(\mathrm{q}, \mathrm{p}))$.

Para nomear poucos exemplos dos principais métodos e possíveis aplicações das pesquisas mais recentes sobre séries temporais, alguns trabalhos são mencionados a seguir. 
Koutroumanidis et al. (2006) aplicaram o modelo ARIMA e lógica difusa (fuzzy) para auxiliar um sistema de suporte de decisões que modelam e preveem séries temporais de pescaria. Kärner (2009) utilizou o tipo de modelo ARIMA para analisar que a série temporal da irradiância solar no topo da atmosfera é similar a série temporal da temperatura do ar. Considerando a sazonalidade, D’Urso et al. (2017) estudaram um grupo de séries temporais com padrões sazonais semelhantes usando análise de valor extremo com agrupamento difuso (fuzzy clustering) para séries temporais ao nível do mar e Herrera et al. (2018) analisaram os eventos climáticos extremos (ondas de calor) utilizando um conjunto de regressão quantílica (quantile regression ensemble) para combinar temperaturas extremas observadas ou simuladas. De maneira similar, Curceac et al. (2019) previram a temperatura do ar em Abu Dhabi com o SARIMA e a análise de dados funcionais não-paramétricos. Reisen et al. (2014) propuseram e aplicaram um modelo SARFIMA-GARCH e alcançaram bons resultados na previsão da poluição do ar (PM10) para modelos com memória sazonal longa e volatilidade. Chen e Boccelli (2018) desenvolveram um kit de ferramentas para séries temporais multi-sazonais. Maçaira et al. (2018) resumiram a revisão de literatura da análise de séries temporais com variáveis explicativas (variáveis exógenas). Além disso, Xu e Shen (2013) e Zhou et al. (2015) utilizaram análise harmônica para estimar dados ausentes das imagens da Land Surface Temperature (LST) obtidos do sensor MODIS no satélite TERRA. Utilizando a mesma fonte de imagens, Yoo et al. (2018) estimaram a temperatura máxima e mínima diária do ar utilizando variáveis auxiliares (elevação, radiação solar, NDVI, latitude, longitude, aspecto e porcentagem de área impermeável). Scheffel et al. (2018) utilizaram uma abordagem espectral de tempo para previsão numérica do tempo.

\subsection{Revisão Metodológica: Previsão Espaçotemporal}

Para criar mapas futuros a partir de alguns pontos com diferentes localizações e com amostragem de séries temporais, $\mathrm{Z}(\mathrm{s}, \mathrm{t})$, pode-se aplicar geoestatística espaçotemporal. Kyriakidis e Journel (1999) definem a variável aleatória espaçotemporal como uma variável que pode receber uma série de valores (realizações) em qualquer local no espaço s em um instante no tempo t. Os autores explicam que o domínio espaço-tempo deve ser considerado apenas como um sistema de coordenadas, onde as observações são marcadas por um vetor de coordenadas espaciais (eixos X e Y) de uma coordenada temporal t (eixo Z). A isotropia é bem explicada no espaço, no entanto, ela não tem significado em uma estrutura espaçotemporal 
devido à ordem intrínseca e à não reversibilidade do tempo. Além disso, unidades e escalas são distintas entre espaço e tempo, e também não podem ser comparadas no sentido físico (Kyriakidis e Journel, 1999). Da mesma forma, Montero et al. (2015) mostram que toda localização espaçotemporal é um ponto no $R^{n} x R$ sendo $R^{n}$ o espaço euclidiano com $n$ dimensões e $\mathrm{R}$ a dimensão tempo. Assim, do ponto de vista matemático $\mathrm{R}^{\mathrm{n}} \mathrm{x} \mathrm{R}=\mathrm{R}^{\mathrm{n}+1}$, porém não há sentido físico e social devido à diferença entre os eixos de coordenadas do sistema espaçotemporal.

As variáveis espaçotemporais $\mathrm{Z}(\mathrm{s}, \mathrm{t})$ são, normalmente, registradas em intervalos regulares em pontos fixos (Chilès e Delfiner, 1999), como, por exemplo, estações meteorológicas capturam a temperatura máxima, mínima, poluição e outros. As variáveis naturais tendem a ter um comportamento temporal cíclico, ou seja, elas apresentam sazonalidade que podem ser curtas como o dia e a noite ou longas como eclipses solares. Estas ciclicidades podem ser modeladas deterministicamente, entretanto, é complicado criar modelos do espaço e por isso utiliza-se de estimativas geoestatísticas. A modelagem geoestatística espaçotemporal requer um conjunto de dados estacionários de segunda ordem nos domínios do espaço e do tempo, assim deve-se considerar um procedimento para garantir isto. Matheron (1971) afirma que a presença de uma tendência não permite a estimativa direta do variograma. Deutsch (2002) comenta que o primeiro passo importante em qualquer modelagem geoestatística é estabelecer a variável correta para modelagem e garantir que esta propriedade pode ser modelada como uma variável estacionária no domínio estudado. $\mathrm{O}$ autor ainda discorre que se os dados apresentarem uma tendência sistemática, esta tendência pode ser removida antes da aplicação da geoestatística e devolvida posteriormente. Isto ocorre uma vez que a tendência deixa a variável não estacionária e a média fica dependente da localização. Chilès e Delfiner (1999) expõem que, na prática, assumir a estacionariedade temporal nunca é trivial e exige uma seleção meticulosa dos dados para garantir que eles se originem do mesmo processo físico subjacente.

A previsão espaçotemporal com deriva externa temporal local depende de duas considerações das séries temporais: (i) as tendências determinísticas podem ser extrapoladas para fazer previsões, portanto, pode-se justificar a projeção alegando que as tendências fundamentais geralmente mudam lentamente em comparação com o tempo de previsão (Cowpertwait e Metcalfe, 2009); (ii) o objetivo é estimar e definir os elementos determinísticos, tendência linear $(\operatorname{tr}(t))$ e sazonalidade $(\operatorname{sea}(t))$, esperando que o componente residual ou de ruído $\operatorname{res}(t)$ seja uma série temporal estacionária (Brockwell e Davis, 2010). Fazer uma 
previsão é inferir a distribuição probabilística do futuro a partir de uma população amostral de valores passados (Box et al. 2015) e do ponto de vista do modelo de regressão, é um valor esperado condicionado às tendências passadas que continuam nas próximas etapas (Cowpertwait e Metcalfe, 2009).

Vários estudos espaçotemporais consideram a tendência temporal como funções determinísticas e a removem para trabalhar com os dados residuais. O modelo de variograma dos resíduos é ajustado para prever a variabilidade dos resíduos e as funções determinísticas adicionam a tendência modelada e /ou ciclicidade. Hudson e Wackernagel (1994) aplicaram a elevação como deriva externa para ajudar na estimativa de um mapa de temperatura da Escócia. De Cesare et al. (1997) e De Cesare et al. (2001b) detectaram e removeram o efeito sazonal, inspecionando cada série temporal e estimando os resíduos para obter os valores no futuro e apenas no local monitorado. Sherman (2011) formulou um modelo geral de espaço-tempo com uma função puramente espacial, função puramente temporal e o processo de erro espaço-tempo intrinsecamente estacionário, assim, ele considera que esse modelo é suficientemente flexível para modelar uma grande variedade de conjuntos de dados espaçotemporais. Além disso, Cressie e Wikle (2011) decompõem o espaço-tempo considerando o caso em que a estrutura dinâmica é desconhecida e pode ser escrita em termos dos meios determinísticos e quatro efeitos aleatórios com média zero estatisticamente independentes: (1) variabilidade específica comum a todos os tempos; (2) variabilidade específica do tempo comum a todos os locais; (3) capturar a interação espaçotemporal; (4) variabilidade espaçotemporal da microescala. De forma semelhante, De Iaco e Posa (2012) comparam três formas de decomposição no espaço-tempo: (1) componente de tendência é constante no domínio espaço-tempo; (2) componente de tendência é descrito por um modelo determinístico no espaço e no tempo; (3) componente de tendência é descrito por um modelo estocástico no espaço e no tempo. Todas as interpolações foram estimadas para os locais amostrados a fim de comparar os seus resultados. De Iaco et al. (2015) utilizaram análises geoestatísticas dos resíduos obtidos da decomposição da componente de tendência em sub-regiões da área estudada. Menezes et al. (2016) e Monteiro et al. (2017) modelaram a tendência aplicando modelos lineares generalizados com uma componente aleatória e uma função de regressão linear com componentes de diferentes escalas para atingir resíduos estacionários. Outros autores, tais como Sølna e Switzer (1996), Adamowski e Bocci (2001) e Paci et al. (2013) utilizaram diferenciação para remover a tendência e sazonalidade para adquirir os resíduos. A análise de séries temporais para previsão regional foi aplicada por 
Cliff e Ord (1975) e Pfeifer e Deutsch (1980) que utilizam um processo de construção de modelos espaçotemporais com o ARIMA (STARIMA).

Outra ferramenta abordada por diversos autores consiste em utilizar uma informação auxiliar para modelar a tendência. Kilibarda et al. (2014) utilizaram a geometria da variável temperatura, ou seja, um modelo em função do dia do ano e localização (latitude). Kilibarda et al. (2015) consideraram valores medidos por imagens de satélites na função de regressão. Snepvangers et al. (2003) adicionaram uma informação dinâmica secundária (precipitação) para modelar a tendência da água no solo. Wang et al. (2017) estimaram a inclinação reta de tendência linear em cada pixel usando um modelo de elevação digital de terreno como dado secundário. 


\section{MÉTODO DE SIMULAÇÃO ESPAÇOTEMPORAL GEOESTATÍSTICA COM DERIVA EXTERNA TEMPORAL LOCAL}

\subsection{Proposta}

Muitas vezes, os procedimentos citados anteriormente são o suficiente para garantir a estacionariedade de segunda ordem da estimativa. Porém, eles podem não ser adequados para uma análise baseada em dados que têm variáveis espaçotemporais com grande oscilação espacial nos padrões temporais, ou seja, que a tendência e a ciclicidade temporal dependem da sua posição no espaço. Com a falta de métodos geoestatísticos que conseguem modelar a variabilidade temporal local, a simulação espaçotemporal geoestatística com deriva externa temporal local foi desenvolvida para medir a variabilidade espacial dos parâmetros de decomposição de séries temporais. Estes parâmetros são variáveis regionalizadas, ou seja, entre outras propriedades, eles têm posições no espaço definidas e podem ser interpolados por geoestatística a fim de gerar mapas das possibilidades futuras. Com os parâmetros mapeados, pode-se construir as tendências e sazonalidades das séries temporais para todos os pontos da região sendo estudada. Assim, construir, deterministicamente, os cenários prováveis da variável sendo estudada. Enquanto isso, os resíduos podem ser simulados por geoestatística espaçotemporal, uma vez que a remoção da tendência garante a estacionariedade de segunda ordem. Ou seja, a simulação espaçotemporal geoestatística com deriva temporal local decompõe as componentes de séries temporais em cada ponto estudado, mapeia estas componentes por geoestatística espacial e calcula para qualquer momento no tempo os seus valores. Enquanto isso, simulam-se os resíduos por geoestatística espaçotemporal para o mapeamento dos valores, incertezas e possíveis cenários da variável em estudo. O fluxograma deste procedimento pode ser visto na Figura 19. 


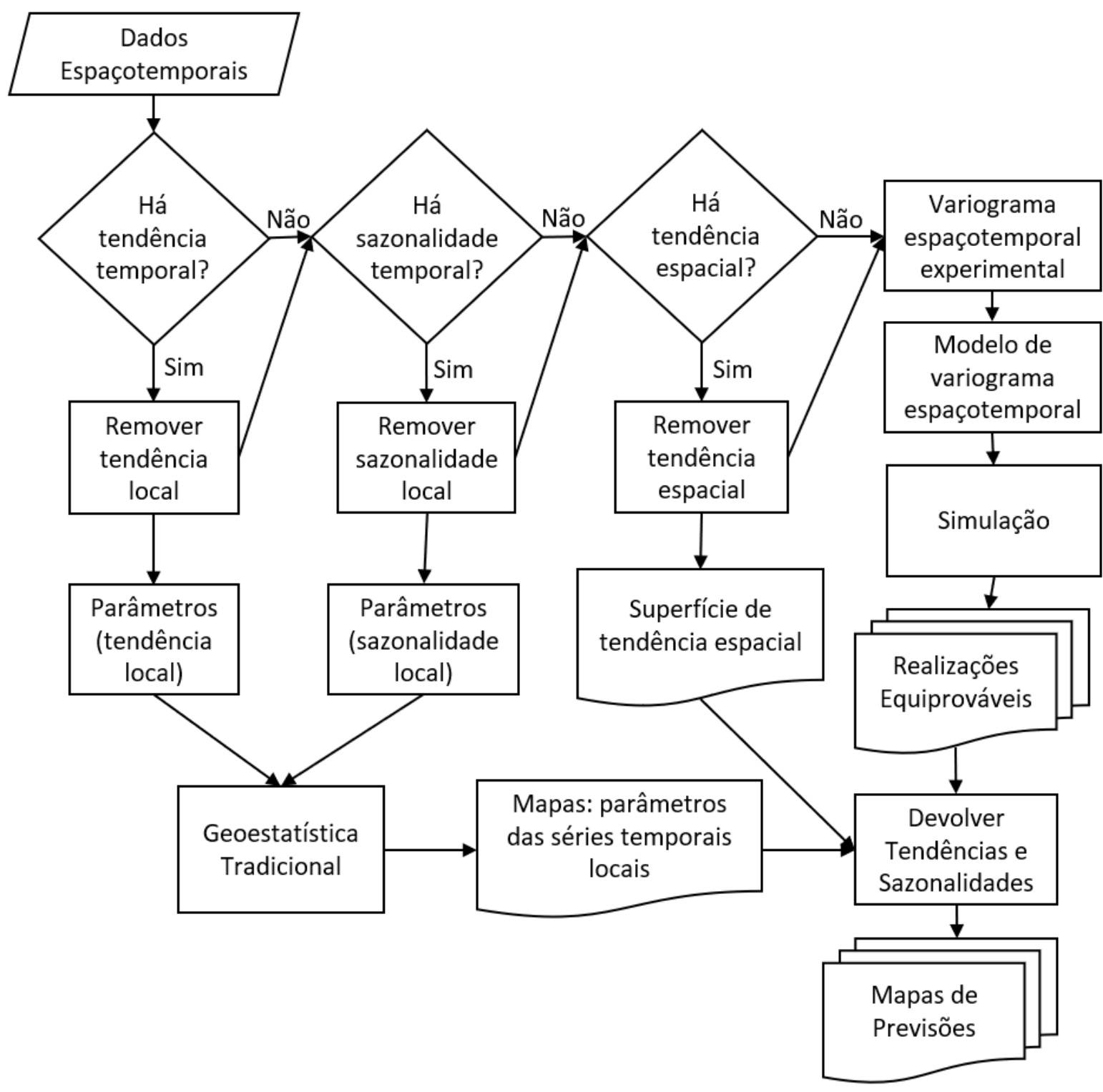

Figura 19 - Fluxograma mostrando os passos da simulação geoestatística com deriva temporal local

O método proposto transforma cada série temporal na sua forma mais simples com média zero (componente aleatória) somados a variabilidade espaçotemporal intrínseca ao fenômeno (não separável) e as oscilações de diversas frequências (i.e. El niño, La niña, Madden Julian, ciclos de Milankovitch e outros). Para isso, a decomposição das componentes das séries temporais é aplicada em cada ponto amostral. Desta maneira, a equação é: 


$$
Z(s, t)=U(s, t)+\beta(s, t)
$$

Onde $U(s, t)$ é o resíduo espaçotemporal estacionário e $\beta(s, t)$ é a série temporal (tendência e sazonalidade). Considerando que tanto a equação espaçotemporal quanto a série temporal $\beta(s, t)$ podem ser decompostas como demonstrado nas equações (Eq. 48) e (Eq. 49), pode-se substituir os componentes das análises de séries temporais e de maneira similar a tendência espacial (opcional) resultando com o processo espaçotemporal, conforme:

$$
Z(s, t)=U(s, t)+b_{1}(s)+b_{2}(s) t+R(s) \cos (\omega t+\phi(s))
$$

Onde $b_{1}(s)+b_{2}(s) t$ é a tendência temporal em cada ponto espacial s, $R(s) \cos (\omega t+$ $\phi(s))$ é a sazonalidade para cada ponto s e $U(s, t)$ é o resíduo espaçotemporal estacionário. Se for necessário, pode-se remover a tendência espacial também para garantir sua estacionariedade de segunda ordem no espaço. E o resíduo será uma mistura de efeitos aleatórios e variabilidades de pequena escala. Com a decomposição temporal e espacial (opcional), a estacionariedade de segunda ordem exigida pela geoestatística deve ser alcançada. Assim, os resíduos podem ser extrapolados para o futuro utilizando os métodos de simulação geoestatística (i.e., simulação por bandas rotativas).

Além disso, os parâmetros das séries temporais dependem de sua localização s no espaço, então estes pontos podem ser considerados como variáveis regionalizadas e mapeados por geoestatística espacial ou outro método de interpolação espacial (spline, inverso da distância ou outros). Os métodos de geoestatísticas devem ser priorizados devido a sua minimização da variância e possível estudo da anisotropia global. Uma vez que se obtém os mapas de todos os parâmetros das séries temporais (coeficiente angular $\left(b_{2}\right)$, intercepto $\left(b_{1}\right)$, amplitude $(\mathrm{R})$ e fase $(\phi))$, pode-se criar modelos determinísticos da tendência e sazonalidade para cada pixel da área de estudo.

Por último, é possível juntar os valores simulados para o período desejado com os valores determinísticos das séries temporais de cada ponto. Assim, devolve-se a tendência e sazonalidade ao resultado simulado. Este método permite simular mapas em qualquer local e instante, ou seja, passado, período amostrado ou futuro. É importante ressaltar que as séries 
temporais são criadas a partir de um período amostrado e deve-se considerar as limitações desta amostragem. Isso é, existe uma possível limitação na informação espacialmente (para mapear) e temporalmente (para séries temporais). A extrapolação temporal deve ser feita com cautela e considerar que a natureza que rege os dados no período amostrado não é alterada para os instantes simulados. Portanto, não é recomendado realizar pesquisas com períodos muito longos, pois possivelmente as forças naturais que causam o fenômeno podem ser alteradas.

A fim de validar o método proposto, ele é comparado com o estudo sem considerar deriva externa e com deriva externa global. Que são os métodos encontrados na literatura e foram utilizados com base para o desenvolvimento deste método, conforme já explicado.

\subsection{Publicações Científicas}

A pesquisa realizada no doutoramento resultou em três artigos publicados em periódicos internacionais:

i. Groundwater Level Prediction/Forecasting and Assessment of Uncertainty Using SGS and ARIMA Models: A Case Study in the Bauru Aquifer System (Brazil) (Takafuji et al., 2018)

ii. $\quad$ Spatio-temporal Kriging to Predict Water Table Depths from Monitoring Data in a Conservation Area at São Paulo State, Brazil (Manzione et al., 2019)

iii. Spatiotemporal forecast with local temporal drift applied to weather patterns in Patagonia (Takafuji et al., 2020)

Além disso, foram publicados dois trabalhos completos em anais de congresso:

i. Forecasting groundwater levels and assessing its uncertainty using SGS and ARIMA

- a case study in the Bauru aquifer system - Brazil (Takafuji et al., 2016)

ii. Geostatistics as a Tool to Map the Spatio-Temporal Evolution of Car Ownership in São Paulo Metropolitan Area (Takafuji et al., 2017)

e três resumos publicados em anais de congressos:

i. Modelling spatio-temporal variability of water table depths monitoring data (Manzione et al., 2016) 
ii. Spatio-temporal interpolation of water table depths in a conservation area at Bauru Aquifer System (SP/Brazil) (Manzione et al., 2017)

iii. Exploring the spatio-temporal nature of shallow groundwater monitoring data using geostatistical approach (Manzione et al., 2018) 


\section{ESTUDO DE CASO}

\subsection{Introdução do Estudo de Caso}

A modelagem numérica do clima tenta reproduzir a interação de diversas variáveis com o meio ambiente. Estes modelos são extremamente sensíveis às suas condições iniciais e qualquer variação pode levar a um cenário completamente diferente. Além disso, os modelos têm alta complexidade computacional e grande quantidade de dados (temperatura, pressão atmosférica, radiação solar, medidas de precipitação entre outras) o que faz o processamento durar longos períodos. Stull (2000) explica que, de maneira geral, as previsões meteorológicas são feitas através do cálculo de equações de movimento da atmosfera, equações parciais diferenciais não lineares de dinâmica, conservação de umidade, termodinâmica e continuidade de massa. O objetivo destas equações é acompanhar a infinidade de interações não-lineares complexas entre ventos, temperatura e umidade em milhares de locais e altitudes ao redor do mundo. Assim, a modelagem numérica tenta adicionar uma melhor compreensão do modus operandi do sistema climático da Terra (Lau, 2006). Porém, alguns processos físicos não são compreendidos o suficiente para guiar suas leis físicas exatas ou os processos físicos exatos são tão complicados (computacionalmente) que, nessas circunstâncias, uma parametrização mais simples pode fornecer um resultado aceitável (Stull, 2000). Henderson-Sellers e McGuffie (1997) mostram que os modelos climáticos podem ser considerados como uma simplificação do mundo real a partir de séries de equações que expressam leis físicas, químicas, biológicas e sociais. Além disso, quanto mais complexa a representação, mais difícil é utilizar o modelo e o resultado será apenas uma aproximação.

Em contraste com a modelagem climática, a geração de mapas de previsão de variáveis climáticas, como temperatura, pode ser feita com análises baseadas apenas nos próprios dados utilizando métodos de séries temporais para previsão e métodos de geoestatística espaçotemporais para interpolação. Isto é, os modelos geoestatísticos dependem apenas da autocorrelação espaçotemporal da variável em um passo estabelecido no espaço e um passo no tempo. Se a variável apresenta continuidade espaçotemporal, geralmente, ela pode ser modelada e estimada em qualquer ponto definido do espaço e do tempo. O estudo de um estimador de mapas futuros baseado apenas nos dados tem diversas vantagens, mas também desvantagens. A principal vantagem é que os modelos são processados mais rapidamente, são mais simples e diretos que os encontrados nas análises numéricas climatológicas e a principal desvantagem é os modelos não considerarem todos os possíveis parâmetros que podem afetar 
o clima na região. Na prática, a modelagem climática tenta reconstruir toda a evolução dos parâmetros estudados e como eles interagem entre si. Enquanto a geoestatística se baseia nas estatísticas espaçotemporais de uma variável para fazer uma previsão em um ponto (espaçotemporal) em que não se conhece o valor.

O estudo de análises de séries temporais pode reconhecer padrões e prever variáveis climáticas com uma determinada localização no espaço. Os métodos de análises de séries temporais utilizam funções matemáticas para capturar a essência da sua variação, métodos tradicionais utilizam modelos como autorregressão, média móvel, diferenciação e análise harmônica.

Espera-se que este método proposto seja especialmente robusto para áreas em que os parâmetros das séries temporais variam de acordo com sua posição no espaço. Ou seja, uma área grande com alta volatilidade das tendências temporais e de preferência com pouca informação. Este é o caso da Patagônia, onde existem poucas estações meteorológicas que fornecem informações climáticas para uma das primeiras regiões habitadas que podem ser afetadas pelo aquecimento global. O clima é influenciado pela Antártica, correntes marítimas de dois oceanos, cordilheiras e pradarias, o que faz que ele seja de árido a frio úmido. Assim, esta região é complexa para estudo climático e será essencial para avaliar a robustez do método proposto. O estudo compara três abordagens da geoestatística espaçotemporal: (i) análise geoestatística sem considerar deriva externa; (ii) análise com deriva externa global, ou seja, considera um modelo médio da variação temporal da temperatura e; (iii) análise com deriva externa temporal local, o qual utiliza um modelo de variação temporal da temperatura para cada estação meteorológica.

\section{2 Área de Estudo}

O estudo de caso foi realizado na região da Patagônia a fim de verificar se a hipótese de que o método proposto, simulação espaçotemporal com deriva externa temporal local, é robusto e adequado para mapear variáveis que apresentam variações cíclicas e tendências no tempo. $\mathrm{O}$ local foi escolhido devido à falta de informação, pois em uma área de mais de $10^{6} \mathrm{~km}^{2}$ apresentando apenas 18 estações meteorológicas cujas localizações podem ser observadas na Figura 20. Sendo assim, este estudo é considerado um caso extremo, pois é avaliada a qualidade do resultado de uma simulação baseada apenas nos próprios dados em uma região com pouca 
informação e alta variabilidade climática e extensão territorial. Além disso, a figura ainda destaca duas estações meteorológicas (vermelho) que serão utilizadas como referência para os exemplos das decomposições, elas foram selecionadas para mostrar como duas séries temporais se comportam no espaço. Comparando todas as estações, a estação A está em uma região central, tanto na sua localização continental, quanto na distribuição de valores de temperatura. Enquanto a estação B está no extremo sul da América do Sul e apesar de estar perto do mar, está numa porção continental e seus valores de temperatura são mais extremos. De modo geral, as estações meteorológicas estão bem distribuídas, ou seja, não existe um agrupamento preferencial, mas é possível observar que a região da Cordilheira dos Andes tem pouca informação. O shapefile da Patagônia usado foi fornecido pelo site Global Administrative Areas (2018). A climatologia ou condições meteorológicas médias de longo prazo dependem da relação entre alguns elementos como pressão atmosférica, nuvens, chuva, umidade relativa, vento (velocidade e direção) e outros (Eidt, 1969). Garcia (1994) comenta a climatologia da América do Sul e mostra que os padrões climático presentes no continente sul-americano apresentam ciclicidade bem estabelecida com quatro estações, principalmente a sul do trópico de Capricórnio. Além disso, as correntes marítimas circumpolares antárticas governam o clima no extremo austral do continente.

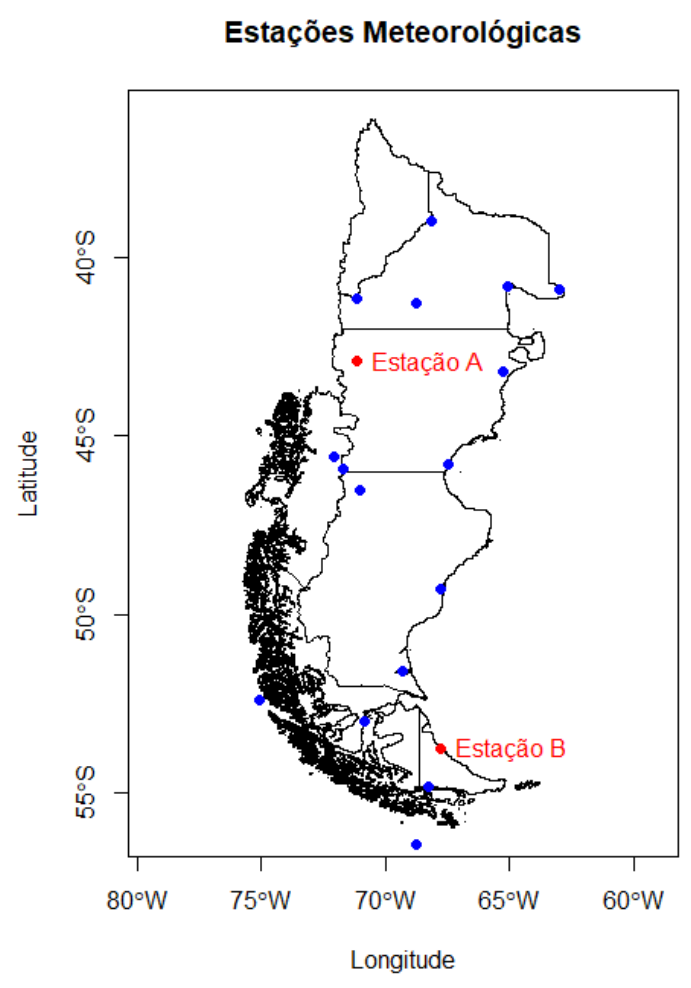

Figura 20 - Mapa da Patagônia e a localização das estações meteorológicas (azul) e vermelho estações que serão utilizadas de exemplo 


\subsection{Preparação dos Dados}

O banco de dados utilizado neste estudo foi fornecido pela plataforma Climate Data Online (CDO) da (National Climatic Data Center - NCDC) que faz parte da administração oceânica e atmosférica nacional (National Oceanic and Atmospheric Administrator - NOAA) o banco de dados é o "Resumo Global do Dia" (Glabol summary of the day - GSOD, 2018)

Este banco contém informações diárias e elas foram recalculadas como médias mensais para suavizar as oscilações de alta frequência (alta variabilidade diária) e melhorar a qualidade da informação, pois em muitos dias não foram coletadas informações. Sendo assim, considerouse necessário ter informação em pelo menos 10 dias no mês para o cálculo da média mensal. Além disso, as informações de algumas estações meteorológicas foram descartadas devido à baixa quantidade de dados mensais. As estações foram selecionadas seguindo as premissas: (i) o conjunto de dados na estação deve possuir pelo menos $25 \%$ dos meses medidos; (ii) deve haver pelo menos 24 meses de medidas contínuas e; (iii) ter dados recentes (preferencialmente). Sendo assim, se alguma premissa não for atingida, as informações da estação meteorológica são descartadas. Ao final da seleção, restaram apenas 18 estações meteorológicas na região da Patagônia.

O conjunto de dados (GSOD) das estações meteorológicas na região da Patagônia inicia o monitoramento com periodicidade adequada em 1973 e continua até o presente momento. Para realizar um estudo de previsão de tempo que seja razoavelmente longo ( 1 ano), o conjunto de dados foi dividido em período de calibração de 15 anos (de janeiro de 2002 a dezembro de 2016) e de controle (de janeiro a dezembro de 2017). Os dados do período de calibração são utilizados para os ajustes dos modelos (séries temporais e geoestatística). Enquanto os dados do período de controle são apenas para comparação e avaliação dos resultados, ou seja, são considerados como dados "futuros" que validam a qualidade dos métodos.

Além disso, a "temperatura da superfície da terra" ou Land Surface Temperature - LST (Wan et al., 2015) foi considerada como valores verdadeiros para avaliar os resultados espacialmente e calcular os erros em cada ponto estimado. O LST é o produto de uma imagem de satélite calculada para estimar a temperatura da superfície com uma resolução de $0,05^{\circ}$. Estas imagens foram adquiridas pela plataforma EarthData da NASA. 


\subsection{Decomposição Temporal de Modelos Determinísticos}

A análise de deriva externa temporal utiliza de modelos que são ajustados aos dados para definição das tendências e periodicidades temporais. Ou seja, é feito um ajuste determinístico que pode ser utilizado para remover a tendência temporal dos dados. Então, os resíduos devem ser estacionários e podem ser utilizados para estudos geoestatísticos.

Assim, os dados de todas as estações meteorológicas do período de calibração foram decompostos com a seção temporal da (Eq. 55) através de um ajuste por mínimos quadrados da equação que considera apenas a tendência geral e a sazonalidade anual (frequência de 12 meses). Com isso foi possível computar os valores dos resíduos $\mathrm{U}(\mathrm{s}, \mathrm{t}$ ) para os métodos testados.

O primeiro método de decomposição utilizado neste estudo é global e a segunda abordagem é a deriva externa temporal local que consiste em considerar um modelo individual de série temporal ajustado para cada estação meteorológica.

Os resultados dos ajustes dos modelos e os valores dos resíduos podem ser observados na Figura 21, onde fica claro a diferença entre os modelos ajustados para a estação A e para a estação B (a localização destas estações é destacada na Figura 20) para a decomposição Global. A estação B teve um ajuste pobre de sua série temporal, pois este método fez apenas o modelo médio de séries temporais e existem diferenças regionais nos padrões das séries temporais. Por outro lado, a decomposição local ajusta a tendência e a sazonalidade em cada estação, o que significa que cada ajuste depende apenas de sua própria série temporal local.

De acordo com a (Eq. 55), são necessários cinco parâmetros para modelar a equação determinística do tempo, estes parâmetros são: (i) Frequência (constante em doze meses); (ii) Amplitude (R); (iii) Intercepto $\left(b_{1}\right)$; (iv) Coeficiente angular $\left(b_{2}\right)$ e; (v) Fase $(\phi)$. Além disso, os fatores ii a $\mathrm{v}$ calculados pelo método de deriva externa temporal local podem ser considerados como variáveis regionalizadas, pois possuem localização específica no espaço. 
Estação A
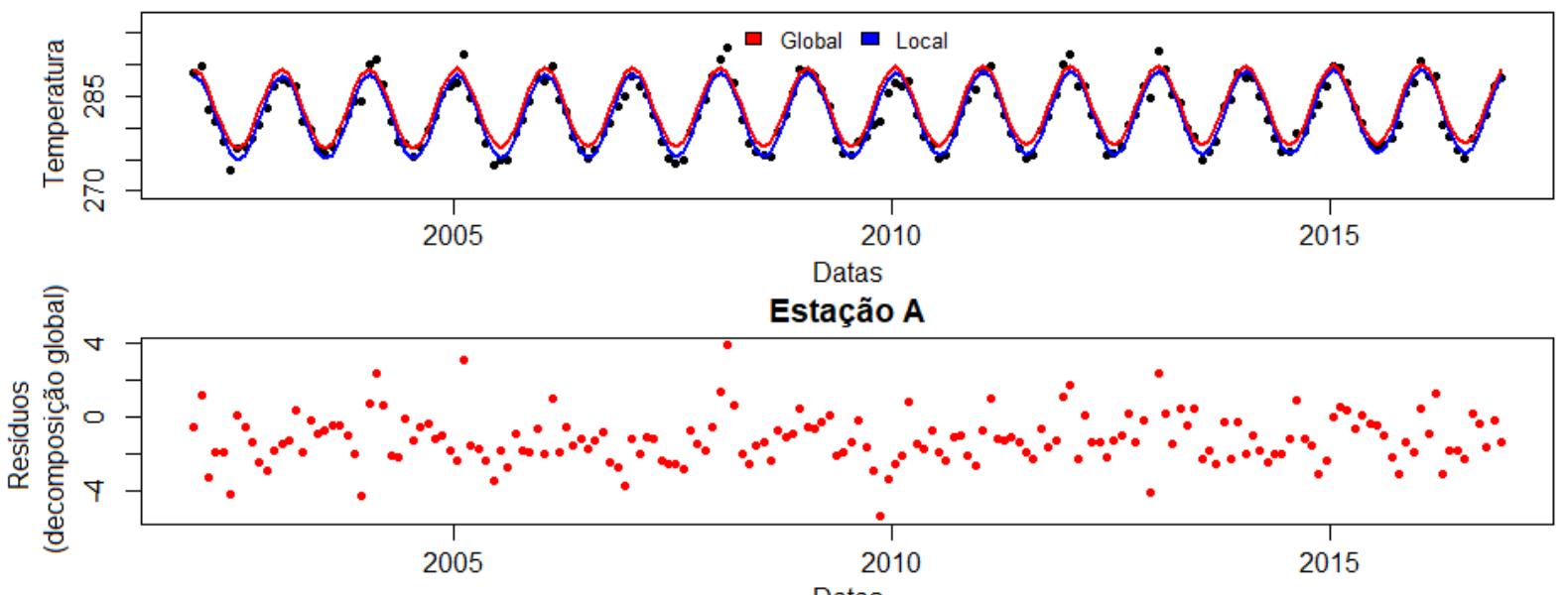

Datas

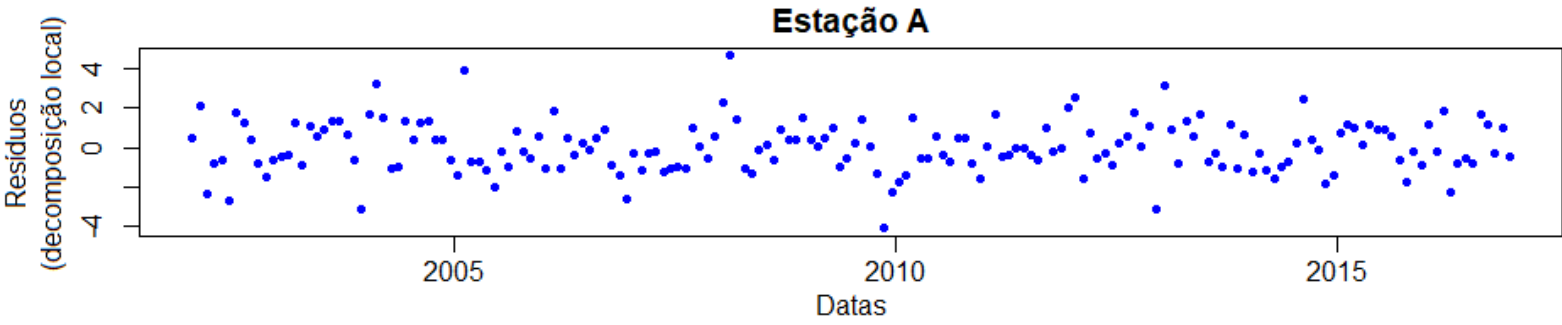

Estação B

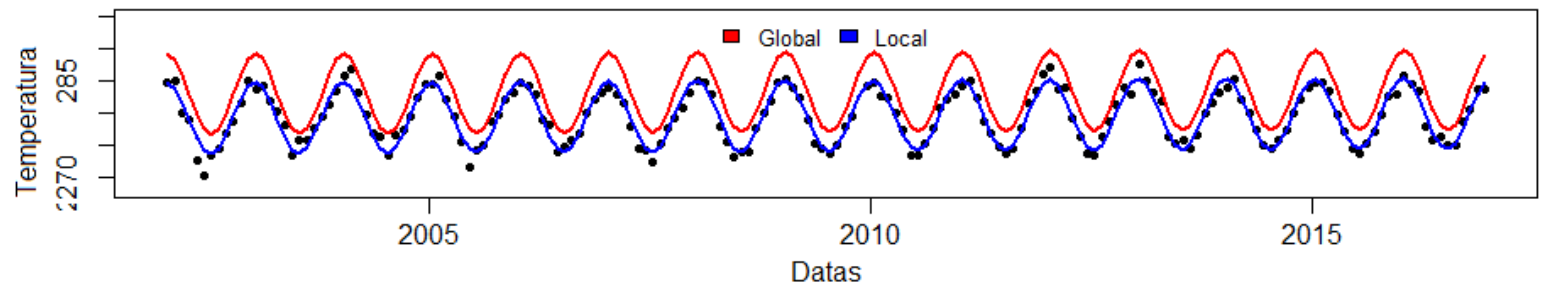

Estação B

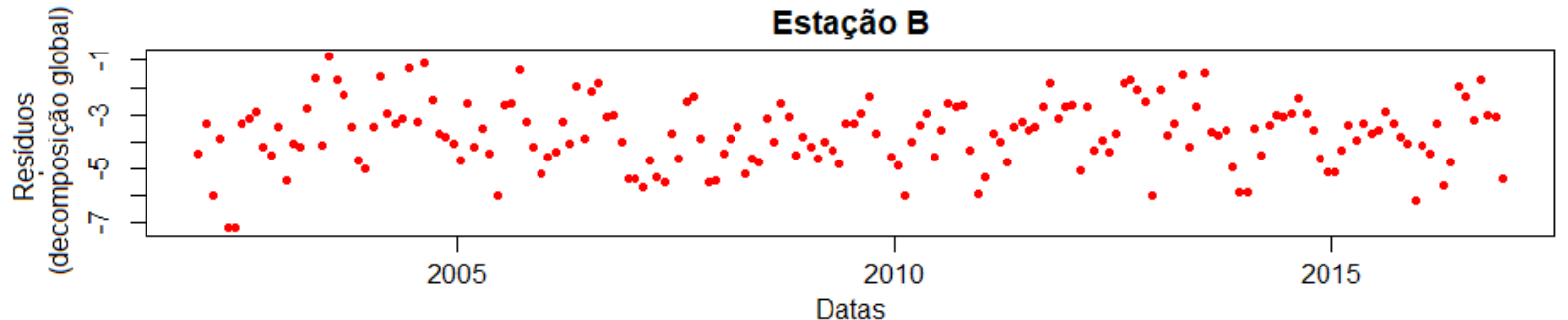

Estação B

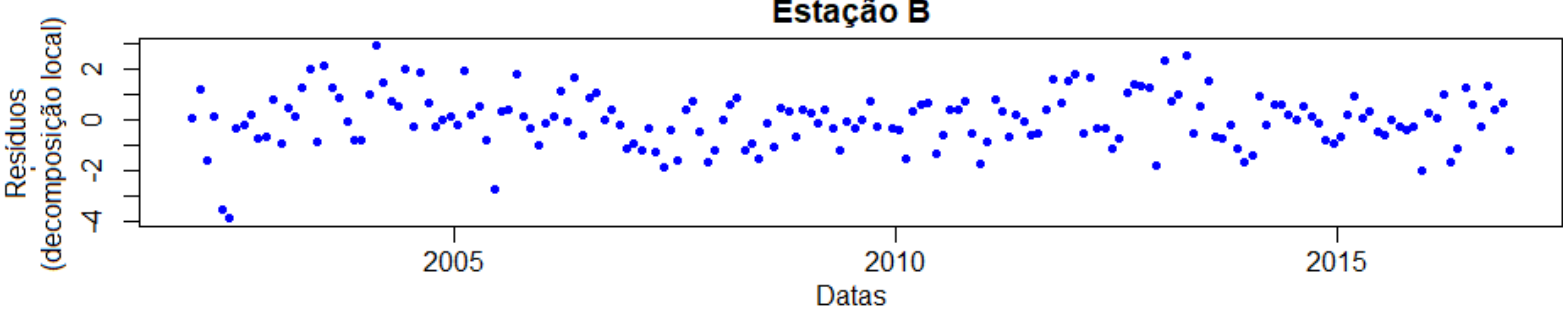

Figura 21 - Séries temporais (estações A e B) com a linha do ajuste seguido dos resultados decompostos em suas respectivas cores (vermelho para decomposição global e azul para local) 


\subsection{Análise: Estatística Descritiva}

A análise estatística foi feita a fim de estudar a distribuição da variável temperatura para as estações meteorológicas, tanto no período de calibração quanto no período de controle, além dos valores obtidos pelo produto LST da imagem de satélite. As comparações das estatísticas descritivas (dados totais, ou seja, sem discretização mensal) são apresentadas na Tabela 1 e de maneira gráfica, na Figura 22 o histograma e o boxplot dos dados de temperatura no período de calibração, de controle e LST. É possível observar que o conjunto de dados obtido pela imagem de satélite apresenta uma distribuição mais ampla, ou seja, os valores máximos e mínimos são maiores e menores, respectivamente, isto pode ser explicado devido aos dados de satélite apresentarem maior cobertura territorial, conseguindo capturar valores extremos e onde não existem estações meteorológicas. Além disso, os dados do período de controle e de calibração parecem ter distribuição semelhante com média, $\mathrm{P}(25)$ e $\mathrm{P}(75)$ próximos.

Tabela 1 - Estatística dos dados de temperatura em Kelvin das estações (calibração e controle e imagens de satélite (LST)

\begin{tabular}{cccccccc}
\hline & Min. & $\mathrm{P}(25)$ & $\mathrm{P}(50)$ & Média & $\mathrm{P}(75)$ & Máx. & $\begin{array}{c}\text { Desvio } \\
\text { Padrão }\end{array}$ \\
\hline Estações (Calibração) & 269,90 & 278,90 & 282,30 & 283,30 & 287,10 & 299,50 & 5,82 \\
Estações (Controle) & 273,20 & 279,00 & 282,80 & 283,60 & 287,20 & 299,00 & 5,86 \\
LST & 256,30 & 274,10 & 279,10 & 280,90 & 286,80 & 305,90 & 9,01 \\
\hline
\end{tabular}

Histograma

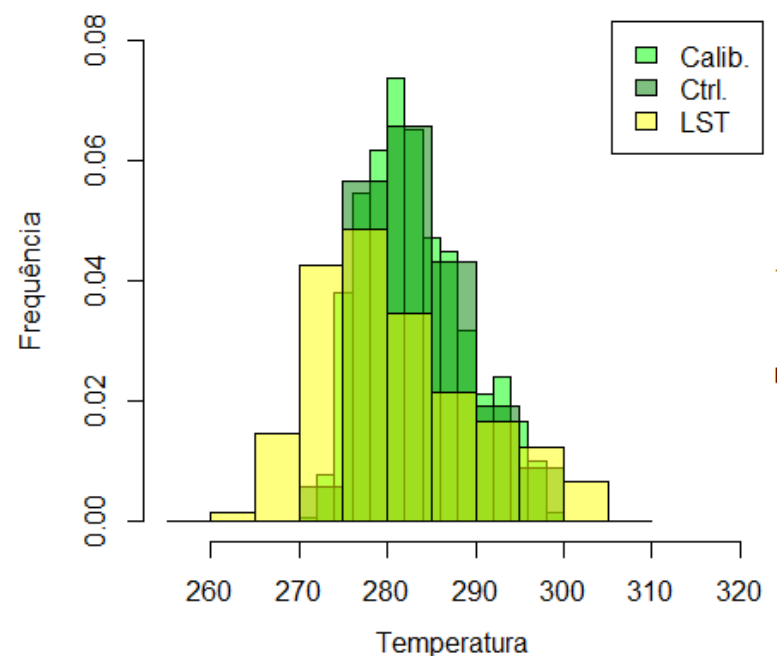

Boxplot

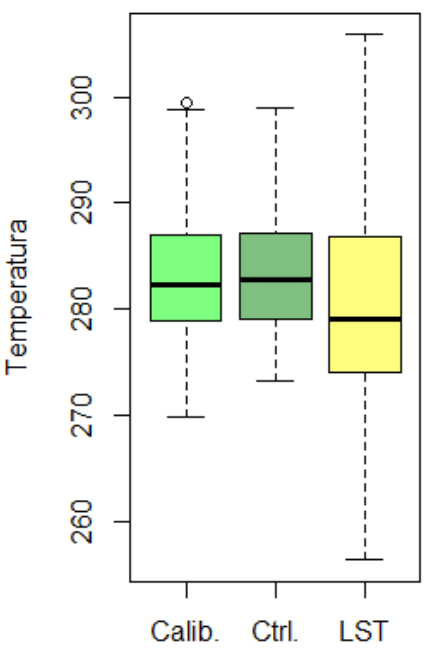

Figura 22 - Histograma e boxplot dos dados de temperatura (em Kelvin) das estações (calibração e controle) e imagens de satélite (LST) 
Após as decomposições global e local, as estatísticas descritivas (Tabela 2) mostram que ambos os resíduos têm média próxima a zero, porém sua comparação com o $\mathrm{P}(50)$ mostra que a decomposição global é assimétrica. Além disso, os valores extremos mostram que a decomposição local é mais precisa, pois os valores variam menos em torno da média. Isto fica claro na Figura 23, onde é possível ver que o boxplot e o histograma do método de decomposição local mostra baixa distribuição ao redor do zero, quando comparado com os gráficos do método global.

Tabela 2 - Estatística descritiva dos dados de temperatura decompostos (global e local) das estações meteorológicas.

\begin{tabular}{cccccccc}
\hline & Min. & $\mathrm{P}(25)$ & $\mathrm{P}(50)$ & Média & $\mathrm{P}(75)$ & Máx. & $\begin{array}{c}\text { Desvio } \\
\text { Padrão }\end{array}$ \\
\hline Global & $-10,02$ & $-2,72$ & $-0,74$ & $-0,02$ & 3,15 & 10,21 & 3,76 \\
Local & $-5,58$ & $-0,75$ & 0,02 & 0,00 & 0,74 & 6,39 & 1,20 \\
\hline
\end{tabular}

Histograma

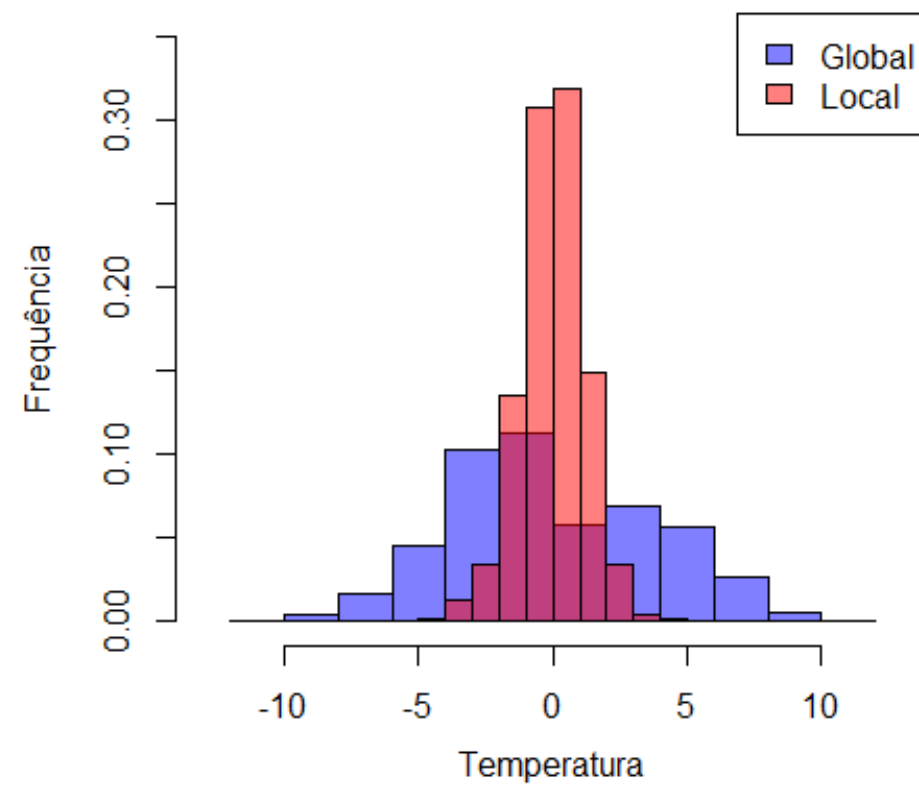

Boxplot

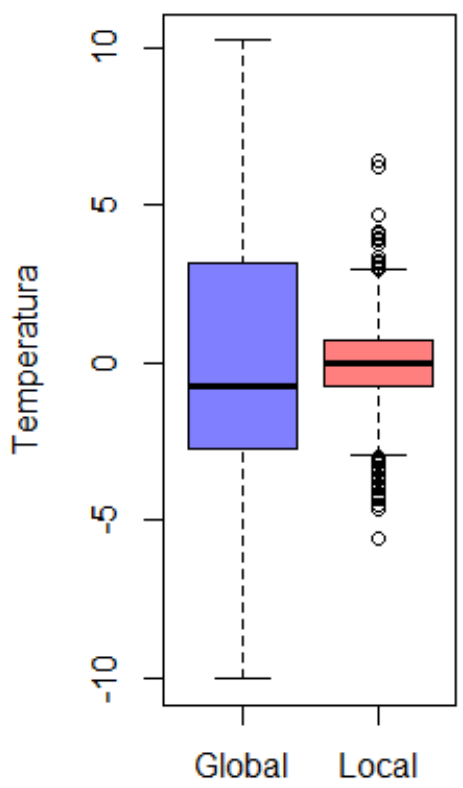

Figura 23 - Histograma e boxplot dos dados de temperatura decompostos globalmente e localmente

A fim de avaliar a acurácia dos modelos de séries temporais ajustados, a raiz do erro quadrático médio (Root Mean Square Error - RMSE) foi utilizado para medir o erro de cada série temporal (estação meteorológica). Hyndman e Koehler (2006) explicam que o RMSE é 
muito utilizado devido a sua relevância teórica na modelagem estatística com a vantagem de estar na mesma escala dos dados. Os autores supracitados expressam a equação como

$$
R M S E=\sqrt{E\left[e_{t}^{2}\right]}
$$

onde $e_{t}^{2}$ é o erro (valor amostrado menos o valor do modelo de serie temporal) e é calculado para cada t.

As estatísticas dos erros calculados para cada série temporal (estação meteorológica) são apresentados na Figura 24, onde é possível ver que o erro da decomposição local é muito mais baixo (RMSE $=1,19)$ que o erro da decomposição global (RMSE $=3,47)$. Isto mostra que os modelos ajustados pela decomposição temporal local são mais adequados para estudos em que as séries temporais têm padrões diferentes e que dependem da sua posição no espaço.

Histograma do RMSE

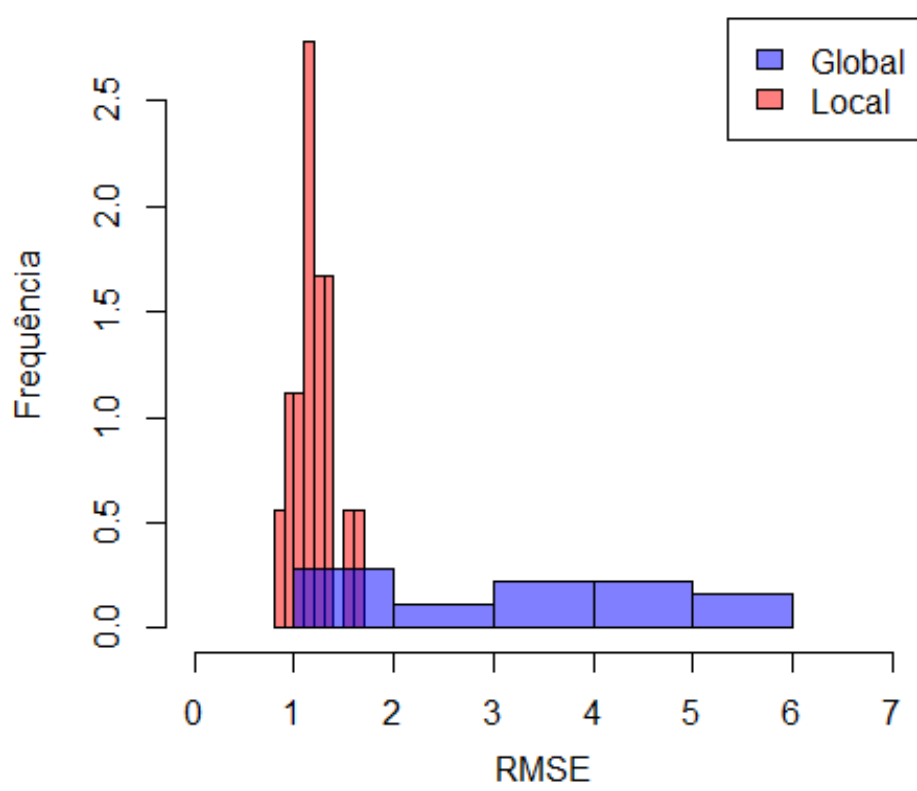

Boxplot

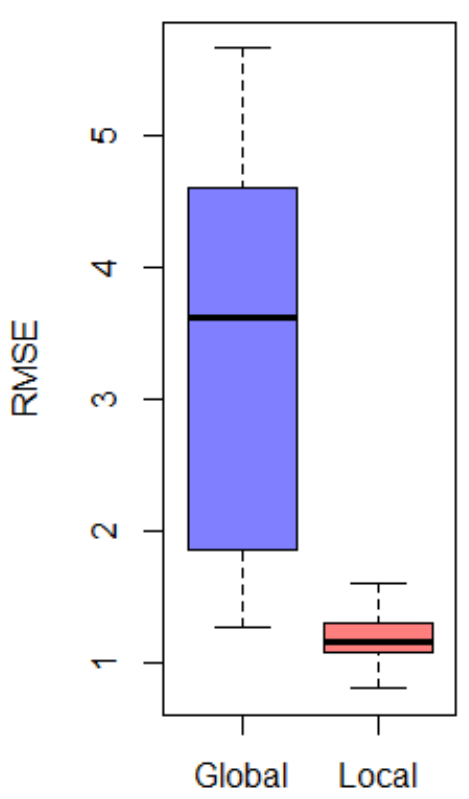

Figura 24 - Histograma e boxplot da raiz da média do quadrado do erro (RMSE) dos dados decompostos globalmente e localmente 


\subsection{Análise: Séries Temporais}

Outra maneira de comparar e validar os resultados é aplicar as técnicas de séries temporais como estimativas por modelos autoregressivos integrados com médias móveis (ARIMA). Estes modelos tentam capturar a essência da oscilação temporal misturando técnicas de diferenciação, médias móveis e autocorrelação. Estes modelos são ajustados para os dados amostrais e extrapolados para fazer previsões futuras. A Figura 25 mostra a previsão das séries temporais pelos modelos de ARIMA, a comparação é feita entre a série temporal original (sem decomposição) e com decomposições global e local. Ambas as estações meteorológicas (A e B) foram bem modeladas no espaço original e, consequentemente, os resultados da previsão por séries temporais parece adequada. Além disso, os resultados encontrados nas séries temporais dos dados decompostos mostram que os valores futuros ficam estáticos no valor médio para a estação A, ou seja, os modelos de ARIMA não conseguiram capturar nenhuma tendência ou sazonalidade. Isto indica que os modelos determinísticos removeram estas componentes. Enquanto para a estação B, os modelos de ARIMA capturaram pequenas oscilações, principalmente no modelo da decomposição global. A incerteza dos dados decompostos cobre quase todo o espaço de possibilidades, pois os resíduos são compostos de oscilações não modeladas e componentes aleatórias.

Mais uma maneira de comparar os resultados com análises de séries temporais é estudar a função autocorrelação (ACF) das séries temporais. Isto é, a correlação da variável com ela mesma em outro momento no tempo. A Figura 26 mostra a ACF das estações meteorológicas A e B com os dados brutos e com decomposições global e local. É clara a correlação temporal que os dados brutos apresentam, ela também é conhecida como memória temporal, pois ela indica o quão correlacionados são os dados em diferentes períodos. Mais uma vez é possível observar que a decomposição local deixou a ACF com patamares menores tanto para a estação A quanto para a estação B. Este é mais um indicador que a decomposição local capturou melhor as oscilações temporais das estações meteorológicas. 

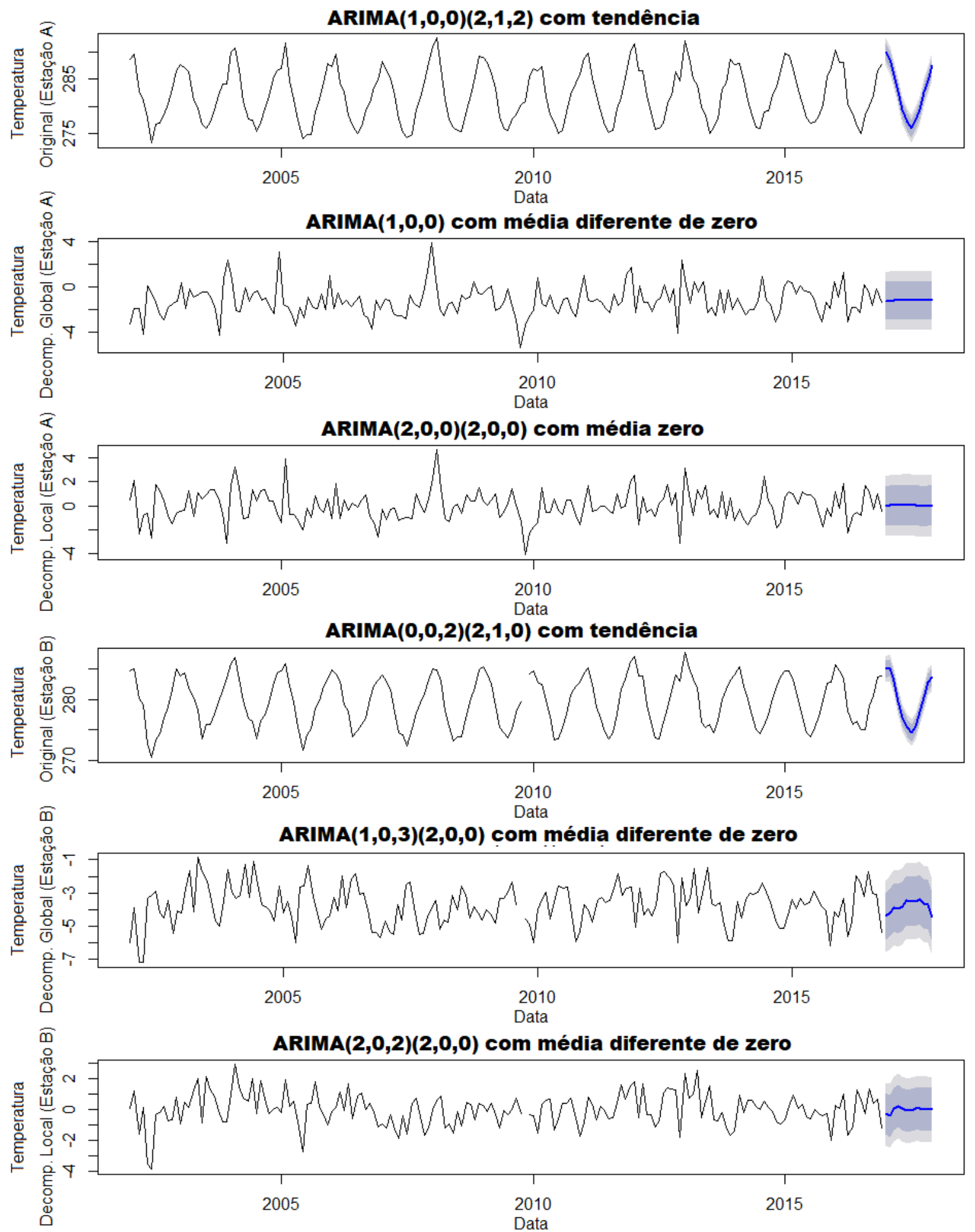

Figura 25 - Séries temporais (estações A e B) com a previsão por ARIMA dos dados brutos e decompostos (globalmente e localmente). A linha azul é o valor esperado, o campo cinza escuro é com incerteza de $80 \%$ e em cinza claro $95 \%$ 
Bruto (Estação A)

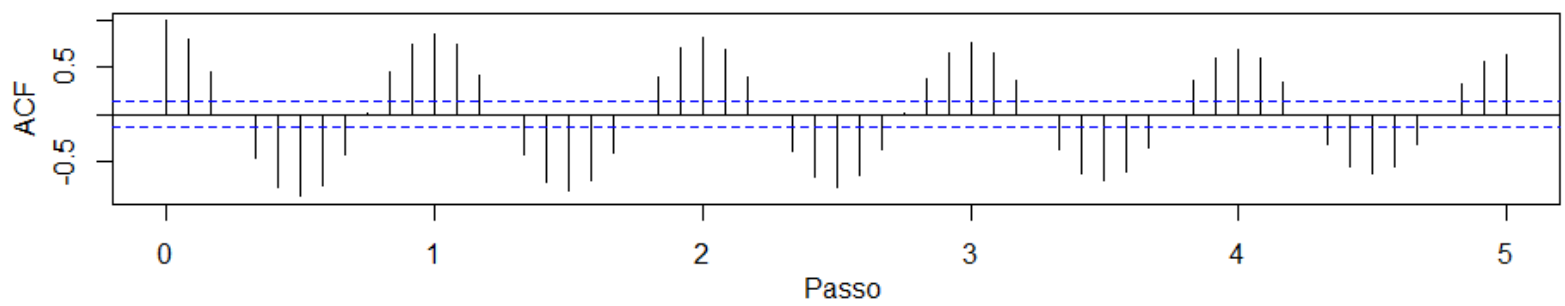

Decomp. Global (Estação A)

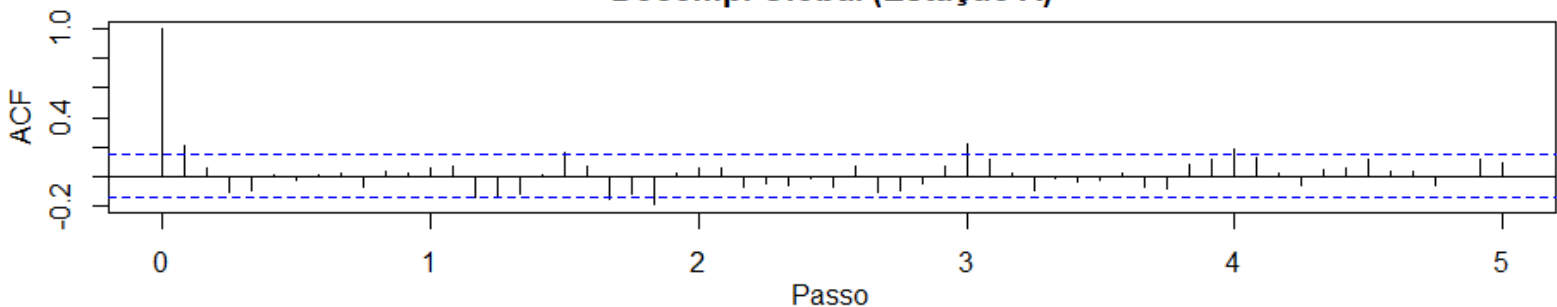

Decomp. Local (Estação A)

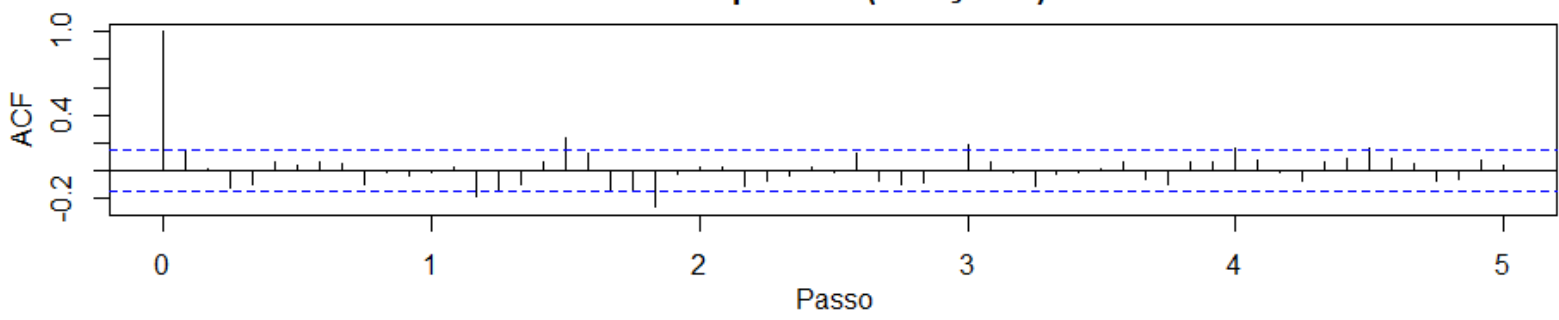

Bruto (Estação B)

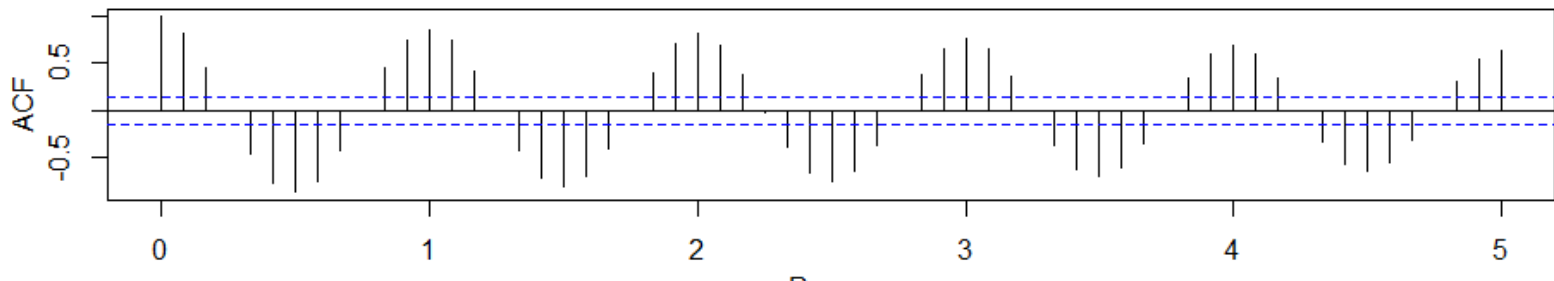

Passo

Decomp. Global (Estação B)
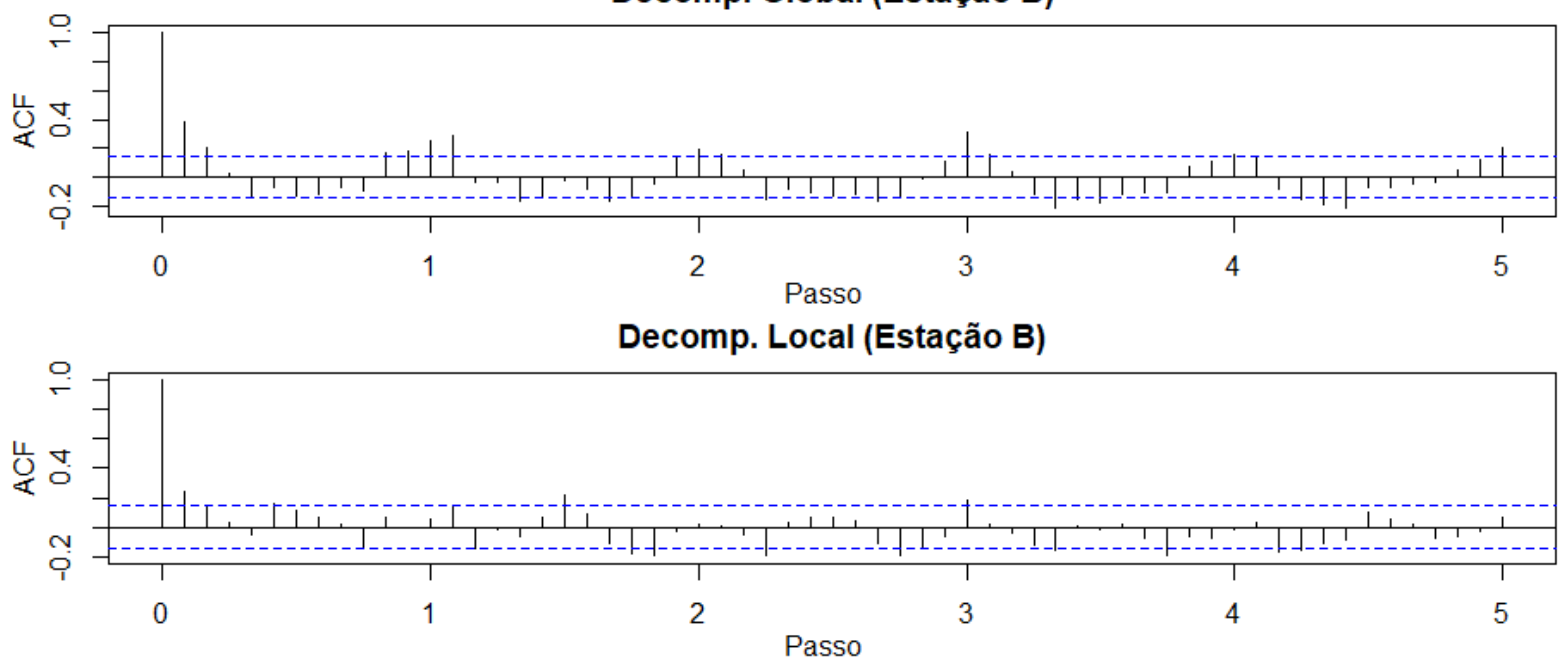

Figura 26 - Função autocorrelação (ACF) das estações A e B dos dados brutos e decompostos (globalmente e localmente). A linha azul é o intervalo de confiança de $95 \%$ 


\subsection{Mapeamento dos Parâmetros das Séries Temporais}

Os quatro parâmetros obtidos pela decomposição local (amplitude, intercepto, coeficiente angular e fase) podem ser considerados como variáveis regionalizadas, pois eles apresentam localização no espaço, suporte amostral, continuidade espacial e possível anisotropia. Assim, a melhor alternativa para estimar espacialmente esses parâmetros é a aplicação das técnicas de krigagem, porém se não houver variograma estruturado na região pode-se utilizar técnicas de interpolações como inverso da distância, vizinho mais próximo, funções de bases radiais, spline e outros.

Neste estudo, apenas a amplitude e a fase possuem variogramas estruturados e modeláveis (Figura 27), porém a anisotropia não pode ser reconhecida, provavelmente devido à pequena quantidade de amostras. A tendência espacial foi verificada e a amplitude mostra um variograma estruturado quando o polinômio espacial de primeira ordem foi ajustado e removido. Os outros parâmetros, coeficiente angular e intercepto não apresentam variogramas estruturados (Figura 28) ou seja, apresentam efeito pepita puro. A região possui apenas 18 estações meteorológicas e provavelmente mais estações enriqueceriam esses modelos. $\mathrm{O}$ variograma da amplitude foi modelado com o modelo esférico com efeito pepita de 0,3 , variância espacial em 0,73 e amplitude igual a 4. Além disso, o variograma da fase foi modelado com o modelo esférico, sem efeito pepita, patamar em 0,01 e amplitude igual a 6,5.

Para fins de mapeamento, o tamanho da célula escolhido é de $0,5^{\circ}$ em latitude e longitude. E a krigagem ordinária foi utilizada com uma vizinhança de busca global. Optou-se para as variáveis que ficaram sem modelos de variograma pela interpolação pelo inverso do quadrado da distância. As figuras que apresentam a distribuição espacial da variável regionalizada e o mapa estimado ou interpolado da amplitude, coeficiente angular, fase e intercepto são, respectivamente a Figura 29, Figura 30, Figura 31, Figura 32. 


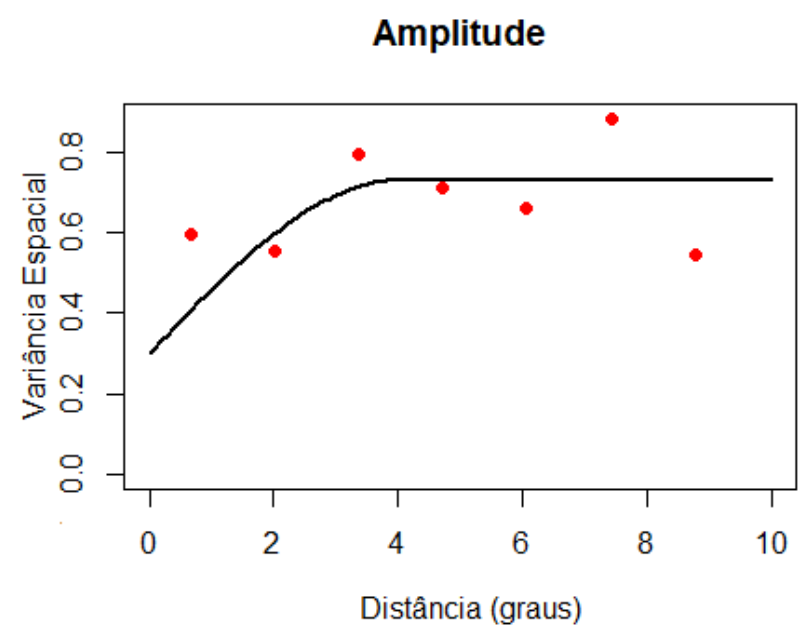

Figura 27 - Variograma experimental (pontos vermelhos) e modelo (linha preta) das variáveis regionalizadas amplitude e fase

Coe. Ang.

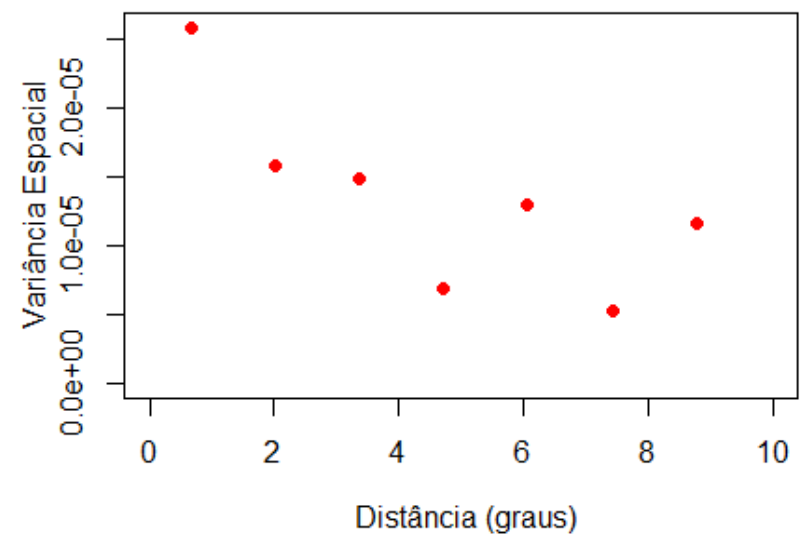

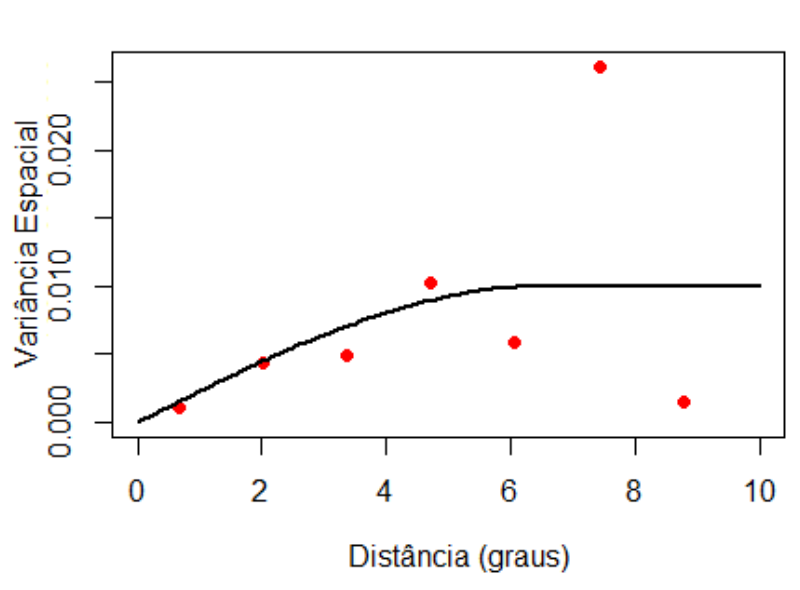

Fase 

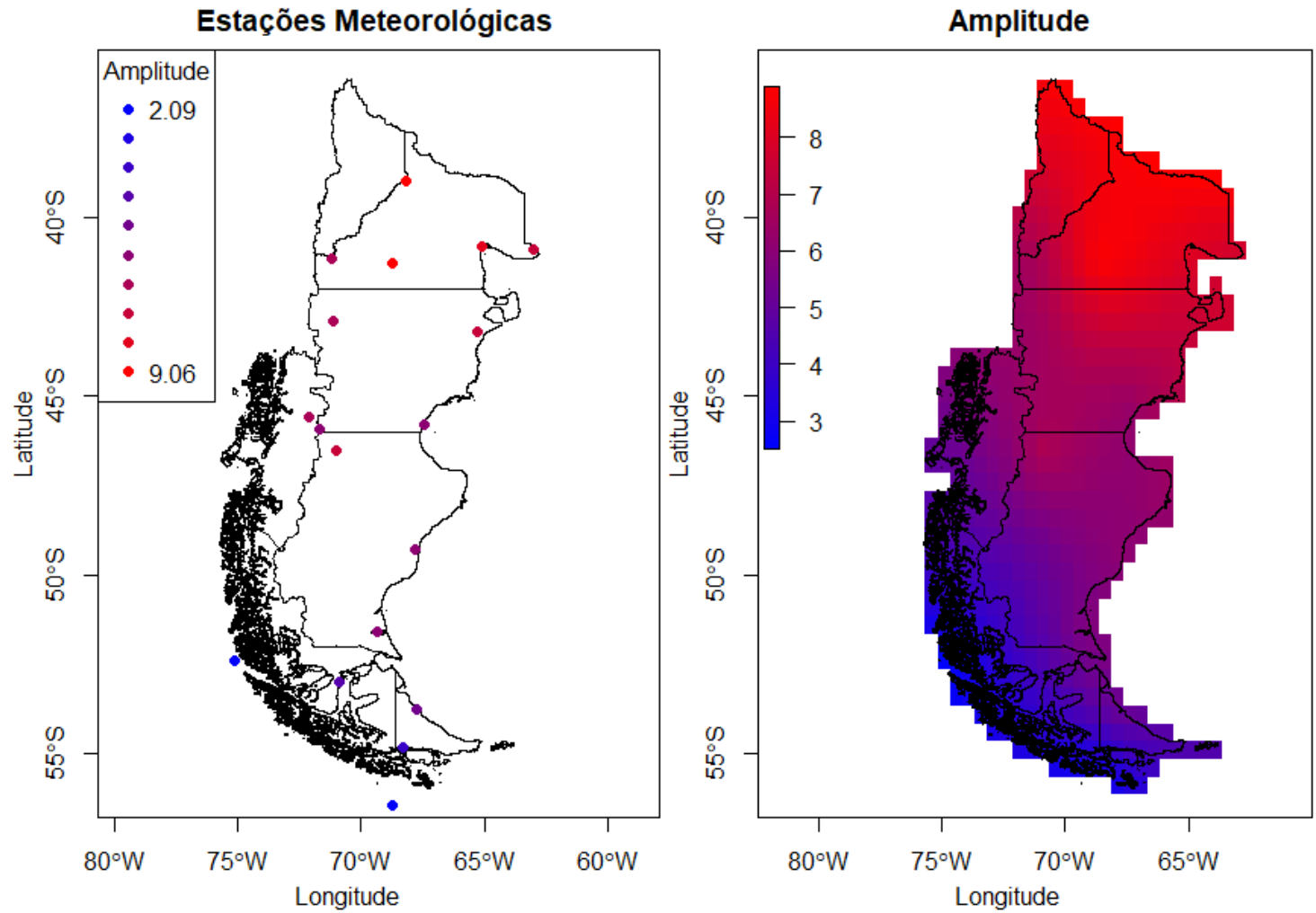

Figura 29 - Distribuição espacial da variável regionalizada amplitude e seu mapa krigado
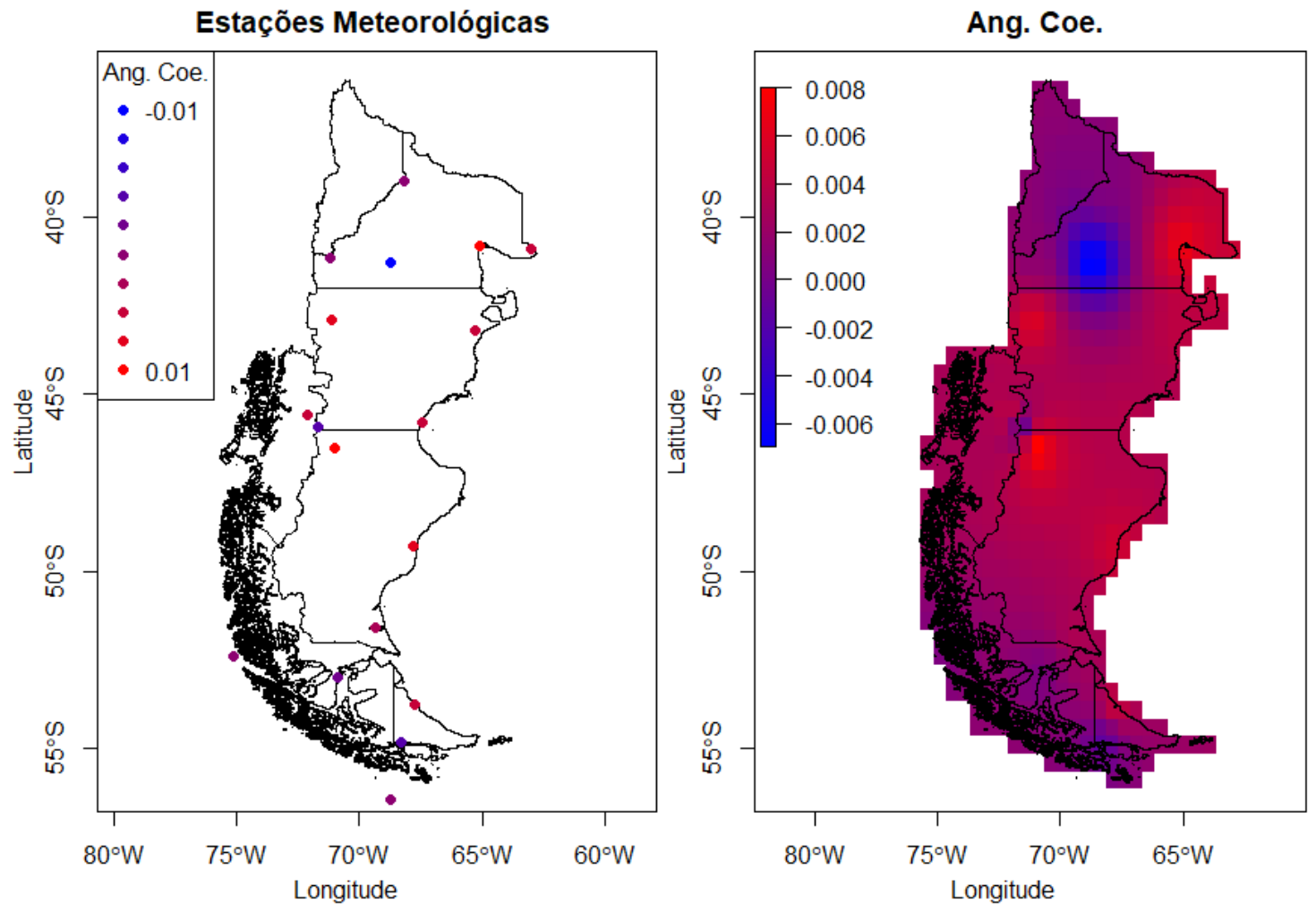

Figura 30 - Distribuição espacial da variável regionalizada coeficiente angular e seu mapa interpolado 

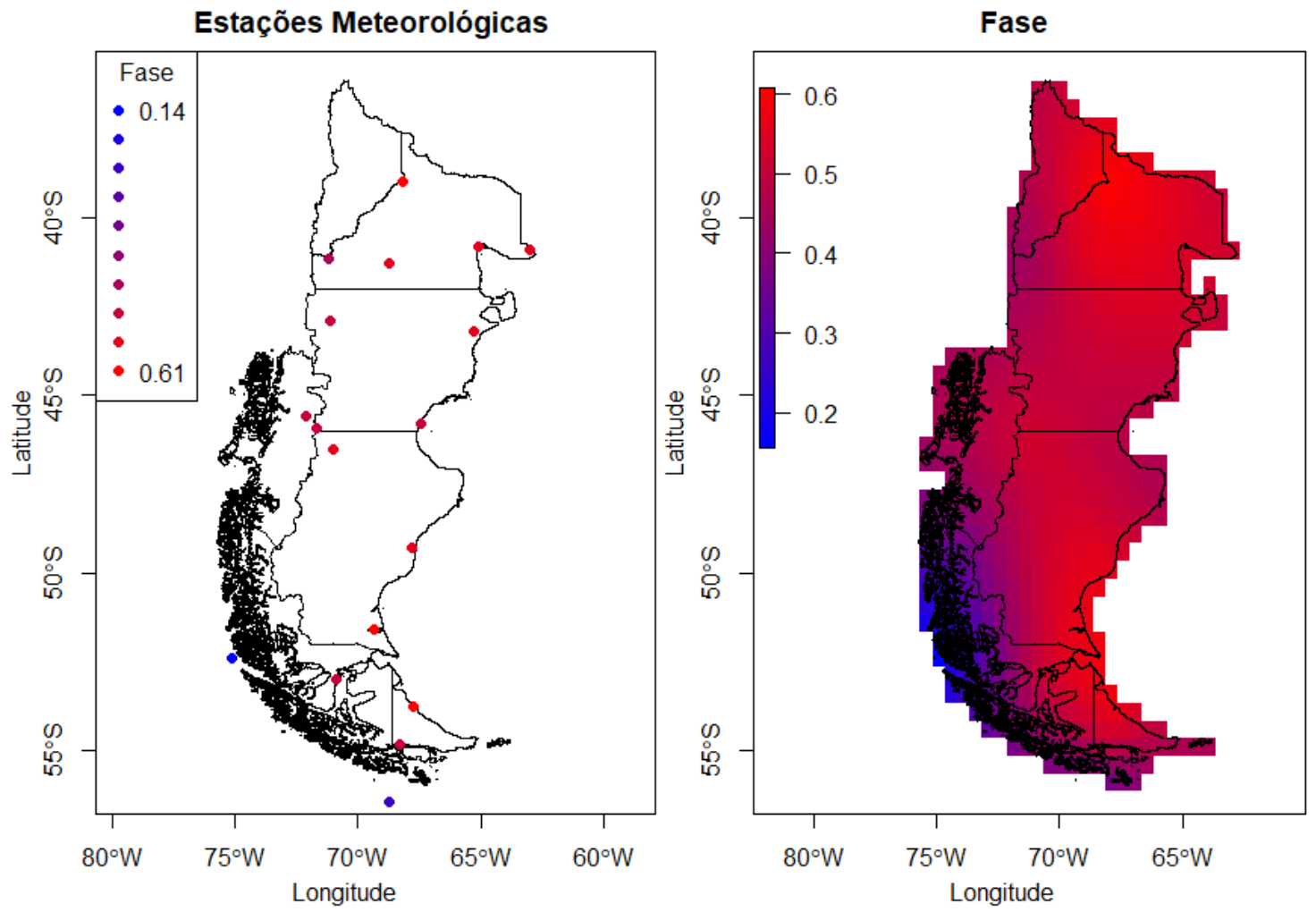

Figura 31 - Distribuição espacial da variável regionalizada fase e seu mapa krigado
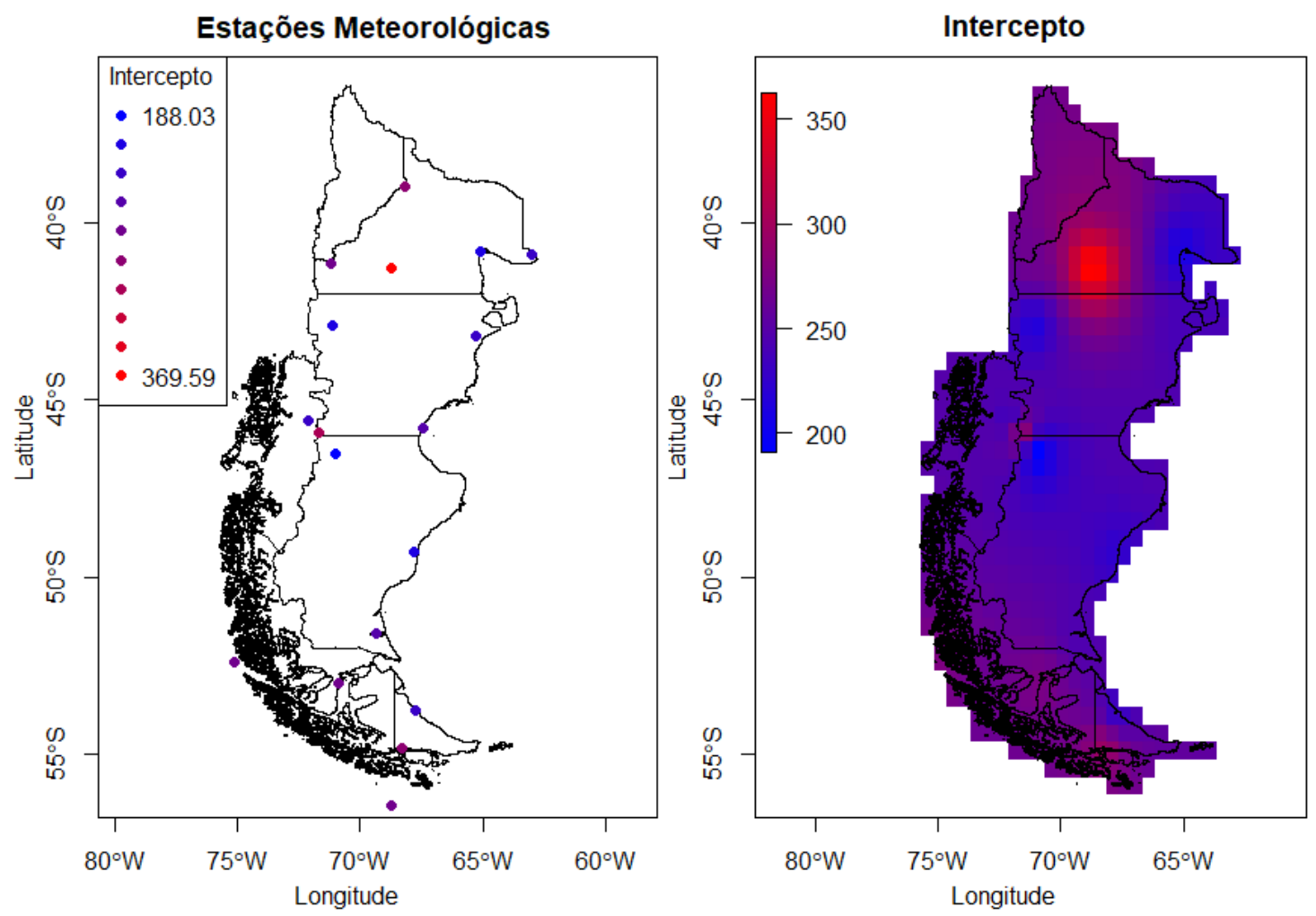

Figura 32 - Distribuição espacial da variável regionalizada intercepto e seu mapa interpolado 


\subsection{Variogramas Espaçotemporais}

O variograma espaçotemporal foi calculado para os dados brutos e os dois conjuntos de dados decompostos. A primeira etapa é escolher qual a classe de covariância espaçotemporal os dados pertencem, para isso De Iaco et al. (2016) e Cappello et al. (2018) demonstram como verificar a simetria, separabilidade, não separabilidade, modelo produto soma, produto integrado e outros. Cada tipo de classe de covariância tem um modelo que ajusta melhor a interação espaçotemporal dos dados. Os testes propostos por De Iaco et al. (2016) mostram que para este estudo, a hipótese nula de separabilidade não pode ser rejeitada com um nível de significância de 0,05 para as variáveis decompostas. Para os dados brutos os resultados foram inconclusivos, então decidiu-se utilizar o modelo do tipo separável. O modelo separável, também conhecido como modelo produto, é um dos modelos de variância espaçotemporal mais utilizado devido a sua simplicidade. Além disso, Gräler et al. (2016) demonstram as vantagens computacionais que este modelo tem. Montero et al. (2015) explicam que os modelos separáveis são os mais utilizados em aplicações geoestatísticas devido a sua alta eficiência computacional, ele é utilizado até mesmo em situações que sua hipótese não é justificada pela natureza dos dados analisados.

Os variogramas foram modelados de maneira interativa como pode ser observado na Figura 33, na Figura 34 e na Figura 35, para, respectivamente, o variograma dos dados brutos, com decomposição global e com decomposição local. As figuras mostram os variogramas experimentais espaçotemporais no lado esquerdo e os seus respectivos modelos no lado direito.

Os parâmetros dos modelos teóricos dos variogramas brutos (sem decomposição) e com decomposições global e local) estão na Tabela 3. Os variogramas experimentais foram analisados e foi notado que todos apresentam amplitude por volta de 8 graus no espaço e 1 ano no tempo. Além disso, o modelo exponencial é mais adequado para o variograma da decomposição global, enquanto o modelo esférico é mais adequado para os outros. Finalmente, os patamares foram escolhidos interativamente através testes exaustivos e um dos critérios para selecionar cada patamar foi analisar a Figura 36 que mostra a diferença do modelo com o variograma experimental. A diferença entre os modelos e o variograma experimental mostra que o ajuste foi melhor para os dados da decomposição local, com variação aproximada entre 2,5 e 1. Além disso, o variograma dos dados brutos mostra uma oscilação temporal bem demarcada pela sazonalidade da temperatura, esta sazonalidade é menor no método de decomposição global e quase nula na decomposição local. Este efeito oscilatório nos 
variogramas experimentais é conhecido como efeito furo nos dados espaciais (principalmente de mineração), ele ocorre quando diferentes padrões são intercalados (i.e., intercalação de duas rochas). No caso deste estudo, este comportamento indica que existe uma sazonalidade na informação de temperatura devido às estações do ano. Este efeito foi menor nos variogramas experimentais dos dados decompostos, pois foi possível modelar e eliminar parte ou totalmente as tendências e sazonalidades dos dados.

Tabela 3 - Parâmetros do ajuste do modelo teórico dos variogramas espaçotemporais

\begin{tabular}{ccccc}
\hline & & Bruto & Global & Local \\
\hline \multirow{4}{*}{ Modelo Espaçotemporal } & Produto & Produto & Produto \\
& Efeito pepita & 0 & 0 & 0 \\
& Patamar total & 20 & 15 & 2,2 \\
\multirow{4}{*}{ Espaço } & Patamar (\%total) & 1 & 1 & 0,6 \\
& Modelo & Esférico & Exponencial & Esférico \\
& Amplitude (Graus) & 8 & 8 & 8 \\
Tempo & Patamar (\%total) & 1 & 0,18 & 0,8 \\
& Modelo & Esférico & Exponencial & Esférico \\
& Amplitude (Dias) & 360 & 360 & 360 \\
\hline
\end{tabular}

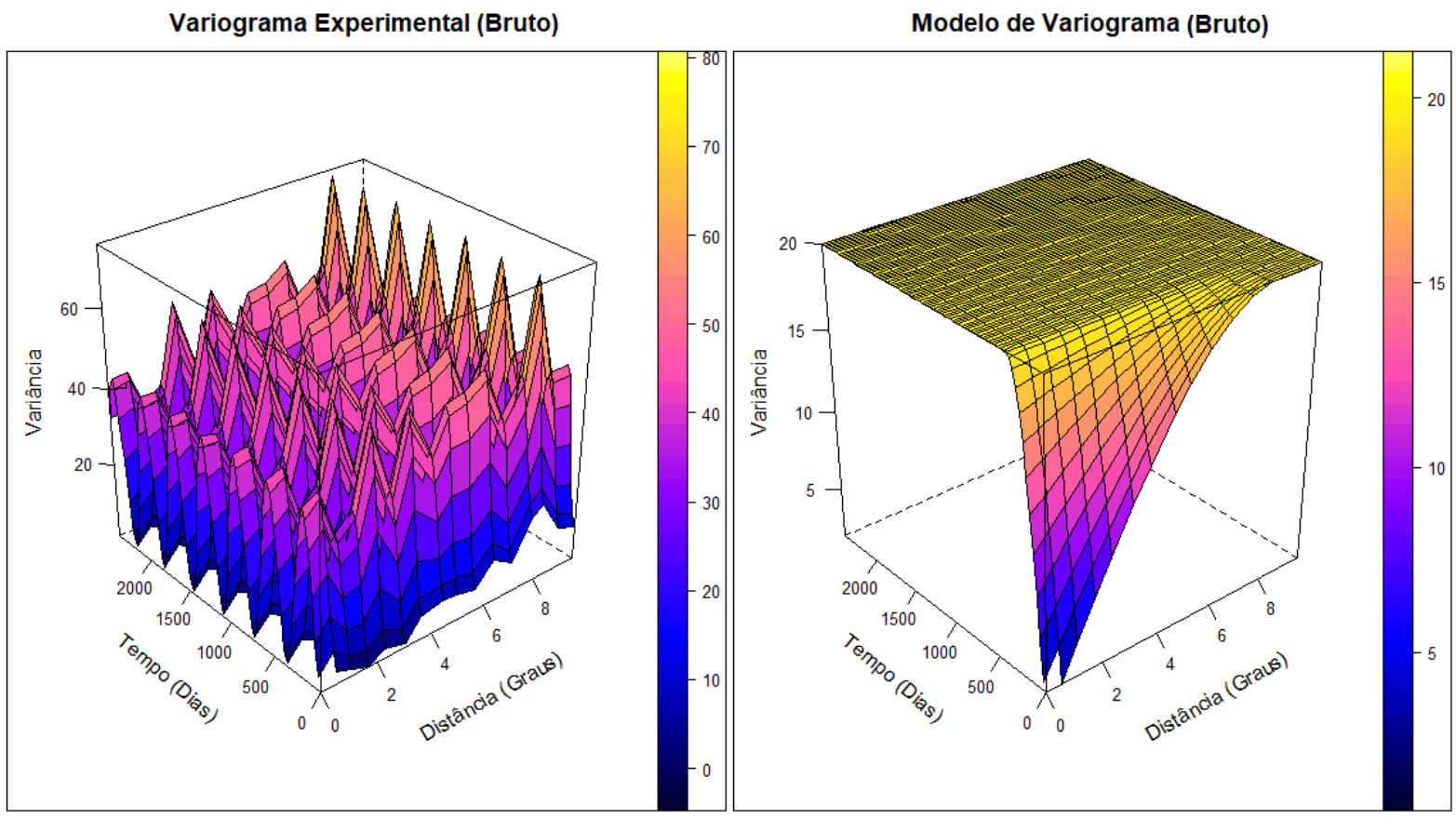

Figura 33 - Variograma espaçotemporal experimental e modelo teórico da variável temperatura brutos (sem decomposição) 


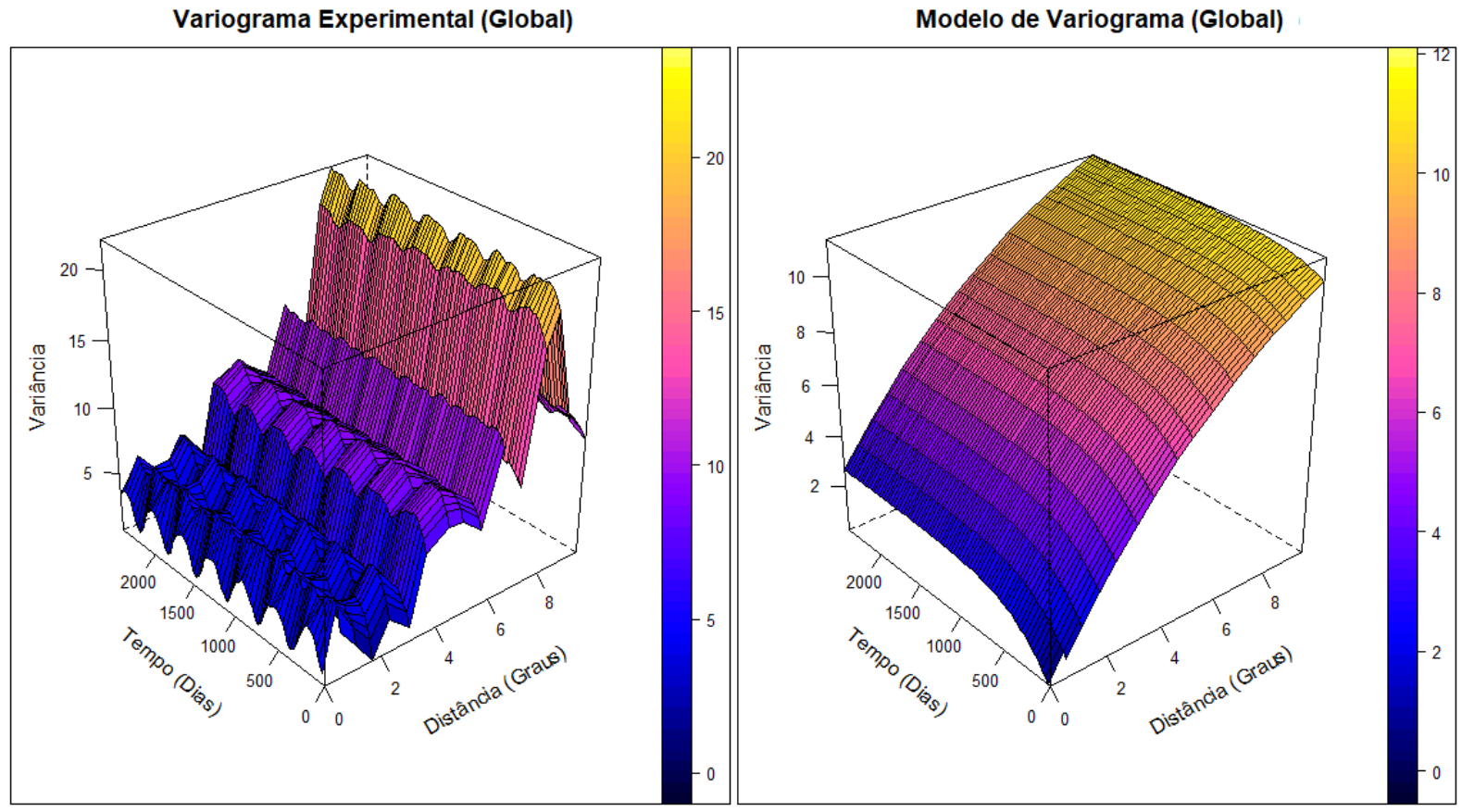

Figura 34 - Variograma espaçotemporal experimental e modelo teórico da variável temperatura com decomposição global

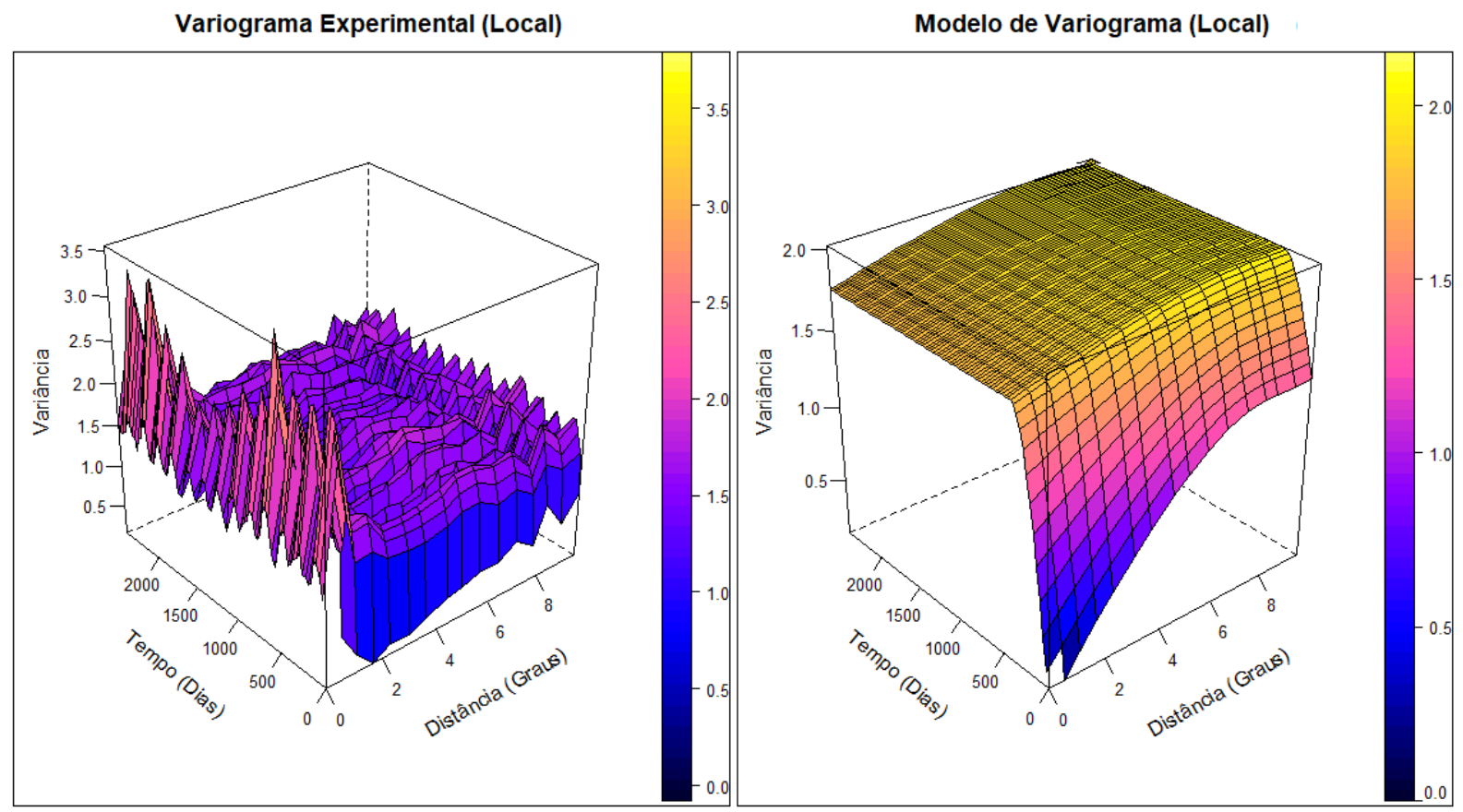

Figura 35 - Variograma espaçotemporal experimental e modelo teórico da variável temperatura com decomposição local 


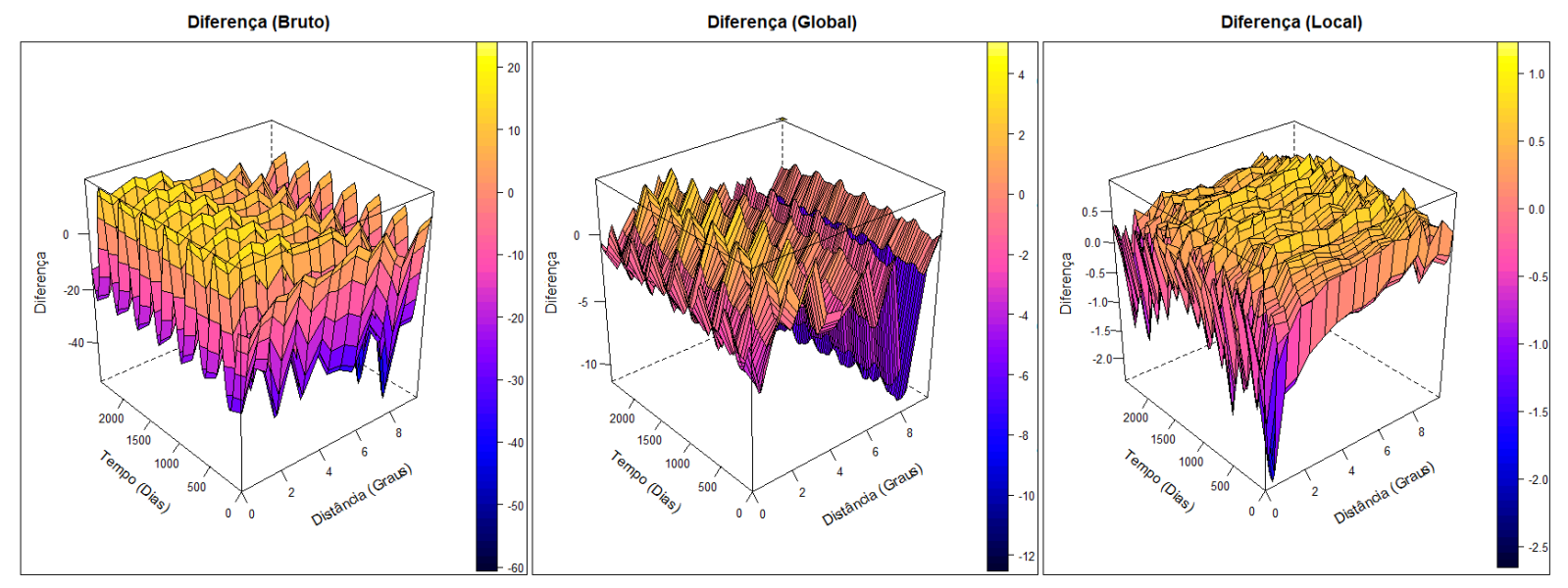

Figura 36 - Diferença do variograma espaçotemporal experimental e modelo teórico da variável temperatura dos dados brutos e decompostos (globalmente e localmente)

\subsection{Simulação e Resultados}

A simulação por bandas rotativas foi realizada com o objetivo de gerar realizações equiprováveis dos mapas de temperatura para todos os meses do ano de 2017. Para isso, foi utilizada a mesma malha dos mapas dos parâmetros de séries temporais, o tamanho da célula é de $0,5^{\circ}$ em latitude e longitude. A simulação utilizou uma vizinhança de busca global, foram feitas 100 realizações e foram aplicadas 500 bandas rotativas para garantir que os resultados não estão gerando artefatos.

Portanto, os resultados da simulação são 100 mapas da temperatura de cada mês do ano de 2017. Para cada mês, os 100 resultados são equiprováveis, assim pode-se trabalhar com a distribuição de valores em cada pixel simulado. A Tabela 4 compara as estatísticas descritivas dos resultados médios, $\mathrm{P}(5)$ e $\mathrm{P}(95)$ para os dados brutos e com decomposições global e local. Os resultados podem ser comparados com a Tabela 1, que mostra os valores das estações meteorológicas (período de calibração e de controle) e LST. Esta imagem de satélite (LST) tem resolução original de $0,05^{\circ}$ em latitude e longitude e foi ajustada por média aritmética para ser apresentada na mesma escala dos resultados. Assim, é possível ver que as estatísticas médias das simulações feitas com decomposição (local ou global) são as que mais se aproximam dos dados das estações, tanto no período de calibração, quanto de controle. De modo geral, as estatísticas dos mapas previstos mostraram distribuições semelhantes àquelas encontradas nos dados das estações meteorológicas. 
Tabela 4 - Estatística da média P(5) e P(95) da temperatura simulada (em Kelvin) com e sem decomposição (global e local)

\begin{tabular}{cccccccc}
\hline & Min. & $\mathrm{P}(25)$ & $\mathrm{P}(50)$ & Média & $\mathrm{P}(75)$ & Máx. & $\begin{array}{c}\text { Desvio } \\
\text { Padrão }\end{array}$ \\
\hline Brutos (Média) & 281,7 & 283,8 & 284,6 & 285,7 & 287,0 & 295,9 & 2,83 \\
Brutos (P(5)) & 270,0 & 275,1 & 276,4 & 277,1 & 278,4 & 293,2 & 3,19 \\
Brutos (P(95)) & 284,4 & 291,5 & 292,8 & 293,6 & 295,0 & 307,4 & 3,10 \\
Global (Média) & 274,7 & 279,3 & 283,6 & 283,4 & 287,0 & 296,7 & 4,84 \\
Global (P(5)) & 272,7 & 277,5 & 281,8 & 281,7 & 285,4 & 296,2 & 4,91 \\
Global (P(95)) & 276,5 & 281,4 & 285,5 & 285,5 & 289,2 & 297,9 & 4,76 \\
Local (Média) & 274,1 & 279,0 & 282,7 & 283,2 & 287,0 & 297,8 & 5,22 \\
Local (P(5)) & 272,1 & 277,2 & 280,9 & 281,5 & 285,3 & 297,4 & 5,29 \\
Local (P(95)) & 276,2 & 281,1 & 284,7 & 285,3 & 289,1 & 298,7 & 5,15 \\
\hline
\end{tabular}

As estatísticas médias são semelhantes aos valores esperados, porém o mapa da previsão feito para dezembro de 2017, ou seja, 1 ano após os dados mais recentes de calibração (dezembro de 2016), mostram que os valores em cada pixel tendem a média global dos dados para a estimativa com dados brutos e com decomposição global. Isto fica claro na Figura 37, na qual os mapas médios dos resultados de simulação para os 3 métodos no mês de dezembro de 2017 são comparados. Este resultado indica que a geoestatística espaçotemporal com deriva externa temporal local pode simular valores a longo prazo, sem que os valores tendam para a média como ocorreu com os outros resultados.
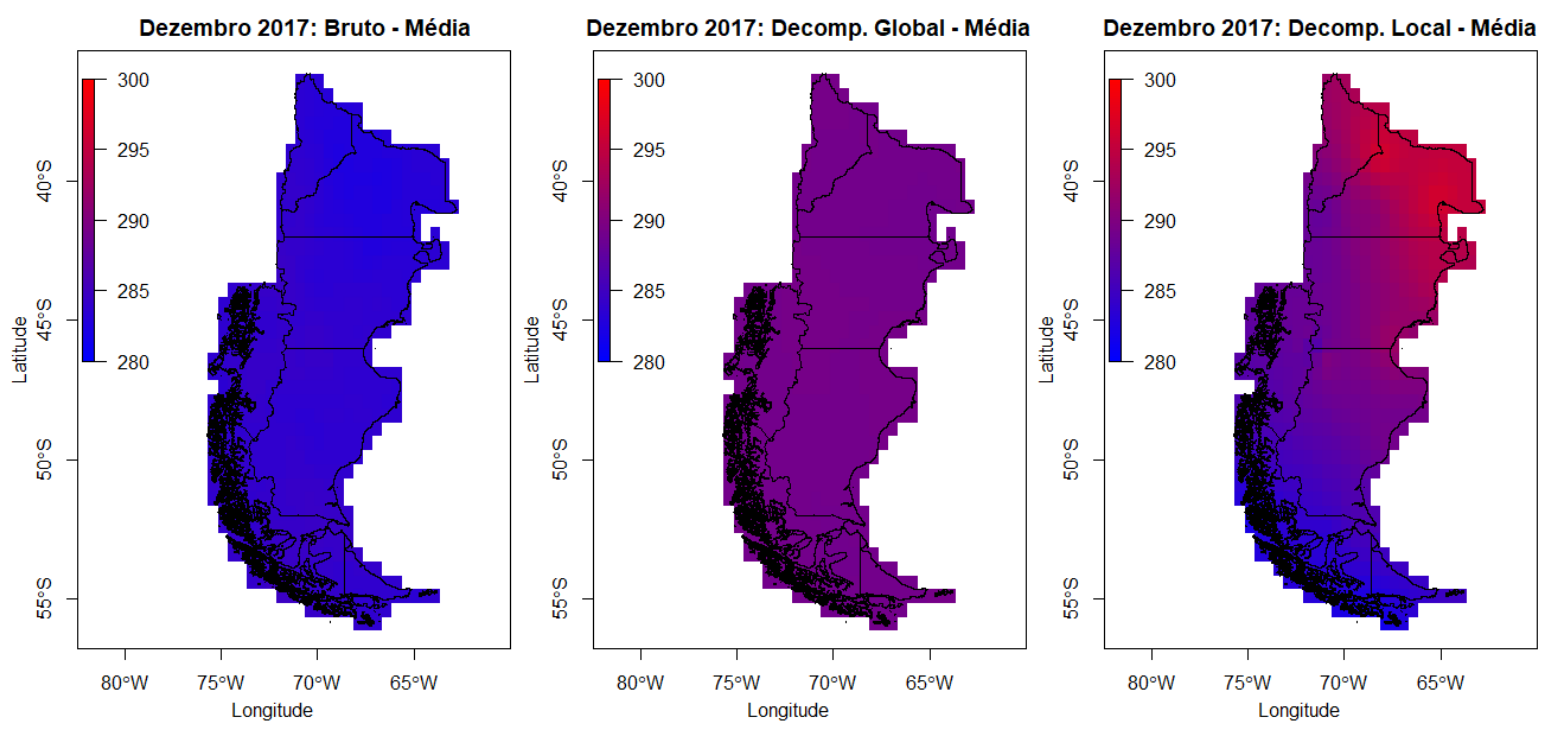

Figura 37 - Mapa da média da simulação feita com os dados brutos e decompostos (globalmente e localmente) para a previsão do $12^{\circ}$ mês 
Quando analisamos a variância dos pixels da simulação, fica óbvia a necessidade da utilização de algum método de decomposição. A Figura 38 mostra o mapa da variância dos resultados para o mês de dezembro de 2017. A escala da variância dos dados brutos é de 10 a 30 , enquanto dos resultados que utilizaram a decomposição é entre 0 e 2 . Os padrões espaciais dos 3 resultados são diferentes, o que mostra que cada dado simulado tem sua própria particularidade espacial. Quando comparado com a Figura 20, é possível observar que a variância não depende tanto da localização dos pontos amostrados, mas sim de quão heterogênea é a região.
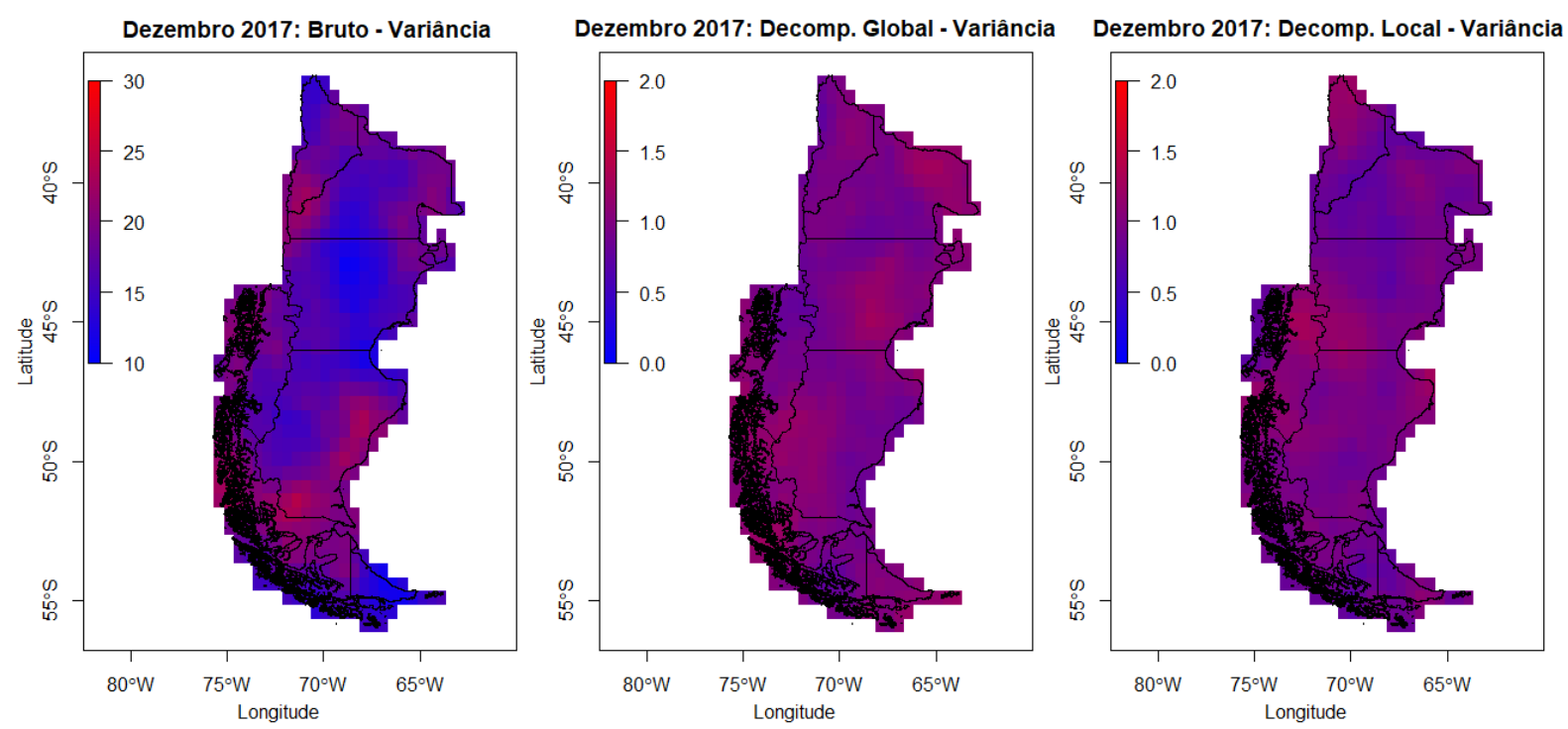

Figura 38 - Mapa da variância da simulação feita com os dados brutos e decompostos (globalmente e localmente) para a previsão do $12^{\circ}$ mês

A análise da média (Figura 37) e da variância (Figura 38) indica que existe oscilação nos valores das realizações do mês de dezembro. A maior amplitude desta distribuição de valores está nos dados brutos (maior variância em cada pixel) e isto aparece nos mapas de $\mathrm{P}(5)$ e P(95), respectivamente a Figura 39 e a Figura 40. A diferença entre o $\mathrm{P}(5)$ e $\mathrm{P}(95)$ dos dados brutos é nítida, a coloração azulada do $\mathrm{P}(5)$ indica que muitas células apresentam valores baixos, enquanto a coloração avermelhada do $\mathrm{P}(95)$ indica muitos valores próximos aos valores máximos. Com a mesma análise, é possível ver que a comparação dos resultados do P(5) e $\mathrm{P}(95)$ são visualmente muito parecidos, este era o resultado esperado para regiões com variância de simulação baixa. Uma característica comum, tanto nos mapas $\mathrm{P}(5)$ e $\mathrm{P}(95)$ para os dados brutos, quanto para os dados decompostos globalmente é a variação de cores, ou seja, apesar da 
alta variância (localmente e espacialmente) dos dados brutos, os mapas extremos são quase tão monocromáticos quanto os valores da média.
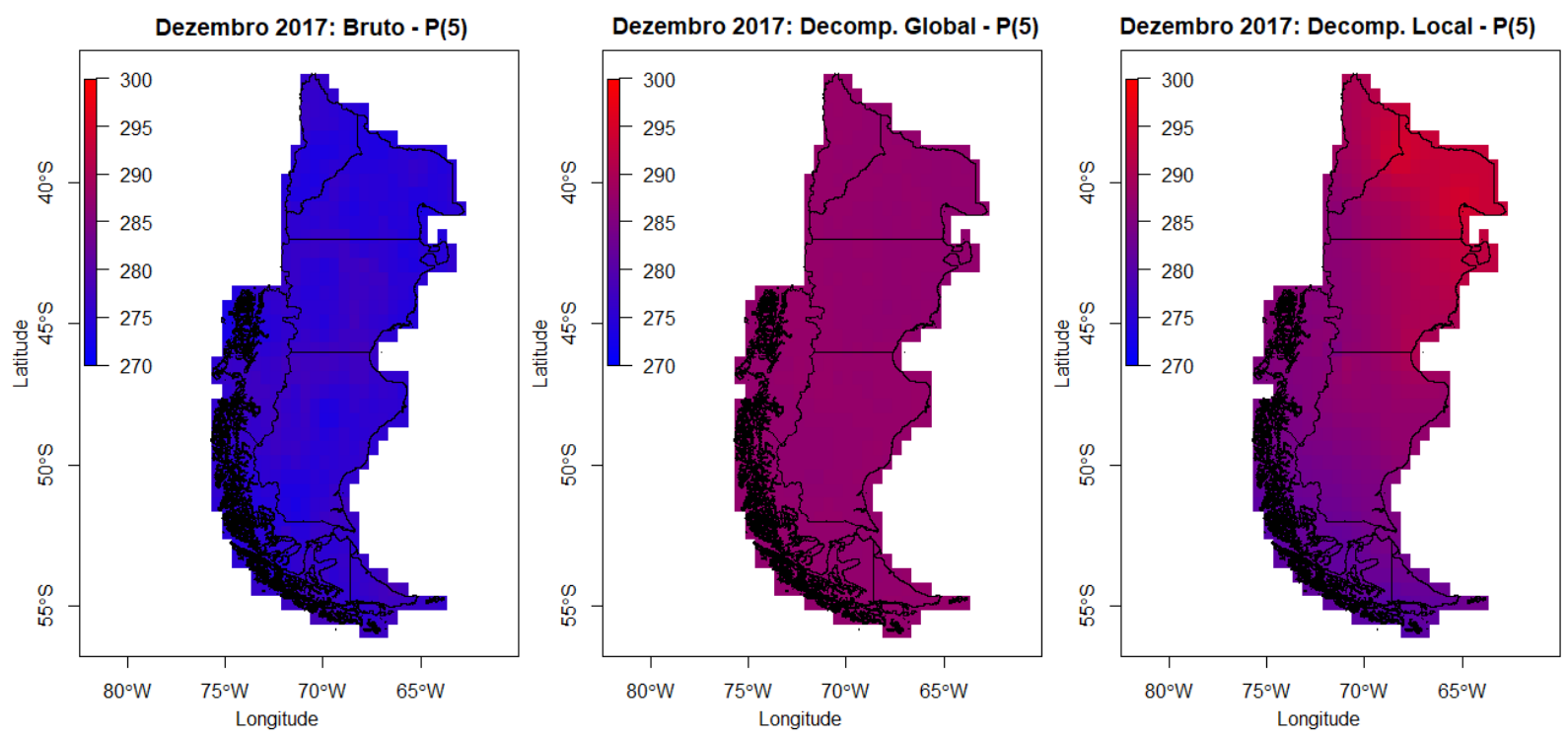

Figura 39 - Mapa do $\mathrm{P}(5)$ da simulação feita com os dados brutos e decompostos (globalmente e localmente) para a previsão do $12^{\circ}$ mês
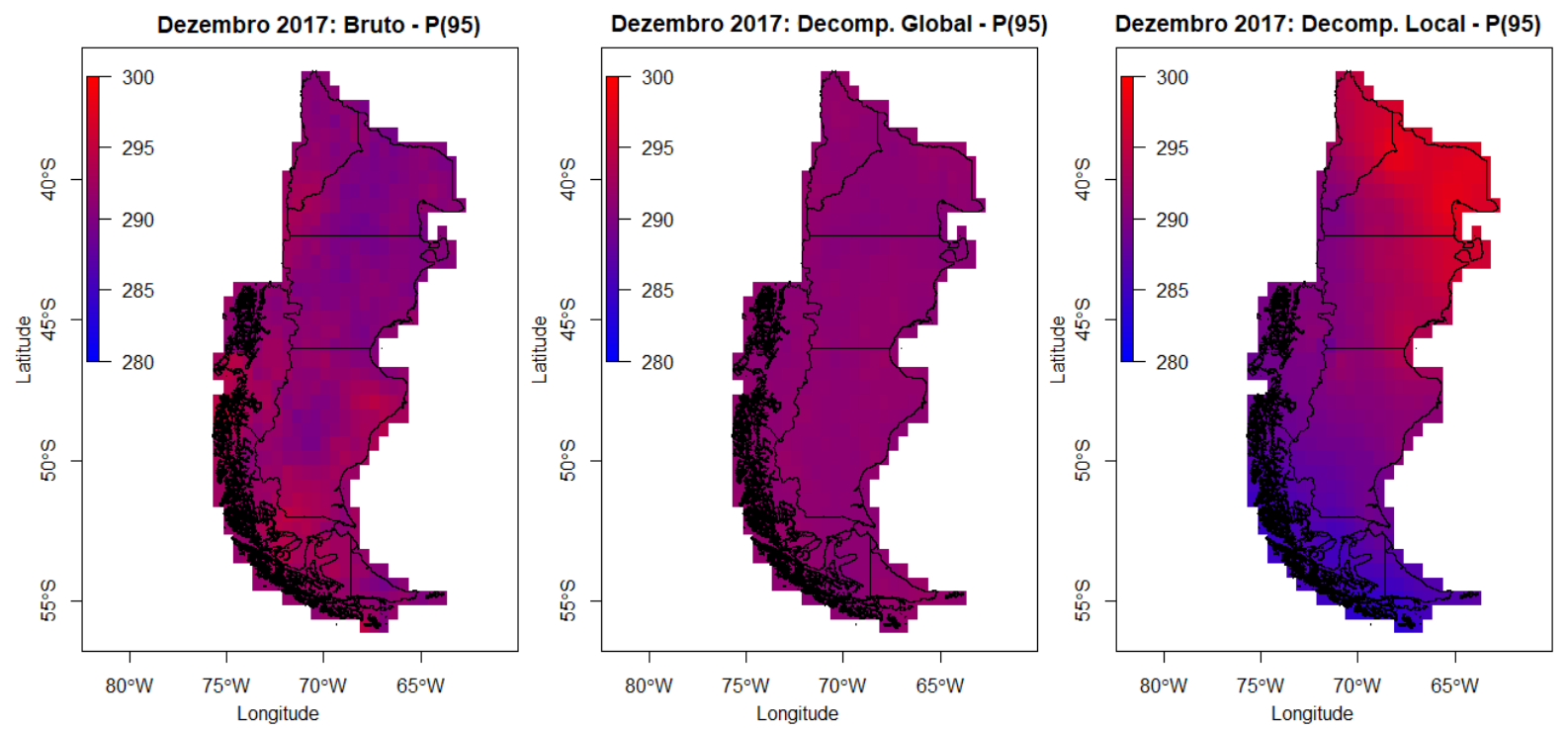

Figura 40 - Mapa do P(95) simulação feita com os dados brutos e decompostos (globalmente e localmente) para a previsão do $12^{\circ}$ mês 
Além da variância e da comparação dos valores extremos, é possível comparar os resultados calculando a raiz do erro quadrático médio (RMSE). Esta análise avalia a acurácia dos métodos, ou seja, ela quantifica o erro em cada local do mapa. Assim, pode-se comparar os resultados dos 3 métodos para escolher qual deles é mais acurado na previsão de temperatura. Porém, a análise necessita do conhecimento dos valores reais de temperatura em cada pixel estudado, por isso o produto LST (Wan et al., 2015) da imagem de satélite foi considerado como os valores verdadeiros. Assim, a RMSE (Eq. 56) foi calculada para cada pixel no mapa de temperatura (Figura 41). Fica evidente a necessidade da decomposição temporal, pois os RMSE dos dados brutos apresentam um mapa com valores mais elevados do que os mapas de RMSE das variáveis decompostas. Dentre os métodos de decomposição, é possível constatar que a decomposição local tem valores de RMSE menores.

A região da cordilheira dos Andes apresenta erro mais elevado nos 3 métodos, isto ocorre devido à falta de informação na região. Sua altitude pode provocar diferenças climáticas não observada nas regiões em que existem estações meteorológicas, causando um problema para a previsão com qualquer um dos métodos testados neste estudo. Os métodos geoestatísticos e séries temporais consideram que a amostragem é adequada, portanto eles não conseguem estimar resultados fora de seus padrões.
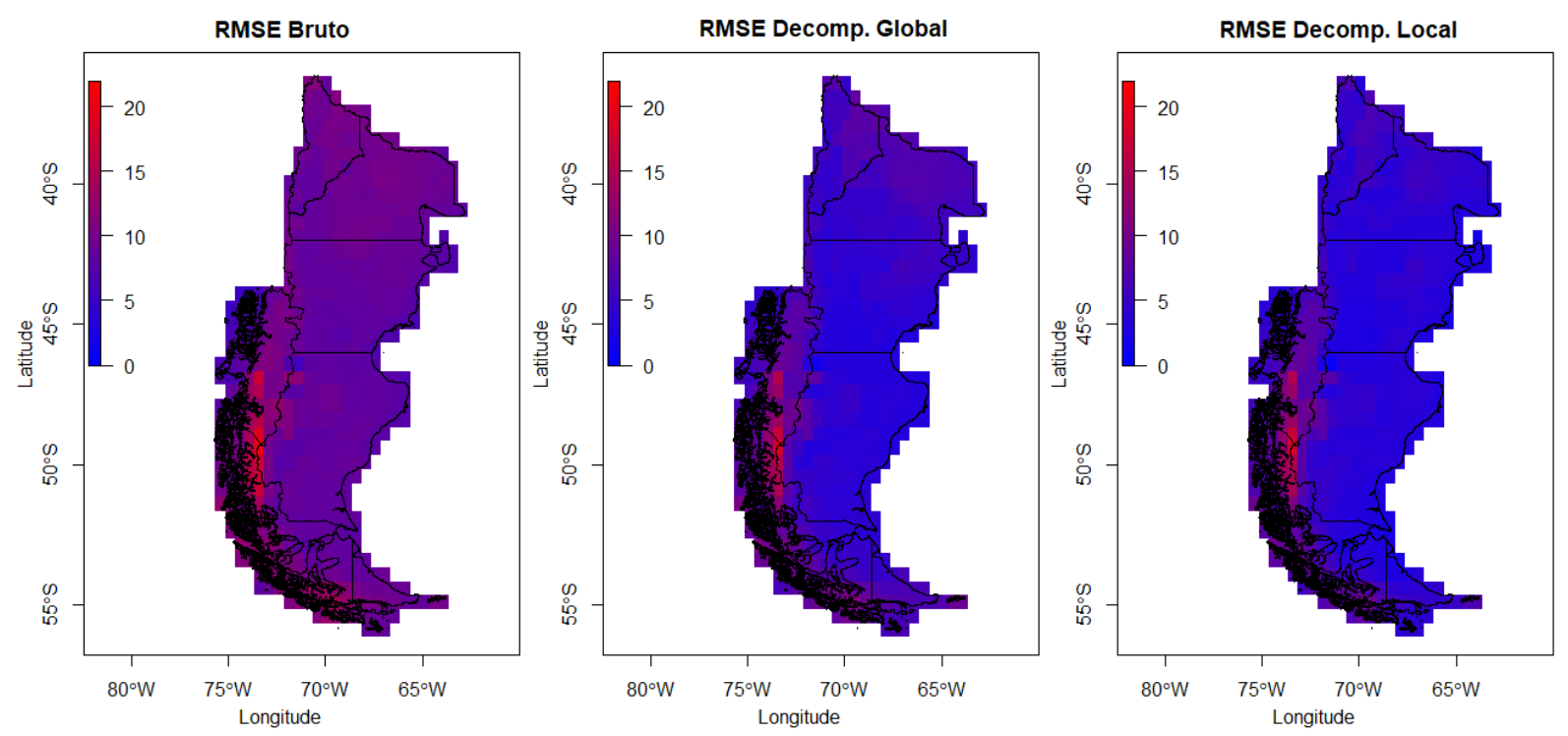

Figura 41 - Mapa do RMSE da simulação feita com os dados brutos e decompostos (globalmente e localmente) 
A Figura 42 mostra a distribuição acumulada dos valores médios das simulações e compara com os valores das estações de calibração. Esta comparação mostra a aderência das distribuições em relação aos dados das estações meteorológicas de calibração, ou seja, quão próxima é a distribuição dos dados em relação aos dados amostrais e à distribuição considerada verdadeira (LST). Destaca-se que a simulação com os dados brutos (linha verde) suavizou muito os resultados, por isso sua linha é extremamente verticalizada. As duas simulações com valores decompostos tiveram distribuições muito próximas com a distribuição dos dados de temperatura das estações meteorológicas, sendo que a simulação com deriva externa temporal local foi a mais aderente aos dados das estações meteorológicas. Por fim, a distribuição da temperatura LST é a menos suavizada de todas, ela é uma variável que cobre toda a área e é mais próxima da realidade, ela captura todos os valores locais de temperatura e, por isso, existe muito mais informação nesta distribuição.

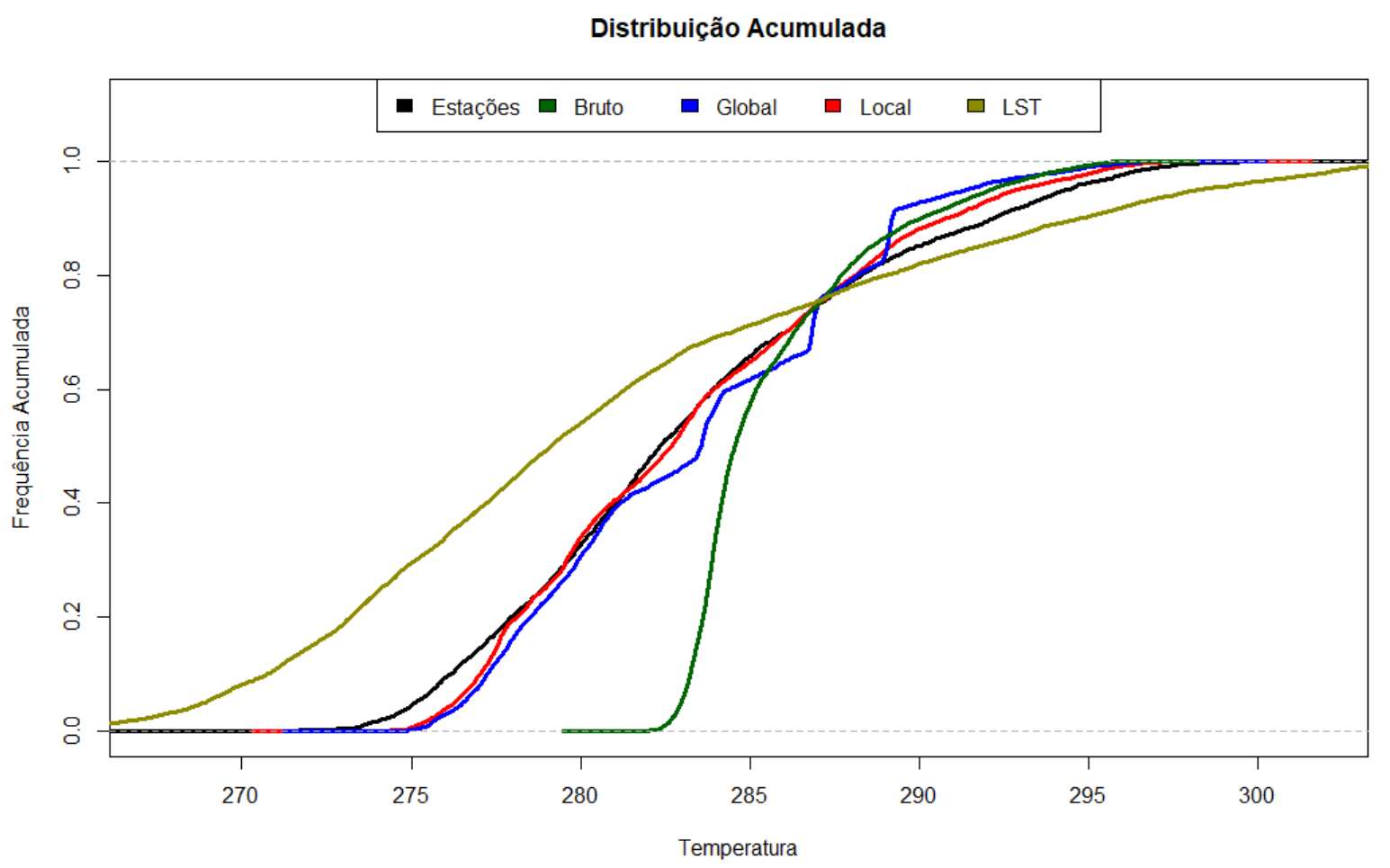

Figura 42 - Distribuição acumulada dos dados da média de temperatura simulados com e sem decomposição (global e local) comparados com os dados das estações meteorológicas de calibração e imagem de satélite (LST) 
A fim de comparar a dispersão dos resultados das simulações, foi calculada a distribuição acumulada do $\mathrm{P}(5)$ e $\mathrm{P}(95)$. Mostra-se na Figura 43 que os 3 métodos simulados têm dispersões ao redor dos dados das estações meteorológicas utilizadas na simulação. $\mathrm{O}$ método de simulação com os dados brutos teve a maior dispersão de todas, enquanto os métodos com decomposição apresentaram menor dispersão ao redor dos dados. O método de decomposição local apresenta resultados com uma distribuição bem estável ao redor da curva das estações. A curva da simulação com decomposição global apresenta diversas instabilidades. Provavelmente, isto ocorre devido a decomposição global não ser aderente a todas as séries temporais das estações meteorológicas. Nesta figura é possível observar que a alta variabilidade dos resultados sem decomposição fez com que quase toda a distribuição do LST ficasse dentro de seus valores extremos $(\mathrm{P}(5)$ e $\mathrm{P}(95))$. Este resultado é ilusório, pois é notório que a distribuição acumulada desta simulação não adere aos dados das estações meteorológicas tampouco ao LST.

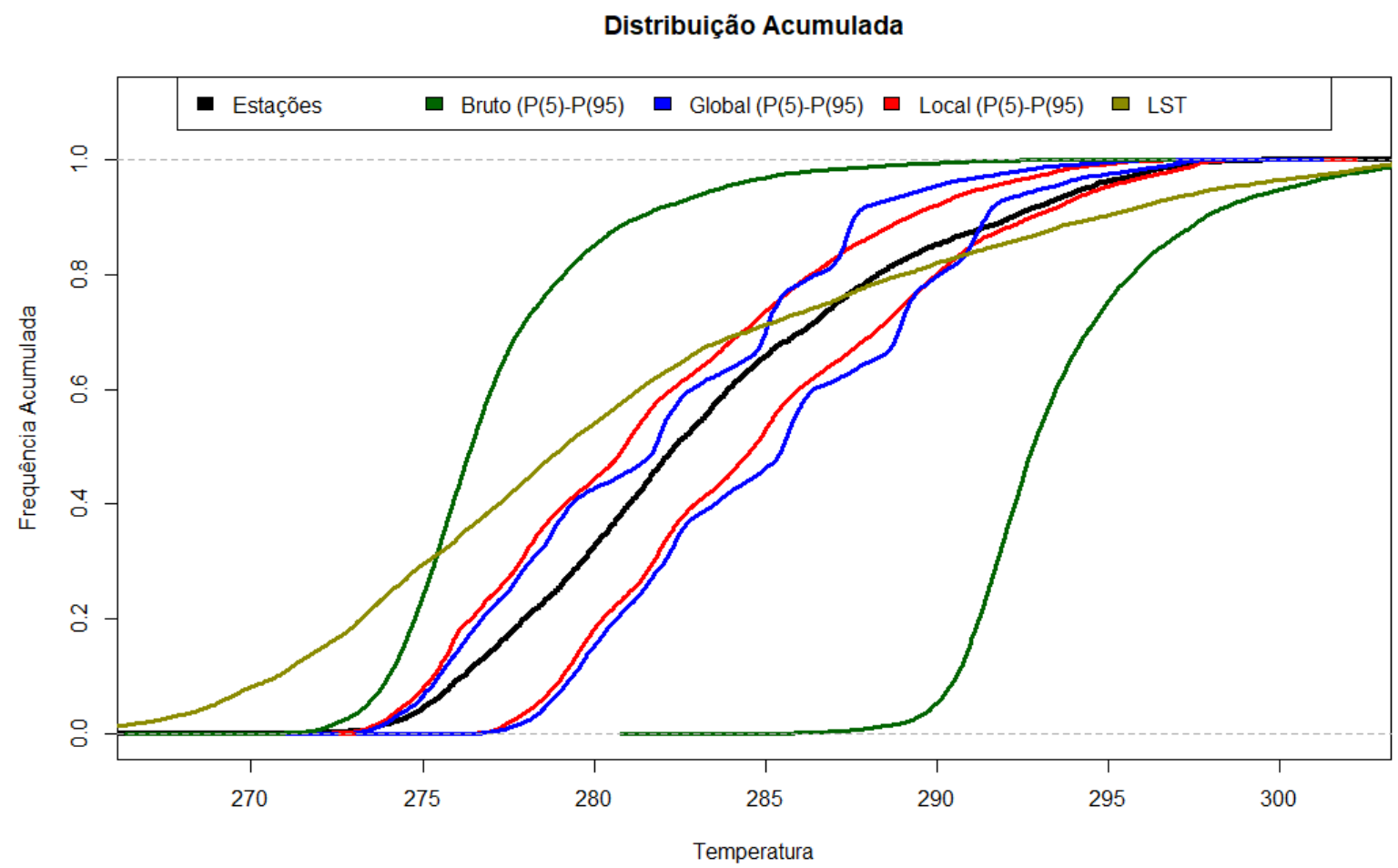

Figura 43 - Distribuição acumulada dos dados de $\mathrm{P}(5)$ e $\mathrm{P}(95)$ de temperatura simulados com e sem decomposição (global e local) comparados com os dados das estações meteorológicas de calibração e imagem de satélite (LST) 
A variável espaçotemporal permite não apenas a observação de sua distribuição espacial (mapa) e de valores (distribuição acumulada), mas também é possível ver sua distribuição no tempo. Sendo assim, compara-se na Figura 44 os valores médios, $\mathrm{P}(5)$ e $\mathrm{P}(95)$ das simulações mensais. A Figura 44A mostra que as médias mensais das simulações com decomposição são aderentes a média dos dados das estações meteorológicas. Além disso, o resultado da simulação sem decomposição tende a média global dos dados e não acompanha sazonalidade temporal observada. Já a Figura 44 B, C e D mostram os resultados de cada método, com sua média e dispersão ( $\mathrm{P}(5)$ e $\mathrm{P}(95))$. Mais uma vez, os resultados médios de ambos métodos de decomposição aderem aos resultados e suas dispersões são baixas e semelhantes.

Além disso, é possível ver que a falta de estações meteorológicas elevou a média de temperatura nos meses de inverno, pois os valores das estações são maiores que os valores observados pelo LST e os valores de temperatura nos meses de verão foram bem próximos.

Como já foi observado na Figura 37, os resultados podem tender a média global. Então a média observada na Figura 44 pode ser espacialmente suavizada, ou seja, não representar adequadamente a distribuição espacial dos dados, pois tende ao valor médio da região. A fim de ilustrar graficamente a variabilidade dos mapas mensais, a Figura 45 mostra o desvio padrão para cada mês em estudo. Assim, observa-se que as simulações sem decomposição e com decomposição global tendem a um valor médio global e seu desvio padrão tende a zero, pois não há mais distribuição espacial de valores (apenas um valor médio). Este resultado mostra suavização extrema (corroborado pelos dois primeiros mapas da Figura 37 que são basicamente monocromáticos). Enquanto o desvio padrão dos mapas feitos com a aplicação da geoestatística espaçotemporal com deriva externa temporal local é mais coerente com o desvio padrão dos dados das estações meteorológicas no período de controle. 
Temperatura

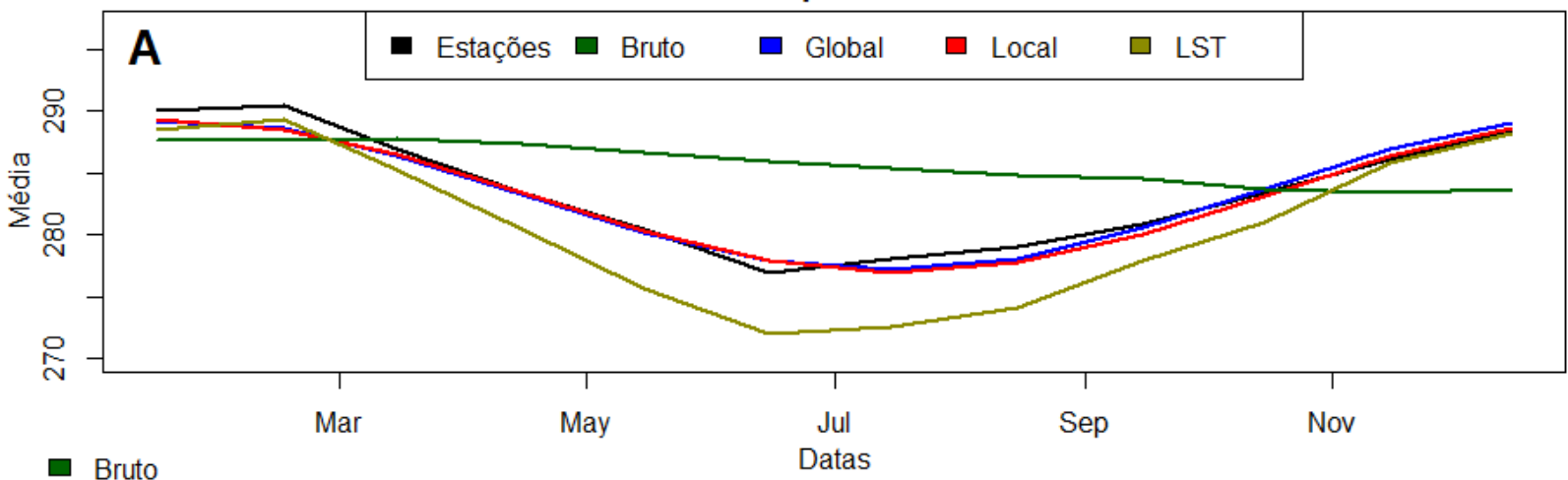

Temperatura

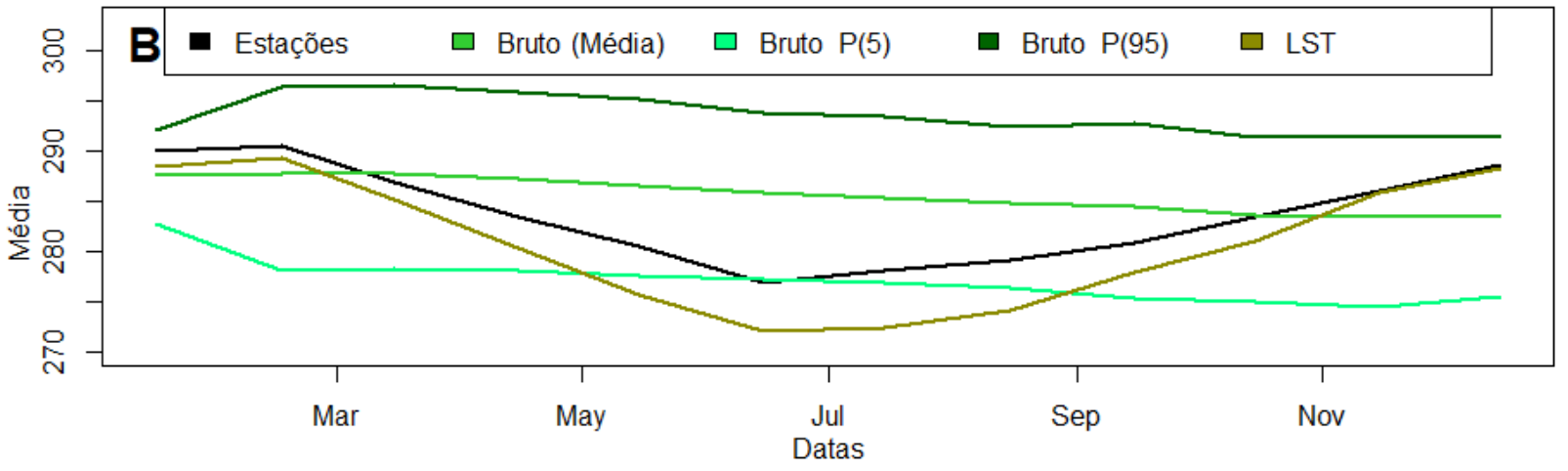

Temperatura

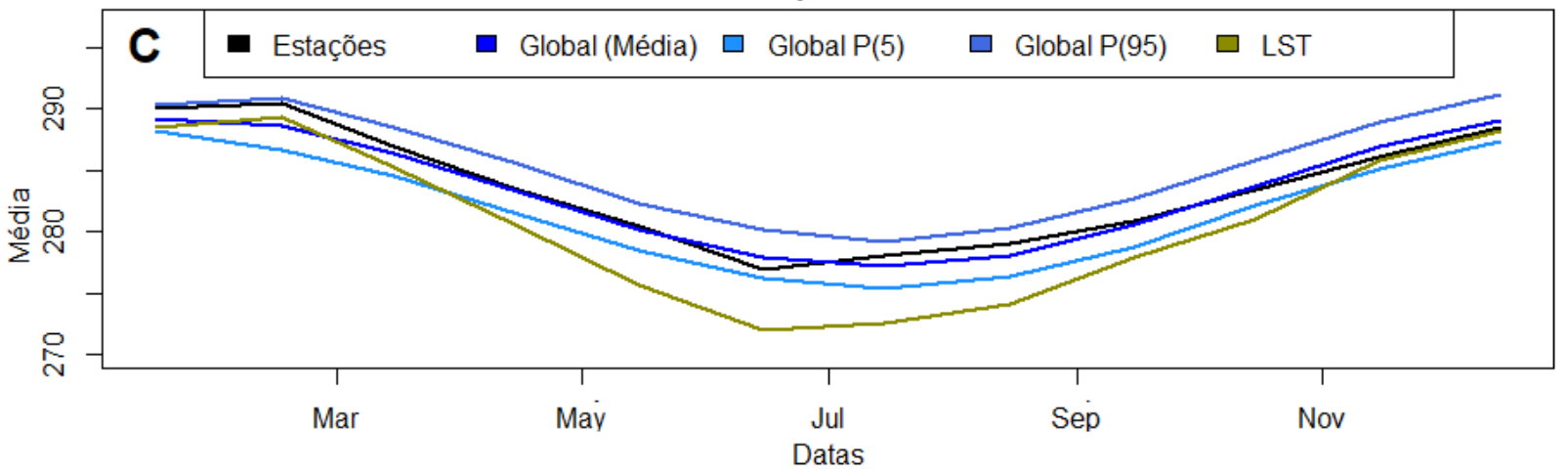

Temperatura

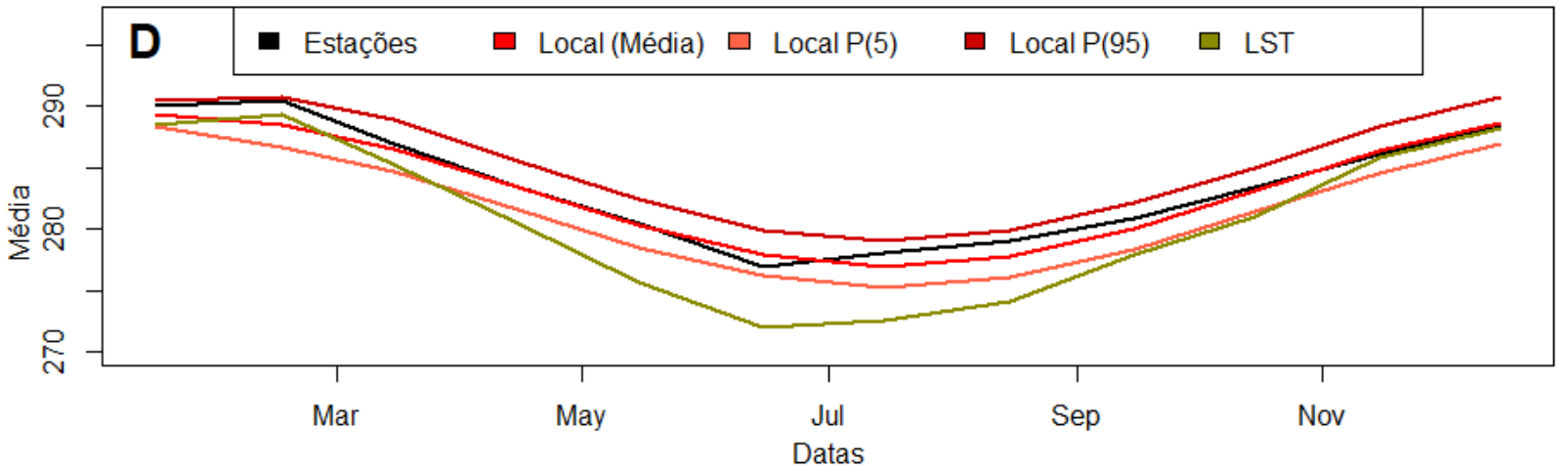

Figura 44 - Comparação mensal dos dados das estações meteorológicas de controle e imagem de satélite (LST) com as estatísticas (média, $\mathrm{P}(5)$ e $\mathrm{P}(95)$ dos valores de temperatura simulados com e sem decomposição (global e local) 
Temperatura

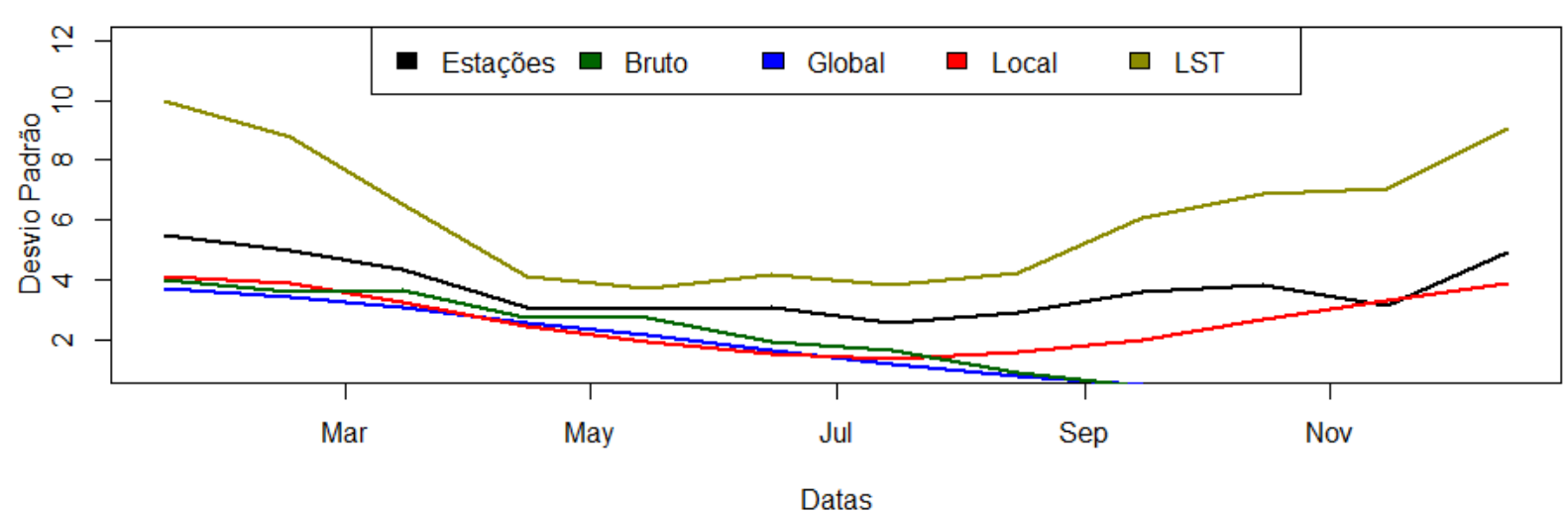

Figura 45 - Comparação mensal do desvio padrão dos dados das estações meteorológicas de controle e imagem de satélite (LST) com o desvio padrão dos valores médios de temperatura simulados com e sem decomposição (global e local)

A última avaliação dos resultados neste estudo é a comparação dos resultados com os erros mensais, ou seja, calcular a RMSE de cada mês em todos os métodos, o gráfico com este erro pode ser observado na Figura 46. Desta maneira é possível ver como a RMSE da simulação com os dados brutos é expressivamente alta em alguns meses. E que os métodos de decomposição têm resultados semelhantes até o mês de agosto, quando a decomposição global começou apresentar erros cada vez maiores. Além disso, existe um erro (RMSE = 2,95) das estações meteorológicas no período de controle quando comparado com os valores do LST. Sendo assim, este é um erro intrínseco aos dados e dificilmente um método de simulação apresente erros menores que esse. O valor médio das simulações dos dados brutos, com decomposições global e local tem RMSE de 8,97, 6,04 e 5,28, respectivamente. Para os casos extremos $(\mathrm{P}(5)$ e $\mathrm{P}(95))$ os valores a RMSE são respectivamente, 8,19 e 14,49 para a simulação dos dados brutos, 5,42 e 7,23 para a simulação dos dados decompostos globalmente e 4,73 e 6,47 para a simulação dos dados decompostos localmente. 
RMSE no Tempo

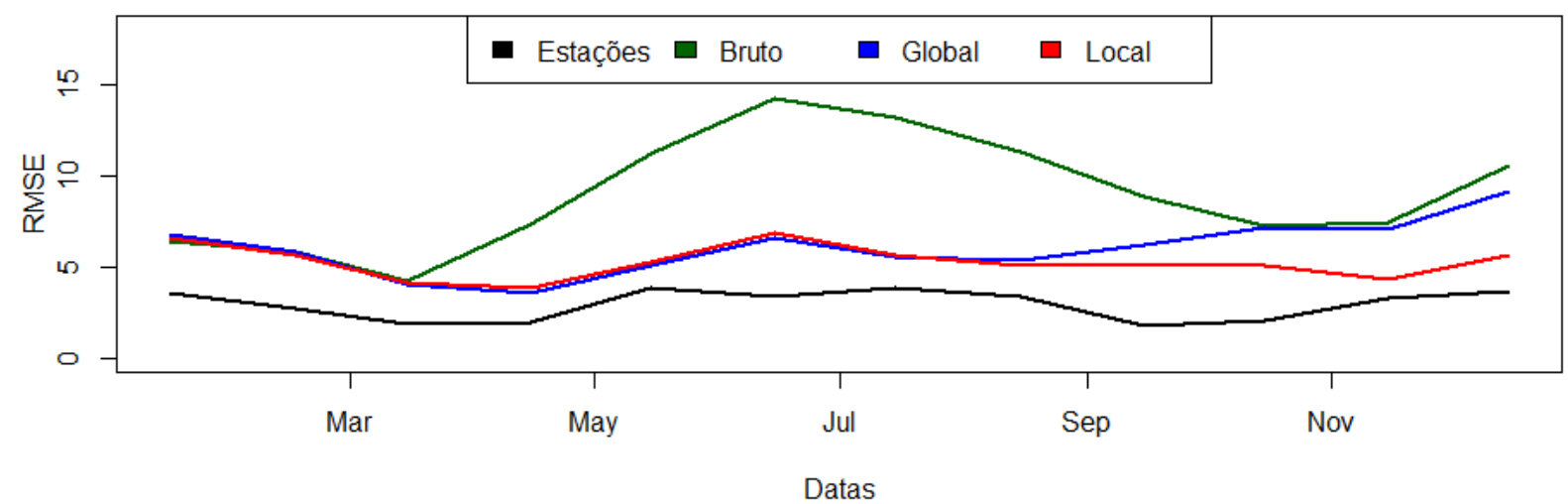

Figura 46 - Comparação mensal do RMSE dos dados das estações meteorológicas de controle e imagem de satélite (LST) com os valores do RMSE da média das simulações com e sem decomposição (global e local)

A Tabela 5 mostra o sumário das validações entre os três métodos aplicados. Na tabela fica evidente a eficácia do método de decomposição local. A precisão foi analisada considerando a gama de possibilidades geradas pela simulação (Figura 43 e Figura 44), a exatidão calculada com base no RMSE total e a suavização foi observada na preservação dos padrões espaciais dos mapas futuros (Figura 37).

Tabela 5 - Sumário das validações

\begin{tabular}{cccc}
\hline & \multicolumn{2}{c}{ Geoestatística espaçotemporal } & \\
& Dados Brutos & Decomposição Global & Decomposição Local \\
\hline Precisão & Baixa & Alta & Alta \\
Exatidão & Baixa & Média & Alta \\
Suavização & Alta & Alta & Baixa \\
\hline
\end{tabular}




\section{CONCLUSÃO E TRABALHOS FUTUROS}

O método proposto permite fazer mapas de previsões para qualquer data, seja em períodos anteriores ao amostrado, no período amostrado ou no futuro. Entretanto, o pesquisador deve assumir que a tendência e sazonalidade do período amostrado possa ser assumida válida para o período estudado. Por isso, não é recomendado simular um futuro ou passado muito distante, pois os padrões, provavelmente, são diferentes.

O estudo de caso mostrou que o método de simulação espaçotemporal com deriva externa temporal local consegue gerar mapas de previsão futura com mais precisão (menor variabilidade de resultados simulados) e exatidão (baixo erro) que os métodos de geoestatística espaçotemporal sem deriva e com deriva externa temporal global. A decomposição local de cada série temporal funciona perfeitamente e a interpolação das novas variáveis regionalizadas (parâmetros das séries temporais) pode ser feita tanto por krigagem ordinária, quanto interpolação não geoestatística (e.g. inverso da distância).

A comparação dos métodos em uma região crítica (baixa informação para a vasta área da Patagônia) mostrou a robustez do método de decomposição local que funcionou muito bem e quando comparado com os dados da imagem de satélite, este método manteve o erro (RMSE) mais baixos que os outros. Isto prova que o método tem maior exatidão em situações de baixa informação. As estatísticas mostram que a maneira mais correta de fazer um mapa de previsão futura é com a simulação espaçotemporal com deriva externa temporal local, pois a partir de um determinado período, com os outros métodos testados (krigagem espaçotemporal sem decomposição temporal e com decomposição global) a previsão tende a um mapa de valor médio. Estatisticamente isto é observado na Figura 45, onde mostra a variância dos resultados mensais tenderem a zero, ou seja, todos os pontos do mapa tendem a serem iguais. A suavização observada nestes métodos não acontece na deriva local, pois ela reproduziu o comportamento do desvio padrão mensal encontrados nas estatísticas das estações de controle mesmo depois de 12 meses de previsão.

Neste estudo de caso, observou-se a situação em que a variável com covariância de classe separável tem a componente temporal bem modelada, restando basicamente o ruído aleatório. Neste caso de separabilidade, em que é possível modelar bem as oscilações temporais, as análises de séries temporais indicam que a ACF perde a correlação temporal e o modelo ARIMA não consegue capturar nenhuma tendência. Além disso, o variograma espaçotemporal fica com a componente tempo próxima ao efeito pepita puro. Espera-se que a aplicação da 
deriva externa temporal local em variáveis com classe de covariância não separável obtenha resultados ainda melhores. Pois, uma vez que a tendência é removida e ainda resta a componente não separável do espaço, o variograma espaçotemporal tenderá a ser mais estruturado, com efeito pepita e variância temporal bem definidos.

A geoestatística assume a estacionariedade de segunda ordem dos dados para aquela região e garantir esta estacionariedade é fundamental para uma estimativa não enviesada. Portanto, a comparação com a geoestatística espaçotemporal (krigagem ordinária sem decomposição temporal) provou a necessidade de realizar o estudo da deriva externa temporal e a comparação com a decomposição global ilustrou a conveniência do estudo de decomposição local para melhorar a exatidão das estimativas sem suavizar os resultados, como pode ser analisado com o estudo do erro em cada pixel (Figura 41). Além disso, é notório na Figura 38 e na Figura 43 que as decomposições temporais diminuíram a variância da simulação em cada pixel simulado, ou seja conferiram mais precisão aos resultados.

Os próximos trabalhos devem seguir 2 linhas: (i) abordar variáveis espaçotemporais com variância não separável; (ii) ajuste de equações mais complexas para a decomposição local mais precisa. 


\section{REFERÊNCIAS BIBLIOGRÁFICAS}

Adamowski, K., Bocci, C., 2001, Geostatistical regional trend detection in river flow data: Hydrological Processes, v. 15, p. 3331-3341, doi:10.1002/hyp.1045.

Akaike, H., 1974, A New Look at the Statistical Model Identification: IEEE transactions on automatic control, v. 19, p. 716-723, doi: 10.1109/TAC.1974.1100705.

Armstrong, M., 1998, Basic Linear Geostatistics: Berlin, Springer - Verlag, 153p.

Assunção, R.M., Reis, I.A., Oliveira, C.D., 2001, Diffusion and prediction of Leishmaniasis in a large metropolitan area in Brazil with a Bayesian space-time model: Statist. Med., v. 20, p. 2319-2335, doi:10.1002/sim.844.

Bilonick, R.A., 1988, Monthly Hydrogen Ion Deposition Maps for The Northeastern U.S. From July 1982 to September 1984: Atmospheric Environment, v. 22, p. 1909-1924, doi: 10.1016/0004-6981(88)90080-7.

Bivand, R., Lewin-Koh, N., 2019, maptools: Tools for Reading and Handling Spatial Objects. R Package (version 0.9-5): https://cran.r-project.org/web/packages/maptools/maptools.pdf (acessado Setembro 2019).

Bivand, R.S., Pebesma, E., Gomez-Rubio, V., 2013, Applied spatial data analysis with R (2ed): New York, Springer-Verlag, 376p.

Bloomfield, P., 1976, Fourier Analysis of Time Series: an introduction: New York, John Willey \& Sons, 258p.

Box, G.E.P., Jenkins, G.M., 1970, Time Series Analysis: Forecasting and Control: San Francisco, Holden-day, 553p.

Box, G.E.P., Jenkins, G.M., Reinsel, G.C., Ljung, G.M., 2015, Time Series Analysis: Forecasting and Control (5ed): Hoboken, John Wiley and Sons, 712p.

Brockwell, P.J., Davis, R.A., 2010, Introduction to Time Series and Forecasting (2ed): New York, Springer, 437p.

Brooker, P.I., 1991, A Geostatistical Primer: Singapura, World Scientific Publishing Co Pte Ltd, 104p.

Cappello, C., De Iaco, S., Posa, D., 2018, Testing the type of non-separability and some classes of space-time covariance function models: Stochastic Environmental Research and Risk Assessment, v. 32, p. 17-35, doi: 10.1007/s00477-017-1472-2.

Carr, J.R., 1995, Numerical Analysis for the Geological Sciences: Englewood Cliffs, Prentice Hall, 592p.

Chatfield, C., 2003, The Analysis of Time Series: An Introduction: Boca Raton, CRC Press Company, 352p.

Chen, J., Boccelli, D.L., 2018, Real-time forecasting and visualization toolkit for multi-seasonal time series: Environmental Modelling \& Software, v. 105, p. 244-256, doi: 10.1016/j.envsoft.2018.03.034.

Chilès, J.P., Delfiner, P., 1999, Geostatistics: Modeling Spatial Uncertainty: New York, John Wiley \& Sons, 695p.

Clark, I., 1980, Part1: The Semivariogram, in Royle, A.G., Geostatistics: New York, McGraw Hill, p. 17-28.

Cliff, A.D., Ord, J.K., 1975, Space-Time Modelling with an Application to Regional Forecasting: Transactions of the Institute of British Geographers, v. 64, p. 119-128, doi: $10.2307 / 621469$.

Costa, A.C., Soares, A., 2009, Homogenization of Climate Data: Review and New Perspectives Using Geostatistics: Mathematical Geosciences, v. 41, p. 291-305, doi: 10.1007/s11004008-9203-3.

Cowpertwait, P.S.P., Metcalfe, A.V., 2009, Introductory Time Series with R: New York, Springer Science+Business Media, 254p. 
Cressie, N., Huang, H., 1999, Classes of Nonseparable, Spatio-temporal Stationary Covariance Functions: Journal of the American Statistical Association, v. 94, p. 1330-1340, doi: $10.2307 / 2669946$.

Cressie, N., Wikle, C.K., 2011, Statistics for Spatio-Temporal Data: Hoboken, John Wiley \& Sons, 624p.

Curceac, S., Ternynck, C., Ouarda, T.B.M.J., Chebana, F., Niang, S.D., 2019, Short-term air temperature forecasting using Nonparametric Functional Data Analysis and SARMA models: Environmental Modelling \& Software, v. 111, p. 394-408, doi: 10.1016/j.envsoft.2018.09.017.

D'Urso, P., Maharaj, E.A., Alonso, A.M., 2017, Fuzzy clustering of time series using extremes: Fuzzy Sets and Systems, v. 318, p. 56-79, doi: 10.1016/j.fss.2016.10.006.

Davis, J.C., 1986, Statistics and Data Analysis in Geology: New York, John Wiley \& Sons, $656 \mathrm{p}$.

De Cesare, L., Myers, D., Posa, D., 1997, Spatial-temporal modeling of SO2 in Milan district, in: Baafi E.Y. And Schofield N.A. (eds), Geostatistics Wollongong'96 (vol. 2): Dordrecht, Kluwer Academic Publishers, p. 1031-1042.

De Cesare, L., Myers, D.E., Posa, D., 2001a, Estimating and modeling space-time correlation structures: Statistics \& Probability Letters, v. 51, p. 9-14, doi: 10.1016/S01677152(00)00131-0.

De Cesare, L., Myers, D.E., Posa, D., 2001b, Product-sum covariance for space-time modeling: an environmental application: Environmetrics, v. 12, p. 11-23, doi: 10.1002/1099095X(200102)12:1<11::AID-ENV426>3.0.CO;2-P.

De Iaco, S., Cappello, C., Posa, D., 2018, covatest: Tests on Properties of Space-Time Covariance Functions. $\mathrm{R}$ package version 1.0.0: https://CRAN.Rproject.org/package $=$ covatest (acessado Setembro 2018).

De Iaco, S., Myers, D.E., Posa, D., 2001, Space-time analysis using a general product-sum model: Statistics \& Probability Letters, v. 52, p. 21-8, doi: 10.1016/S0167-7152(00)002005.

De Iaco, S., Myers, D.E., Posa, D., 2002, Nonseparable space-time covariance models: some parametric families: Mathematical Geology, v. 34, p. 23-42, doi: 10.1023/A:1014075310344.

De Iaco, S., Myers, D.E., Posa, D., 2003, The Linear Coregionalization Model and the ProductSum Space-Time Variogram: Mathematical Geology v. 35, p. 25-38, doi: 10.1023/A:1022425111459.

De Iaco, S., Myers, D.E., Posa, D., 2013a, Using Simultaneous Diagonalization to Identify a Space-Time Linear Coregionalization Model: Mathematical Geosciences, v. 45, p. 69-86, doi: 10.1007/s11004-012-9408-3.

De Iaco, S., Palma, M., Posa, D., 2015, Spatio-temporal geostatistical modeling for French fertility predictions: Spatial Statistics. v. 14, p. 546-562, doi: 10.1016/j.spasta.2015.10.002.

De Iaco, S., Palma, M., Posa, D., 2016, A General Procedure for selecting a class of fully symmetric space-time covariance functions: Environmetrics, v. 26, p. 212-224, doi: 10.1002/env.2392.

De Iaco, S., Posa, D., 2012, Predicting spatio-temporal random fields: Some computational aspects: Computers \& Geosciences, v. 41, p. 12-24, doi: 10.1016/j.cageo.2011.11.014.

De Iaco, S., Posa, D., Myers, D.E., 2013b, Characteristics of some classes of space-time covariance functions: Journal of Statistical Planning and Inference, v. 143, p. 2002-2015, doi: 10.1016/j.jspi.2013.06.006.

Deutsch, C.V., 2002, Geostatistical Reservoir Modeling: New York, Oxford University Press, $384 p$. 
Deutsch, C.V., Journel, A.G., 1998, GSLIB: Geostatistical Software Library and User's Guide: New York, Oxford University Press, 369p.

Dimitrakopoulos, R., Luo, X., 1994, Spatiotemporal modeling: covariances and ordinary kriging system, in Dimitrakopoulos R. (ed.), Geostatistics for the Next Century: Dordrecht, Kluwer Academic Publisher, p. 88-93.

Eidt, R.C., 1969, The Climatology of South America, in Fittkau, E.J., Illies, J., Klinge, H., Schwabe, G.H., Sioli, H., Biogeography and Ecology in South America: Dordrecht, Springer, p. 54-81.

Garcia, N.O., 1994, South American Climatology: Quaternary International, v. 21, p. 7-27, doi: 10.1016/1040-6182(94)90018-3.

Gasch, C.K., Hengl, T., Graler, B., Meyer, H., Magney, T.S., Brown, D.J., 2015, Spatiotemporal interpolation of soil water, temperature, and electrical conductivity in 3D + T: The Cook Agronomy Farm data set: Spatial Statistics, v. 14, p. 70-90, doi: 10.1016/j.spasta.2015.04.001.

Gelfand, A.E., Zhu, L., Carlin, B.P., 2001, On the change of support problem for spatiotemporal data: Biostatistics, v. 2, p. 31-45, doi: 10.1093/biostatistics/2.1.31.

Global Administrative Areas, 2018, GADM database of Global Administrative Areas (version 2.0): www.gadm.org (acessado Julho 2018).

Gneiting, T., 2002, Stationary covariance functions for space-time data. Journal of the American Statistical Association, v. 97, p. 590-600.

Gneiting, T., Genton, M.G., Guttorp, P., 2007, Geostatistical Space-Time Models, Stationarity, Separability, and Full Symmetry, in Finkenstadt, B., Held, L., Isham, V., Statistical Methods for Spatio-Temporal Systems: Boca Raton, Chapman \& Hall/CRC, p. 151-175.

Goovaerts, P., 1997, Geostatistics for National Resources Evaluation: New York, Oxford University Press, 483p.

Goovaerts, P., 2006, Geostatistical Modeling of Spaces of Local, Spatial, and Response Uncertainty For Continuous Petrophysical Properties, in Coburn, T.C., Yarus, J.M., Chamber, R.L., Stochastic Modeling and Geostatistics: Principle, Methods, and Case Studies (Volume II): Tulsa, The American Association of Petroleum Geologists (AAPG Computer Applications in Geology n ${ }^{\circ}$ 5), p. 59-79.

Gotway, C.A., Rutherford, B.M., 1994, Stochastic Simulation for Imaging Spatial Uncertainty Comparison and Evaluation of Available Algorithms, in Armstrong, M., Dowd, P.A., Geostatistical Simulations: Proceedings of the Geostatistical Simulation Workshop, Fontainebleau, France: Dordrecht, Kluwer Academic Publisher, p. 1-21.

Gräler, B., Pebesma, E., Heuvelink, G., 2016, Spatio-Temporal Interpolation using gstat: The R Journal, v. 8, p. 204-218, doi: 10.32614/RJ-2016-014.

Greenberg, J.A., Mattiuzzi, M., 2018, gdalUtils: Wrappers for the Geospatial Data Abstraction Library (GDAL) Utilities. $\mathrm{R}$ Package (version 2.0.1.14): https://cran.rproject.org/web/packages/gdalUtils/gdalUtils.pdf (acessado Setembro 2019).

GSOD - Climate Data Online (CDO), 2018, Global summary of the Day: https://www7.ncdc.noaa.gov/CDO/cdoselect.cmd?datasetabbv=GSOD\&countryabbv=\&ge oregionabbv (acessado Julho 2018).

Hannan, E.J., Quinn, B.G., 1979, The Determination of the Order of an Autoregression: Journal of the Royal Statistical Society (Series B), v. 41, p. 190-195.

Henderson-Sellers, A., McGuffie, K., 1997, Climate Models, in Thompson, R.D., Perry, A., Applied Climatology: principles and practice: New York, Routledge, p. 36-50.

Herrera, M., Ramallo-González, A.P., Eames, M., Ferreira, A.A., Coley, D.A., 2018, Creating extreme weather time series through a quantile regression ensemble: Environmental Modelling \& Software, v. 110, p. 28-37, doi: 10.1016/j.envsoft.2018.03.007. 
Hijmans, R.J., 2019, raster: Geographic Data Analysis and Modeling. R Package (version 3.02): https://cran.r-project.org/web/packages/raster/raster.pdf (acessado Setembro 2019).

Hohn, M.E., 1997, Geostatistics and Petroleum Geology: Dordrecht, Kluwer Academic Publisher, 233p.

Hristopulos, D.T., Tsantili, I.C., 2017, Space-time covariance functions based on linear response theory and the turning bands method: Spatial Statistics, v. 22, p. 321-337, doi: 10.1016/j.spasta.2017.07.001.

Hudson, G., Wackernagel, H., 1994, Mapping temperature using kriging with external drift: Theory and an example from scotlan: International Journal of Climatology, v. 14, p. 77-91, doi: 10.1002/joc.3370140107.

Hurvich, C.M., Tsai, C.L., 1989, Regression and Time Series Model Selection in Small Samples: Biometrika, v. 76, p. 297-307, doi: 10.1093/biomet/76.2.297.

Hyndman, R., Athanasopoulos, G., Bergmeir, C., Caceres, G., Chhay, L., O'Hara-Wild, M., Petropoulos, F., Razbash, S., Wang, E., Yasmeen, F., 2019, forecast: Forecasting Functions for Time Series and Linear Models. R package (version 8.9): https://CRAN.Rproject.org/package=forecast (acessado Setembro 2019).

Hyndman, R.J., Khandakar, Y., 2008, Automatic time series forecasting: the forecast package for R: Journal of Statistical Software, v. 27, p. 1-22, doi: 10.18637/jss.v027.i03.

Hyndman, R.J., Koehler, A.B., 2006. Another look at measures of forecast accuracy. $\begin{array}{llll}\text { International Journal of } & \text { Forecasting. }\end{array}$ https://doi.org/10.1016/j.ijforecast.2006.03.001

Isaaks, E.H., Srivastava, R.M., 1989, An Introduction to Applied Geostatistics: New York, Oxford University Press, $547 \mathrm{p}$.

Janacek, G., 2001, Practical Time Series: London, Hodder Education Publishers, 176p.

Jenkins, G.M., Watts, D.G., 1968, Spectral Analysis and Its Applications: San Francisco, Holden-Day, 525p.

Journel, A.G., Huijbregts, Ch.J., 1978, Mining Geostatistics: London, Academic Press, 600p.

Kamarianakis, Y., Prastacos, P., 2005, Space-time Modeling of Traffic Flow: Computers and Geoscience, v. 31, p. 119-133, doi: 10.1016/j.cageo.2004.05.012.

Kärner, O., 2009, ARIMA representation for daily solar irradiance and surface air temperature time series: Journal of Atmospheric and Solar-Terrestrial Physics, v. 71, p. 841-847, doi: 10.1016/j.jastp.2009.03.018.

Kedem, B., Fokianos, K., 2002, Regression Models for Time Series Analysis: Hoboken, John Wiley \& Sons, 360p.

Kilibarda, M., Hengl, T., Heuvelink, G.B.M., Graler, B., Pebesma, E., Tadić, M.P, Bajat, B., 2014, Spatio-temporal interpolation of daily temperatures for global land areas at $1 \mathrm{~km}$ resolution: Journal of Geophysical Research: Atmospheres, v. 119, p. 2294-2313, doi: 10.1002/2013JD020803.

Kilibarda, M., Tadić, M.P., Hengl, T., Luković, J., Bajat, B., 2015, Global geographic and feature space coverage of temperature data in the context of spatio-temporal interpolation: Spatial Statistics, v. 14, p. 22-38, doi: 10.1016/j.spasta.2015.04.005.

Kitanidis, P.K., 1997, Introduction to Geostatistics: Application in Hydrogeology: Cambridge, Press Syndicate of the University of Cambridge, 249p.

Koutroumanidis, T., Iliadis, L., Sylaios, G.K., 2006, Time-series modeling of fishery landings using ARIMA models and Fuzzy Expected Intervals software: Environmental Modelling \& Software, v. 21, p. 1711-1721, doi: 10.1016/j.envsoft.2005.09.001.

Kyriakidis, P., Journel A.G., 1999, Geostatistical Space-Time Models: A Review: Mathematical Geology, v. 31, p. 651-684, doi: 10.1023/A:1007528426688.

Landim, P.M.B., 1997, Análise estatística de dados geológicos: São Paulo, Editora UNESP, p. $156-158$. 
Lantuejoul, C., 2002, Geostatistical Simulation: Models and Algorithms: Berlin, Springer, $256 \mathrm{p}$.

Lau, W., 2006, ESSAY: Model interpretation of climate signals: An application to Asian monsoon climate, in Bridgman H., Oliver J., The Global Climate System: Patterns, Processes, and Teleconnections: Cambridge, Cambridge University Press, p. 281-308, doi: 10.1017/CBO9780511817984.010.

Li, B., Genton, M.G., Sherman, M., 2007, A Nonparametric Assessment of Properties of SpaceTime Covariance Functions: Journal of the American Statistical Association, v. 102, p. 736744, doi: 10.1198/016214507000000202.

Li, G., Haining, R., Richardson, S., Best, N., 2014, Space-time variability in burglary risk: A Bayesian spatio-temporal modelling approach: Spatial Statistics, v. 9, p. 180-191, doi: 10.1016/j.spasta.2014.03.006.

Liu, C., Koike, K., 2007, Extending Multivariate Space-Time Geostatistics for Environmental Data Analysis: Mathematical Geology, v. 39, p. 289-305, doi: 10.1007/s11004-007-90859.

Ma, C., 2002, Spatio-temporal covariance functions generated by mixtures: Mathematical Geology, v. 34, p. 965-75, doi: 10.1023/A:1021368723926.

Ma, C., 2003a, Spatio-temporal stationary covariance models: Journal of Multivariate Analysis, v. 86, p. 97-107, doi: 10.1016/S0047-259X(02)00014-3.

Ma, C., 2003b, Nonstationary covariance functions that model space-time interactions: Statistics \& Probability Letters, v. 61, p. 411-419, doi: 10.1016/S0167-7152(02)00401-7.

Maçaira, P.M., Thomé, A.M.T., Oliveira, F.L.C., Ferrer, A.L.C., 2018, Time series analysis with explanatory variables: A systematic literature review: Environmental Modelling \& Software, v. 107, p. 199-209, doi: 10.1016/j.envsoft.2018.06.004.

Manzione, R.L., Takafuji, E.H.M., De Iaco, S., Cappello, C., Rocha, M.M., 2018, Exploring the spatio-temporal nature of shallow groundwater monitoring data using geostatistical approach, Groundwater and life: Science and Technology into Action, Daejeon, 45th International Association of Hydrogeologists Congress, http://www.iah2018.org.

Manzione, R.L., Takafuji, E.H.M., De Iaco, S., Cappello, C., Rocha, M.M., 2019, Spatiotemporal Kriging to Predict Water Table Depths from Monitoring Data in a Conservation Area at São Paulo State, Brazil: Geoinformatics \& Geostatistics: An Overview, v. 7, doi: 10.4172/2327-4581.1000205.

Manzione, R.L., Takafuji, E.H.M., Rocha, M.M., 2016, Modelling spatio-temporal variability of water table depths monitoring data, Groundwater and society: 60 years of IAH, Montpellier, 43rd IAH International Congress.

Manzione, R.L., Takafuji, E.H.M., Rocha, M.M., 2017, Spatio-temporal interpolation of water table depths in a conservation area at Bauru Aquifer System (SP/Brazil), Groundwater: heritage and sustainability, Dubrovinik, 44th IAH International Congress.

Martínez, W.A., Melo, C.E., Melo, O.O., 2017, Median Polish Kriging for space-time analysis of precipitation: Spatial Statistics, v. 19, p. 1-20, doi: 10.1016/j.spasta.2016.10.003.

Matheron, G., 1963, Principles of geostatistics: Economic Geology, v. 58, p. 1246-1266, doi: 10.2113/gsecongeo.58.8.1246.

Matheron, G., 1971, The Theory of Regionalized Variables and its Applications: École Nationale Supérieure des Mines de Paris, Fontainebleau, Les Cahiers du Centre de Morphologie Mathématique de Fontainebleau.

Menezes, R., Piairo, H., Garcia-Soidán P., Sousa I., 2016, Spatial-temporal modellization of the NO2concentration data through geostatistical tools: Statistical Methods \& Applications, v. 25, p. 107-124, doi: 10.1007/s10260-015-0346-3. 
Monteiro, A., Menezes, R., Silva M.E., 2017, Modelling spatio-temporal data with multiple seasonalities: The NO2 Portuguese case: Spatial Statistics, v.22, p. 371-387, doi: 10.1016/j.spasta.2017.04.005.

Montero, J.-M., Fernández-Avilés, G., Mateu, J., 2015, Spatial and Spatio-Temporal Geostatistical Modeling and Kriging: Chichester, John Wiley \& Sons, Ltd, 400p.

Montgomery, D.C., Jennings, C.L., Kulahci, M., 2015, Introduction to Time Series Analysis and Forecasting (2ed): Hoboken, John Wiley \& Sons, 472p.

Myers, D.E., 2006, Reflections on Geostatistics and Stochastic Modeling, in Coburn, T.C., Yarus, J.M., Chamber, R.L., Stochastic Modeling and Geostatistics: Principle, Methods, and Case Studies (Volume II): Tulsa, The American Association of Petroleum Geologists (AAPG Computer Applications in Geology n ${ }^{\circ}$ ), p. 11-22.

Nash, E., James, P., Parker, D., 2005, A Model for spatio-temporal network planning: Computers and Geoscience, v. 31, p. 135-143, doi: 10.1016/j.cageo.2004.06.012.

Newbold, P., Agiakloglou, C., Miller, J., 1993, Long-term inference based on short-term forecasting models, in Rao, T.S., Developments in Time Series Analysis: London, Chapman \& Hall, p 9-25.

Nunes, C., Soares, A., 2005, Geostatistical space-time simulation model for air quality prediction: Envirometrics, v. 16, p. 393-404, doi: 10.1002/env.710.

Olea, R.A., 1994, Fundamental of Semivariogram Estimation, Modeling and Usage, in Yarus, J.M., Chamber, R.L., Stochastic Modeling and Geostatistics: Principle, Methods, and Case Studies: Tulsa, The American Association of Petroleum Geologists (AAPG Computer Applications in Geology n'3), p. 27-35.

Olea, R.A., 1999, Geostatistics for Engineers and Earth Scientists: New York, Springer, 303p.

Paci, L., Beamonte, M.A., Gelfand, A.E., Gargallo, P., Salvador, M., 2017, Analysis of residential property sales using space-time point patterns: Spatial Statistics, v. 21, p. 149165, doi: 10.1016/j.spasta.2017.06.007.

Paci, L., Gelfand, A.E., Holland, D.M., 2013, Spatio-temporal modeling for real-time ozone forecasting: Spatial Statistics, v. 4, p.79-93, doi: 10.1016/j.spasta.2013.04.003.

Pebesma, E., 2012, spacetime: Spatio-Temporal Data in R: Journal of Statistical Software, v. 51, p. 1-30, doi: 10.18637/jss.v051.i07.

Pebesma, E., 2018, spacetime: Classes and Methods for Spatio-Temporal Data. R Package (version 1.2-2): https://cran.r-project.org/web/packages/spacetime/spacetime.pdf (acessado Setembro 2019).

Pebesma, E., Graeler, B., 2019, gstat: Spatial and Spatio-Temporal Geostatistical Modelling, Prediction and Simulation. R Package (version 2.0-2): https://cran.rproject.org/web/packages/gstat/gstat.pdf (acessado Setembro 2019).

Pebesma, E.J., 2004, Multivariable geostatistics in S: the gstat package: Computers \& Geosciences, v. 30, p. 683-691, doi: 10.1016/j.cageo.2004.03.012.

Pfeifer, P.E., Deutsch, S.J., 1980, A STARIMA Model-Building Procedure with Application to Description and Regional Forecasting: Transactions of the Institute of British Geographers, v. 5, p. 330-349, doi: 10.2307/621846.

Piroutek, A., Assunção, R., Paiva, T., 2014, Space-time prospective surveillance based on Knox local statistics: Statist. Med.,v. 33, p. 2758-2773, doi:10.1002/sim.6118.

Poggio, L., Gimona, A., 2015, Downscaling and correction of regional climate models outputs with a hybrid geostatistical approach: Spatial Statistics, v. 14, p. 4-21, doi: 10.1016/j.spasta.2015.04.006.

Porcu, E., Gregori, P., Mateu, J., 2006, Nonseparable stationary anisotropic space-time covariance functions: Stochastic Environmental Research and Risk Assessment, v. 21, p. 113-122, doi: 10.1007/s00477-006-0048-3. 
Porcu, E., Mateu, J., 2007, Mixture-based modeling for space-time data: Environmetrics, v. 18, p. 285-302, doi: 10.1002/env.832.

Porcu, E., Mateu, J., Christakos, G., 2009, Quasi-arithmetic means of covariance functions with potential applications to space-time data: Journal of Multivariate Analysis, v. 100, p. 1830 1844, doi: 10.1016/j.jmva.2009.02.013.

Prado, R., West, M., 2010, Time Series: Modeling, Computation, and Inference: Boca Raton, CRC Press, 368p.

Prates, M.O., Kulldorff, M., Assunção, R.M., 2014, Relative risk estimates from spatial and space-time scan statistics: are they biased?: Statist. Med., v. 33, p. 2634-2644, doi:10.1002/sim.6143.

Pyrcz, M.J., Griegarten, R., Frykman, P., Deutsch, C.V., 2006, Representative Input Parameters for Geostatistical Simulations, in Coburn, T.C., Yarus, J.M., Chamber, R.L., Stochastic Modeling and Geostatistics: Principle, Methods, and Case Studies (Volume II): Tulsa, The American Association of Petroleum Geologists (AAPG Computer Applications in Geology no5), p. 123-137.

R Core Team, 2017, R: A language and environment for statistical computing. R Foundation for Statistical Computing, Vienna, Austria: https://www.R-project.org/

Reisen, V.A., Sarnaglia, A.J.Q., Reis Jr, N.C., Lévy-Leduc, C., Santos, J.M., 2014, Modeling and forecasting daily average PM10 concentrations by a seasonal long-memory model with volatility: Environmental Modelling \& Software, v. 51, p. 286-295, doi: 10.1016/j.envsoft.2013.09.027.

Ribeiro Jr, P.J., Diggle, P.J., 2018, geoR: Analysis of Geostatistical Data. R Package (version 1.7-5.2.1): https://cran.r-project.org/web/packages/geoR/geoR.pdf (acessado Setembro 2019).

Rouhani, S., Hall, T.J., 1989, Space-time kriging of groundwater data, in Armstrong, M., Geostatistics: Dordrecht, Kluwer Academic Publishers, p. 639-51.

Russo, A., Soares, A.O., 2014, Hybrid Model for Urban Air Pollution Forecasting: A Stochastic Spatio-Temporal Approach: Mathematical Geosciences, v. 46, p. 75-93, doi: 10.1007/s11004-013-9483-0.

Scheffel, J., Lindvall, K., Yik, H.F., 2018, A time-spectral approach to numerical weather prediction: Computer Physics Communications, v. 226, p. 127-135, doi: 10.1016/j.cpc.2018.01.010.

Schmertmann, C.P., Assunção, R.M., Potter, J.E., 2010, Knox meets Cox: Adapting epidemiological space-time statistics to demographic studies: Demography, v. 47, p. 629650, doi: 10.1353/dem.0.0113.

Schwarz, G., 1978, Estimating the dimension of a model: The Annals of Statistics, v. 6, p. 461 464.

Sherman, M., 2011, Spatial Statistics and Spatio-Temporal Data: Covariance Functions and Directional Properties: Chichester, John Wiley \& Sons, 294p.

Shumway, R.H., Stoffer, D.S., 2011, Time series analysis and its application: with R examples (3ed): New York, Springer Science+Business Media, 576p.

Snepvangers, J.J.J.C., Heuvelink, G.B.M., Huisman, J.A., 2003, Soil water content interpolation using spatio-temporal kriging with external drift: Geoderma, v. 112, p. 253 271, doi: 10.1016/S0016-7061(02)00310-5.

Soares, A., Pereira, M.J., 2007, Space-time modelling of air quality for environmental-risk maps: A case study in South Portugal: Computers and Geosciences, v. 33, p. 1327-1336, doi: 10.1016/j.cageo.2007.06.001.

Sølna, K., Switzer, P., 1996, Time Trend Estimation for a Geographic Region: Journal of the American Statistical Association, v. 91, p. 577-589, doi: 10.1080/01621459.1996.10476927. 
Srivastava, R.M., 1994, An Overview of Stochastic Methods for Reservoir Characterization, in Yarus, J.M., Chamber, R.L., Stochastic Modeling and Geostatistics: Principle, Methods, and Case Studies: Tulsa, The American Association of Petroleum Geologists (AAPG Computer Applications in Geology n³,), p. 3-6.

Stein, M.L., 2005, Space-time covariance functions: Journal of the American Statistical Association, v. 100, p. 310-21, doi: 10.1198/016214504000000854.

Stull, R.B., 2000, Meteorology for Scientists and Engineers (2nd ed): Pacific Grove, Brooks/Cole, 528p.

Takafuji, E.H.M., Basic, B.L.J., Siqueira-Gay, J., Giannotti, M.A., Rocha, M.M., 2017, Geostatistics as a Tool to Map the Spatio-Temporal Evolution of Car Ownership in São Paulo Metropolitan Area, in Proceedings, XVIII Simpósio Brasileiro de Sensoriamento Remoto, Santos, Simpósio Brasileiro de Sensoriamento Remoto, p. 6147-6154.

Takafuji, E.H.M., Rocha, M.M., Manzione, R.L., 2016, Forecasting groundwater levels and assessing its uncertainty using SGS and ARIMA - a case study in the Bauru aquifer system - Brazil, in Proceedings, Book of Abstracts: Geostatistics for Environmental Applications geoENV 2016, Lisboa, geoENV - GEOSTATISTICS FOR ENVIRONMENTAL APPLICATIONS, p. 87-93.

Takafuji, E.H.M., Rocha, M.M., Manzione, R.L., 2018, Groundwater Level Prediction/Forecasting and Assessment of Uncertainty Using SGS and ARIMA Models: A Case Study in the Bauru Aquifer System (Brazil): Natural Resources Research, v. 28, p. 487-503, doi: 10.1007/s11053-018-9403-6.

Takafuji, E.H.M., Rocha, M.M., Manzione, R.L., 2020, Spatiotemporal forecast with local temporal drift applied to weather patterns in Patagonia: Springer Nature Applied Sciences, v. 2 doi: 10.1007/s42452-020-2814-0.

Tamayo-Uria, I., Mateu, J., Diggle, P.J., 2014, Modelling of the spatio-temporal distribution of rat sightings in an urban environment: Spatial Statistics, v. 9, p. 192-206, doi: 10.1016/j.spasta.2014.03.005.

Tsay, R.S., 2010, Analysis of Financial Time Series (3rd ed): Hoboken, John Wiley \& Sons, 720 p.

Wan, Z., Hook, S., Hulley, G., 2015, MOD11C3 MODIS/Terra Land Surface Temperature/Emissivity Monthly L3 Global 0.05Deg CMG V006. NASA EOSDIS Land Processes DAAC: https://doi.org/10.5067/MODIS/MOD11C3.006 (acessado Fevereiro 2018).

Wang, H., Pardo-Igúzquiza, E., Dowd, P.A., Yang, Y., 2017, Optimal estimation of areal values of near-land-surface temperatures for testing global and local spatio-temporal trends: Computers \& Geosciences, v. 106, p. 109-117, doi: 10.1016/j.cageo.2017.06.002.

Watanabe, J., 2003, Implementação do algoritmo de simulação estocástica pelo método de bandas rotativas em Delphi [Monografia de Trabalho de Formatura (TF-2003/34)]: São Paulo, Instituto de Geociências - Universidade de São Paulo.

Woodward, W.A., Gray, H.L., Elliott, A.C., 2011, Applied Time Series Analysis: Boca Raton, CRC Press, 564p.

$\mathrm{Xu}$, Y., Shen, Y., 2013, Reconstruction of the Land Surface Temperature time series using harmonic analysis: Computers \& Geosciences, v. 61, p. 126-132, doi: 10.1016/j.cageo.2013.08.009.

Yamamoto, J.K., 2001a, Análise Geoestatística, in Yamamoto, J.K., Avaliação e Classificação de Reservas Minerais: São Paulo, Edusp, p. 69-91.

Yamamoto, J.K., 2001b, Métodos Computacionais, in Yamamoto, J.K., Avaliação e Classificação de Reservas Minerais: São Paulo, Edusp, p. 123-161.

Yoo, C., Im, J., Park, S., Quackenbush, L.J., 2018, Estimation of daily maximum and minimum air temperatures in urban landscapes using MODIS time series satellite data: ISPRS Journal 
of Photogrammetry and Remote Sensing, v. 137, p. 149:162, doi: 10.1016/j.isprsjprs.2018.01.018.

Zeileis, A., Grothendieck, G., 2005, zoo: S3 Infrastructure for Regular and Irregular Time Series: Journal of Statistical Software, v. 14, p. 1-27, doi: 10.18637/jss.v014.i06.

Zeileis, A., Grothendieck, G., Ryan, J.A., Ulrich, J.M., Andrews, F., 2019, zoo: S3 Infrastructure for Regular and Irregular Time Series (Z's Ordered Observations). R Package (version 1.8-6): https://cran.r-project.org/web/packages/zoo/zoo.pdf (acessado Setembro 2019).

Zhong, X., Kealy, A., Duckham, M., 2016, Stream Kriging: Incremental and recursive ordinary Kriging over spatiotemporal data streams: Computers and Geosciences, v. 90, p. 134-143, doi: 10.1016/j.cageo.2016.03.004.

Zhou, J., Jia, 1., Menenti, M., 2015, Reconstruction of global MODIS NDVI time series: Performance of Harmonic Analysis of Time Series (HANTS): Remote Sensing of Environment, v. 163, p. 217-228, doi: 10.1016/j.rse.2015.03.018. 